\title{
Copyright
}

by

William Lloyd Bircher

2010 
Dissertation Committee for William Lloyd Bircher

certifies that this is the approved version of the following dissertation:

\section{Predictive Power Management for Multi-Core Processors}

Committee:

Lizy John, Supervisor

Mattan Erez

Steve Keckler

Charles Lefurgy

Tess Moon

David Pan 


\title{
Predictive Power Management for Multi-Core Processors
}

\author{
by
}

William Lloyd Bircher, B.S.E.E.; M.S.E.

\section{Dissertation}

Presented to the Faculty of the Graduate School of

The University of Texas at Austin

in Partial Fulfillment

of the Requirements

for the Degree of

Doctor of Philosophy

The University of Texas at Austin

December 2010 
To Sara, Catherine and Elizabeth 


\section{Acknowledgements}

I would like to thank Dr Lizy John for her guidance, insight and flexibility. Her positive attitude and encouragement were instrumental in staying motivated. I appreciate her balanced approach to work, study and life that has made my doctoral study possible.

I am grateful to my committee members, Prof. Mattan Erez, Prof. Steve Keckler, Dr. Charles Lefurgy, Prof. Tess Moon and Prof. David Pan. Your helpful comments and suggestions greatly improved the quality of the dissertation.

I am thankful to my fellow researchers in the Laboratory for Computer Architecture (LCA) for our collaborations, discussions and practice talks over the years. I enjoyed working with Dr. Madhavi Valluri and Jason Law on power modeling. This collaboration was a great introduction to graduate research and proved to be a strong basis for my thesis. I look forward to future collaboration with Karthik Ganesan, Jungho Jo and the other authors who worked on the synthetic power virus paper. I would like to thank Dimitris Kaseridis and Ciji Isen for many great discussions on research and life. I am grateful to the other members of LCA, Ajay Joshi, Aashish Phansalkar, Juan Rubio, Ravi Bhargava, Byeong Lee, Tao Li, Jeff Stuecheli, Jian Chen, Arun Nair, Muhammad Farooq and Jungho Jo for attending my practice talks and providing insightful feedback.

I am thankful to Tommy Tam, Gwen Bernstrom and Dr. Charles Lefurgy at International Business Machines for encouraging me to pursue graduate education. 
Thanks to Brent Kelly at Advanced Micro Devices for convincing me to join his power and performance modeling team while completing my $\mathrm{PhD}$. Working on his team has allowed me to "amortize" the considerable efforts required for doctoral research with gainful employment.

Finally, I would like to thank my dear wife Sara. Doctoral study requires a major commitment from family and friends. Doctoral study while working full-time, raising a family and remodeling a home requires an incredible commitment. I am eternally grateful for your unwavering encouragement, support and patience without which this dissertation would not have been possible. 


\title{
Predictive Power Management for Multi-Core Processors
}

\author{
William Lloyd Bircher, PhD. \\ The University of Texas at Austin, 2010
}

Supervisor: Lizy John

Energy consumption by computing systems is rapidly increasing due to the growth of data centers and pervasive computing. In 2006 data center energy usage in the United States reached 61 billion kilowatt-hours (KWh) at an annual cost of 4.5 billion USD [P108]. It is projected to reach 100 billion KWh by 2011 at a cost of 7.4 billion USD. The nature of energy usage in these systems provides an opportunity to reduce consumption.

Specifically, the power and performance demand of computing systems vary widely in time and across workloads. This has led to the design of dynamically adaptive or power managed systems. At runtime, these systems can be reconfigured to provide optimal performance and power capacity to match workload demand. This causes the system to frequently be over or under provisioned. Similarly, the power demand of the system is difficult to account for. The aggregate power consumption of a system is composed of many heterogeneous systems, each with a unique power consumption characteristic.

This research addresses the problem of when to apply dynamic power management in multi-core processors by accounting for and predicting power and performance demand 
at the core-level. By tracking performance events at the processor core or thread-level, power consumption can be accounted for at each of the major components of the computing system through empirical, power models. This also provides accounting for individual components within a shared resource such as a power plane or top-level cache. This view of the system exposes the fundamental performance and power phase behavior, thus making prediction possible.

This dissertation also presents an extensive analysis of complete system power accounting for systems and workloads ranging from servers to desktops and laptops. The analysis leads to the development of a simple, effective prediction scheme for controlling power adaptations. The proposed Periodic Power Phase Predictor (PPPP) identifies patterns of activity in multi-core systems and predicts transitions between activity levels. This predictor is shown to increase performance and reduce power consumption compared to reactive, commercial power management schemes by achieving higher average frequency in active phases and lower average frequency in idle phases. 


\section{Table of Contents}

\section{Chapter 1 Introduction ..........................................................................................................................1}

1.1 Attributing Power in Multi-Core Systems .................................................... 1

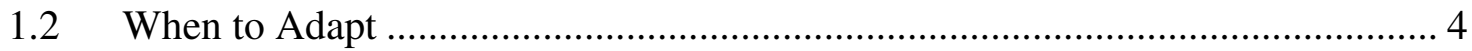

1.3 Power Variation is Periodic........................................................................... 6

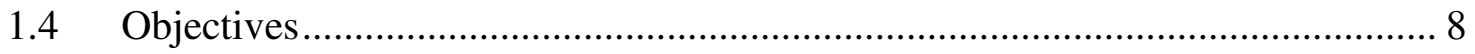

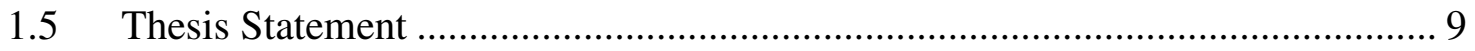

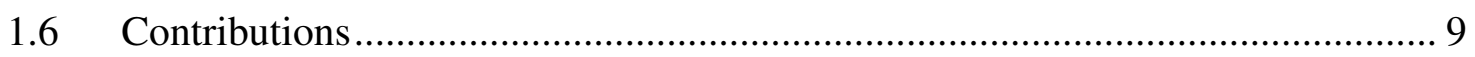

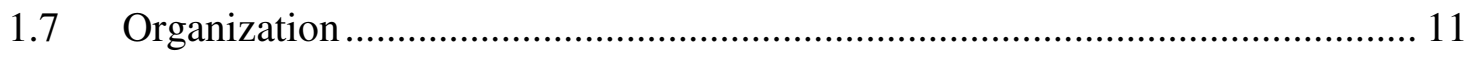

Chapter 2 Methodology .........................................................................................13

2.1 Measuring System and Component Power ............................................... 13

2.1.1 Aggregate CPU Power Measurement ..................................................... 14

2.1.2 Subsystem-Level Power in a Server System ........................................ 15

2.1.3 Subsystem-Level Power in a Mobile System ......................................... 17

2.2 Controlling Temperature, Voltage and Frequency........................................ 19

2.3 Performance Counter Sampling .......................................................... 21

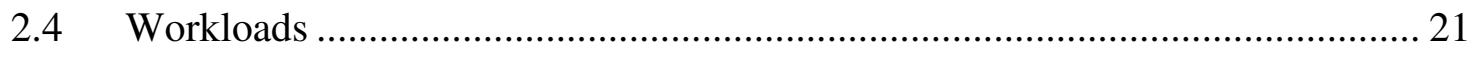

Chapter 3 Modeling CPU Power using Performance Monitoring Counters .............24

3.1 Correlation of Performance Counters to Power ............................................... 24

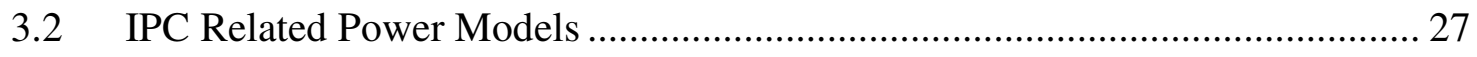

3.3 Micro ROM Related Power Models.............................................................. 30

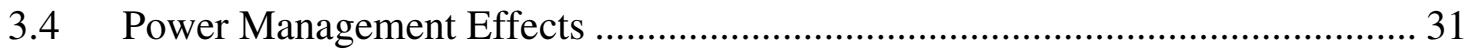

3.4.1 Active and Idle Power Management ...................................................... 32

3.4.2 Active Power Management: P-states ..................................................... 33

3.4.3 Idle Power Management: C-states ......................................................... 34

3.4.4 Case Study: Processor Power Management Characteristics ...................... 34 
3.4.5 Power Management-Aware Model ........................................................ 37

3.5 Methodology for Power Modeling ............................................................ 40

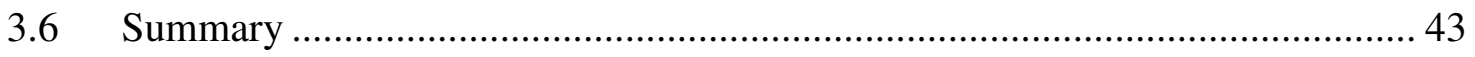

Chapter 4 System-Level Power Analysis ..............................................................44

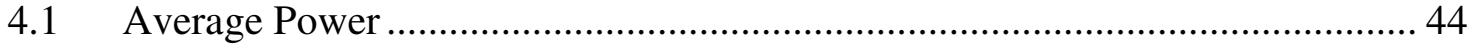

4.1.1 Server Platform - SPEC CPU, SPECjbb and DBT-2 .............................. 44

4.1.2 SPEC CPU 2000/2006 CPU and Memory Power Comparison .................. 46

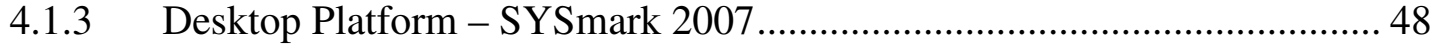

4.1.4 Desktop Platform - SPEC CPU, 3DMark and SYSmark.......................... 49

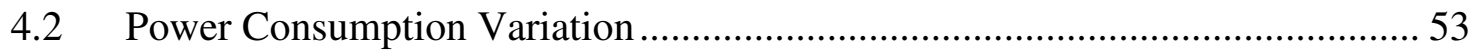

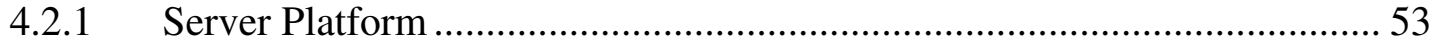

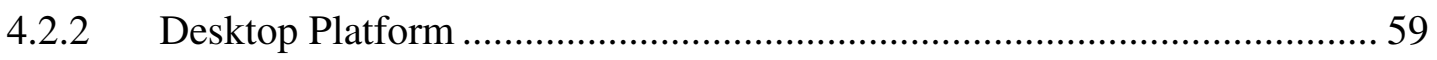

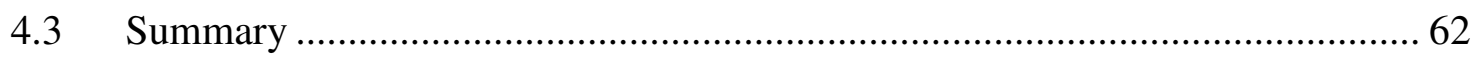

Chapter 5 Modeling System-Level Power using Trickle-Down Events......................64

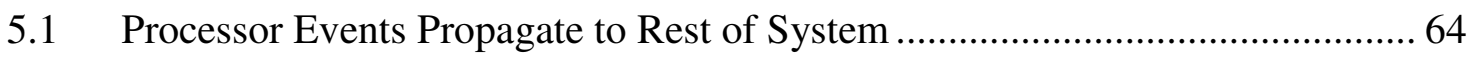

5.2 Complete-System Server Power Model ....................................................... 67

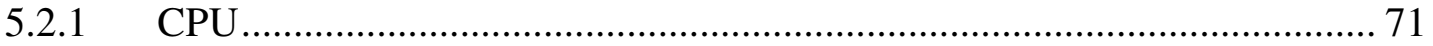

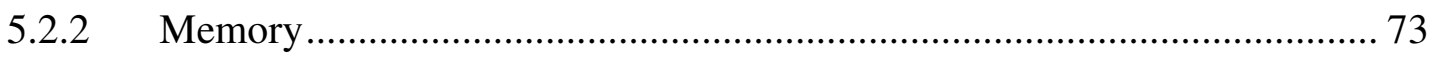

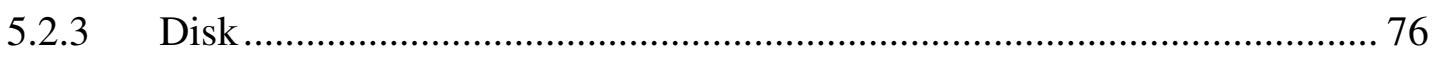

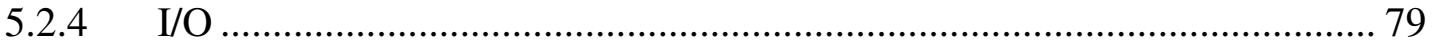

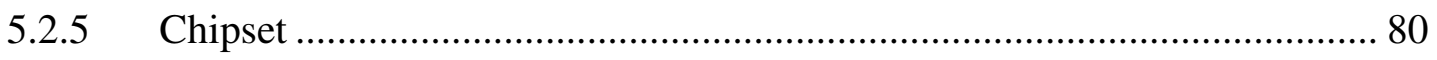

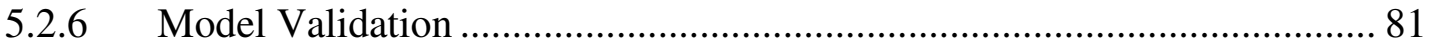

5.3 Complete-System Desktop Power Model .................................................. 84

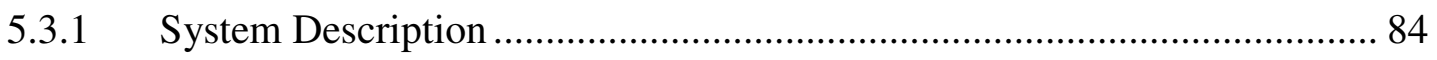

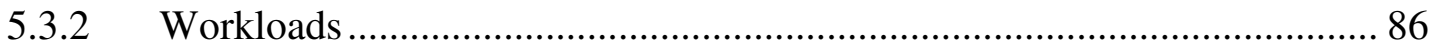

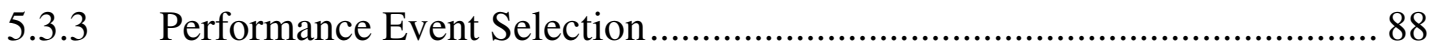

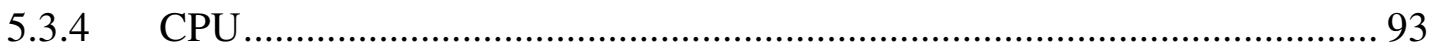

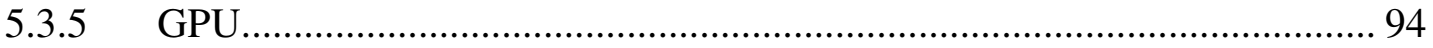




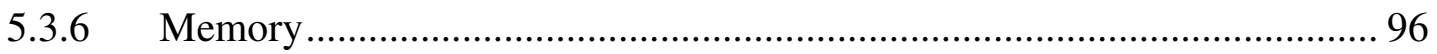

5.3.7 Memory Controller ........................................................................... 98

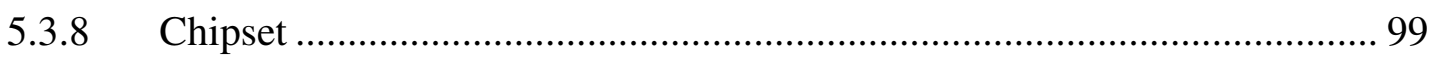

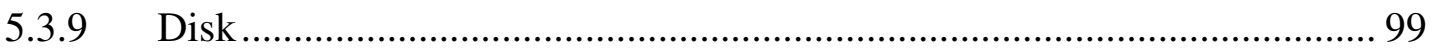

5.3.10 Model Validation .................................................................................... 99

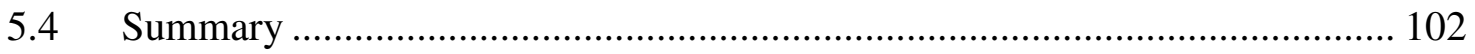

Chapter 6 Performance Effects of Dynamic Power Management ...............................103

6.1 Direct and Indirect Performance Impacts.......................................................... 103

6.1.1 Transition Costs .................................................................................. 103

6.1.2 Workload Phase and Policy Costs ............................................................ 104

6.1.3 Performance Effects ............................................................................ 105

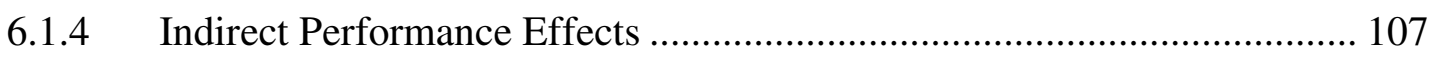

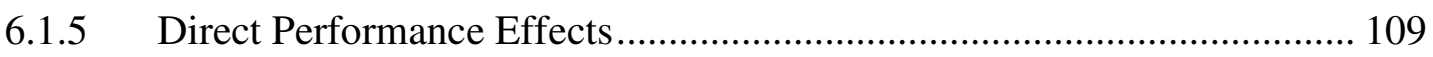

6.2 Reactive Power Management ..................................................................... 110

6.2.1 Power and Performance Results ......................................................... 116

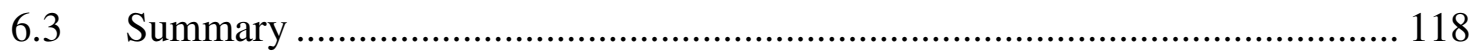

Chapter 7 Predictive Power Management...............................................................................120

7.1 Core-Level Activity Prediction ……………............................................. 120

7.2 Commercial DVFS Algorithm ................................................................ 125

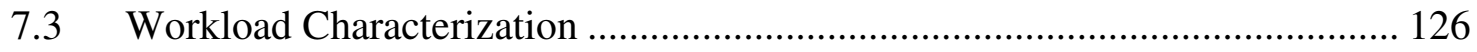

7.4 Periodic Power Phase Predictor - PPPP ...................................................... 130

7.5 Predicting Core Activity Level ..................................................................... 134

7.6 Predicting Power Levels............................................................................... 143

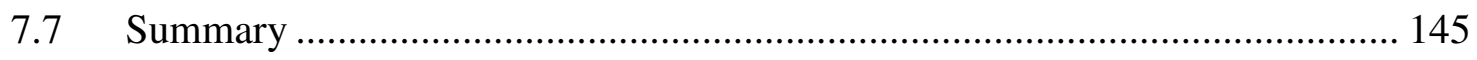

Chapter 8 Related Research .............................................................................................147

8.1 Performance Counter-Driven Power Models ...................................................... 147

8.2 System-Level Power Characterization ................................................................ 149

8.3 Predictive Power Adaptation.......................................................................... 150 
8.4 Deadline and User-Driven Power Adaptation................................................. 151

Chapter 9 Conclusions and Future Work ...........................................................153

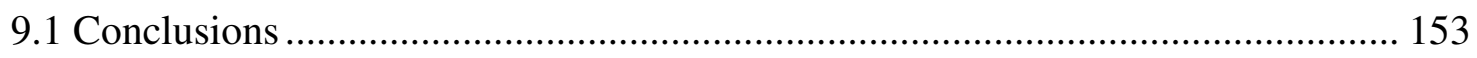

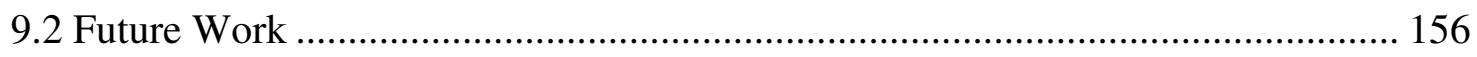

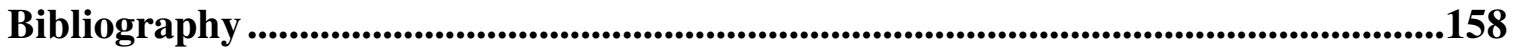

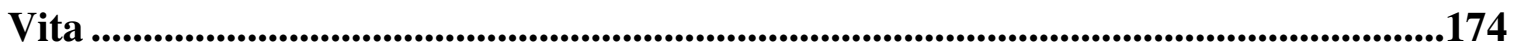




\section{List of Tables}

Table 1.1 Windows Vista Reactive DVFS ............................................................... 5

Table 2.1 Desktop System Description .................................................................. 14

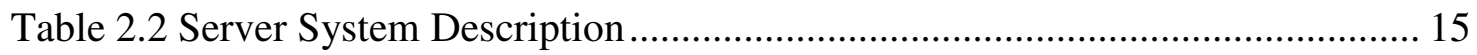

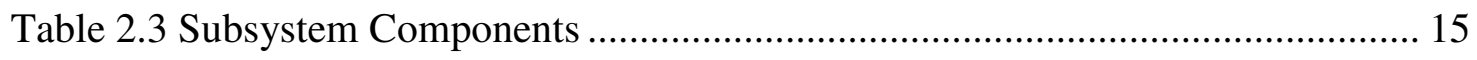

Table 2.4 Laptop System Description .................................................................. 19

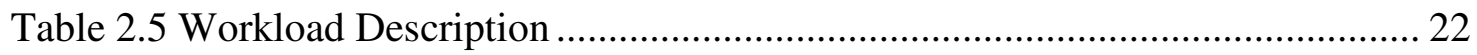

Table 3.1. Intel Pentium 4, High and Low Correlation Performance Metrics ............. 25

Table 3.2 Percent of Fetched $\mu$ ops Completed/Retired - SPEC CPU 2000 ............... 26

Table 3.3 нop Linear Regression Model Comparison ........................................... 28

Table 3.5 Instruction Power Consumption......................................................... 30

Table 3.6 нop Linear Regression Model Comparison .............................................. 31

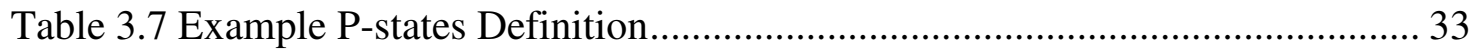

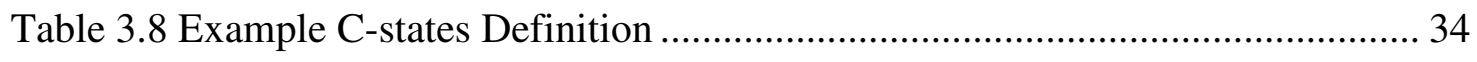

Table 3.9 AMD Quad-Core Power Model ................................................................... 39

Table 4.1 Subsystem Power Standard Deviation (Watts) ........................................ 53

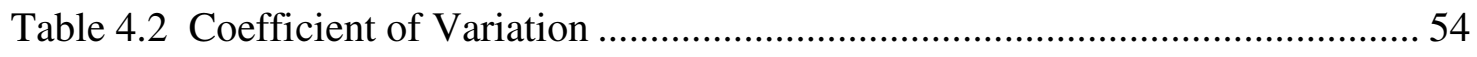

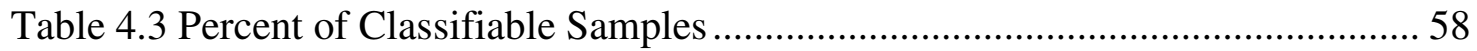

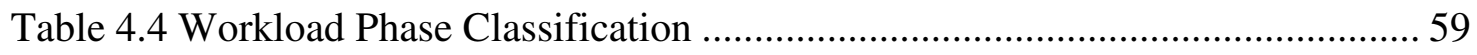

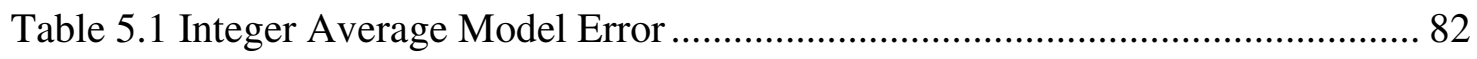

Table 5.2 Floating Point Average Model Error.......................................................... 83

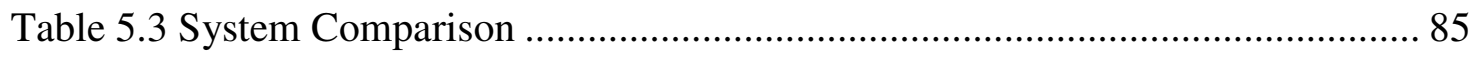

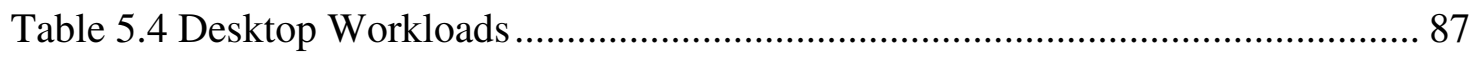

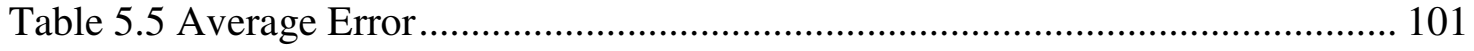

Table 6.1 Performance Loss Due to Low Idle Core Frequency - SPEC CPU 2006 .. 111

Table 6.2 Power/Performance Study: SYSmark 2007 .......................................... 116 


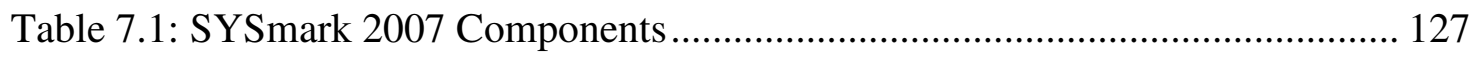

Table 7.2: Periodic Power Phase Predictor Field Descriptions................................. 134

Table 7.3: SYSmark 2007 DVFS Hit Rate ........................................................... 136

Table 7.4: SYSmark 2007 Prediction Coverage ................................................... 137

Table 7.5: Core Phase Residency by Length....................................................... 138

Table 7.6: SYSmark 2007 Power and Performance Impact of PPPP ....................... 141

Table 7.7: SYSmark 2007 P-State and C-State Residency of PPPP versus Reactive. 142 


\section{List of Figures}

Figure 1.1 CPU Core-Level Power Accounting.......................................................... 3

Figure 1.2 System-Level Power Accounting ..................................................... 4

Figure 1.3 Core Activity Patterns - Blu-Ray Playback ............................................ 7

Figure 1.4 Thread and Aggregate Power Patterns....................................................... 8

Figure 2.1 Current Sense Amplification PCB ..................................................... 16

Figure 2.2 Power Measurement Environment ........................................................ 17

Figure 2.3 Leakage Power Determination............................................................... 20

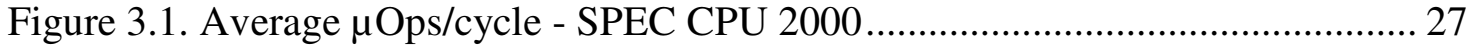

Figure 3.2 Temperature Sensitivity of Leakage Power............................................. 35

Figure 3.3 Power by C-state/P-state Combination .................................................. 36

Figure 3.4 CPU Power Model - SPEC CPU 2006 Power and Average Error............. 39

Figure 3.5 Trickle-Down Modeling Process ............................................................ 42

Figure 4.1 Average Power Consumption (Watts) …......................................... 45

Figure 4.2 CPU2000 Average Core Power - 1 Thread vs. 4 Thread........................... 47

Figure 4.3 CPU2006 Average Core Power - 1 Thread vs. 4 Thread........................... 47

Figure 4.4 SPEC CPU2006 Average Core vs. DIMM Power.................................... 48

Figure 4.5 Desktop Subsystem Power Breakdown ................................................. 49

Figure 4.6 Subsystem Average Power (Watts) ....................................................... 52

Figure 4.7 Subsystem Amplitude Distributions .................................................. 57

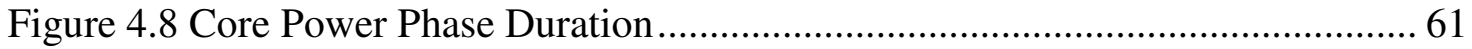

Figure 4.9 Core Power Phases - SYSmark 2007 ............................................... 62

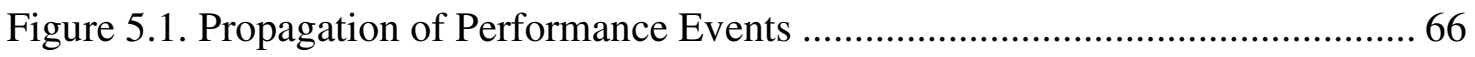

Figure 5.2 Processor Power Model - gcc................................................................ 72

Figure 5.3 Memory Power Model (L3 Misses) - mesa.......................................... 74

Figure 5.4 Prefetch and Non-Prefetch Bus Transactions - mcf .............................. 75 
Figure 5.5 Memory Power Model (Memory Bus Transactions)- mcf ........................ 76

Figure 5.6 Disk Power Model (DMA+Interrupt) - Synthetic Disk Workload ............ 79

Figure 5.7 GPU Power Model (Non-Gated Clocks) - 3DMark06-HDR1 ................... 96

Figure 5.8 DRAM Power Model (¿DCT Access, LinkActive) - SYSmark 2007-3D. 97

Figure 5.9 Memory Controller Power ( $\sum$ DCT Access, LinkActive) - HDR1 ............ 98

Figure 6.1 Direct and Indirect Performance Impact............................................ 107

Figure 6.2 Remote and Local Probe Sensitivity ................................................. 112

Figure 6.3 C-state vs. P-state Performance ................................................................ 113

Figure 6.4 Varying OS P-state Transition Rates .................................................. 114

Figure 7.1: Thread and Aggregate Power Patterns ............................................... 123

Figure 7.2: Windows Vista Reactive P-State Selection Algorithm ......................... 126

Figure 7.3: Six-Core Phenom 2 Desktop Activity Levels..................................... 128

Figure 7.4: Utilization of Multiple Cores by SYSmark 2007 Benchmark................. 129

Figure 7.5: Periodic Power Phase Predictor.............................................................. 132

Figure 7.6: Example of Program Phase Mapping to Predictor ............................... 133

Figure 7.7 Core-Level Phase Length Probability Distributions ................................ 140

Figure 7.8 Prediction Accuracy of Core Power for Various Predictors..................... 144 


\section{Chapter 1 Introduction}

Computing systems have a wide range of design objectives. Metrics such as performance, power and cost must be carefully managed in order to meet these objectives. While some parameters are fixed at design time, others such as performance and power consumption may be dynamically adjusted at run-time. This allows a system to be optimal across a wider range of workloads and usage scenarios. This dynamic optimization, commonly known as dynamic power management, allows performance to be exchanged for power savings. The amount of savings is constrained by the system objectives. For example, systems with quality of service (QoS) requirements can allow power and performance to be reduced only as long as the service demands are met. Mobile systems powered by batteries must be optimized to deliver the highest performance/Watt in order to maximize usage time. Compute-cluster performance capacity must be modulated to match demand so that performance/cost is maximized. Adaptation within these scenarios requires accurate, run-time measurement of performance and power consumption. Run-time measurement of power and performance allow tradeoffs to be made dynamically in response to program and usage patterns.

\subsection{Attributing Power in Multi-Core Systems}

Multi-core and multi-threaded systems present significant challenges to power measurement. While performance is readily measurable at the core and program-level, 
power is more difficult. Invariably power is delivered to multiple cores or system components through a shared power plane. Power consumption by individual cores or programs cannot be observed. Consider Figure 1.1. The power consumption of a multicore, multi-programmed system simultaneously executing four distinct workloads is shown. These four workloads have power and performance characteristics that require distinct adaptations to meet system objectives. Core 1 has the computationally efficient ray-tracing workload, povray that scales performance nearly perfectly with core frequency. This is important for power management since controlling adaptations such as frequency scaling requires accounting for the potential benefit or cost of changing frequency. In contrast cores 0,2 and 3 are running applications that are sensitive to 58\%$80 \%$ of the change in core frequency. This difference in frequency sensitivity leads to varying optimization points for dynamic adaptation. Similarly, the power consumption of each workload is distinct. The highly efficient povray is able to consistently utilize more than $2 / 3$ of the execution pipelines. This high utilization and concentration of floating point instructions, leads to high power consumption. At the other extreme, the gcc compiler application is only able to utilize $1 / 3$ of the execution pipelines using integer instructions exclusively. In addition to differences in steady state power consumption, these workloads have different phase behavior. While povray and gcc have stable power consumption patterns, 3Dsmax and Sketchup (3D rendering) exhibit drastic variations in power consumption over time. Frequent changes in power consumption 
increases the overhead of adaptation since transition costs cannot be amortized in the short duration phases.

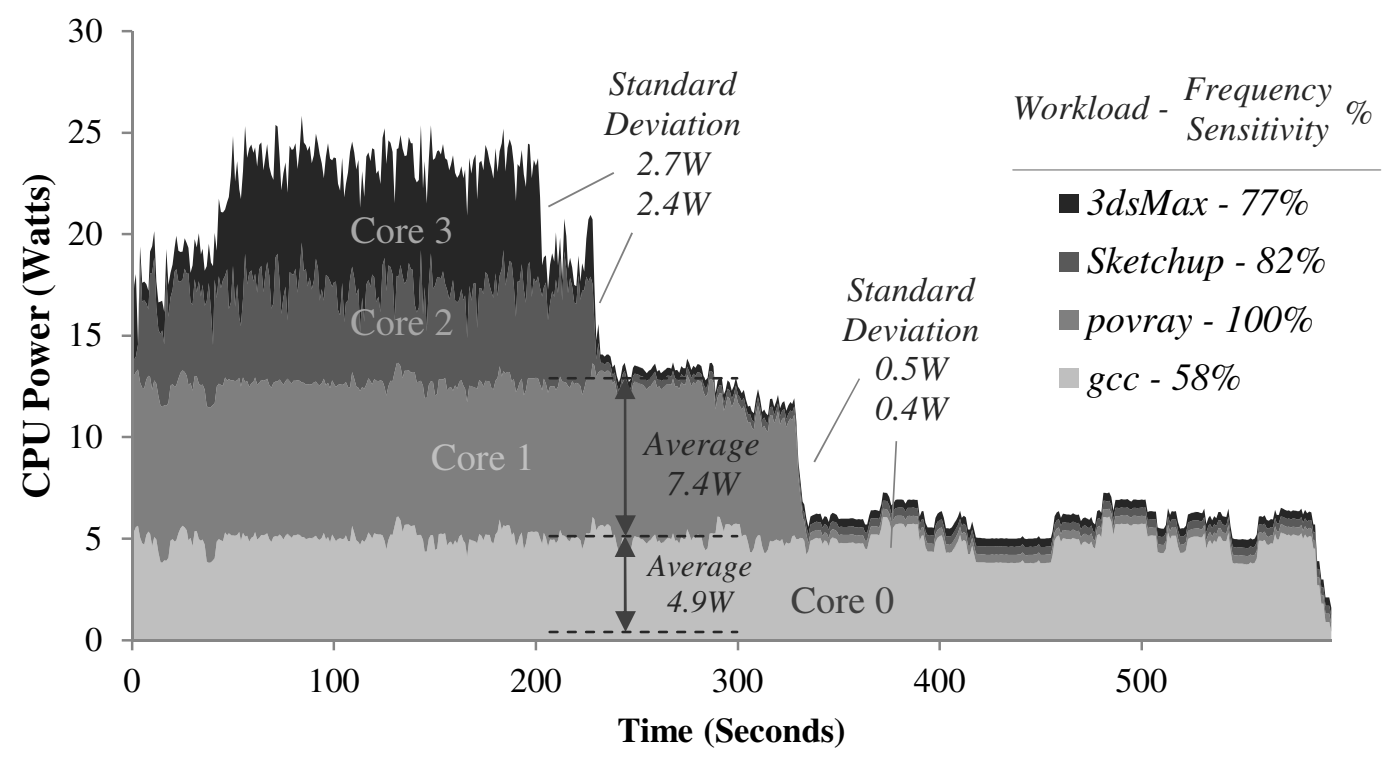

Figure 1.1 CPU Core-Level Power Accounting

The problem of multiple programs sharing power resources is not limited to processors. Attributing power consumption by a program within an entire system presents a similar challenge. Consider Figure 1.2, which illustrates power consumption for of a modern laptop computer system across a range of critical workloads. Similar to CPU cores, the power consumption in memory, chipsets, graphics and hard disks varies drastically across workloads. Effective power management requires that power be attributable to programs across the entire system so that power performance tradeoffs can be made. 


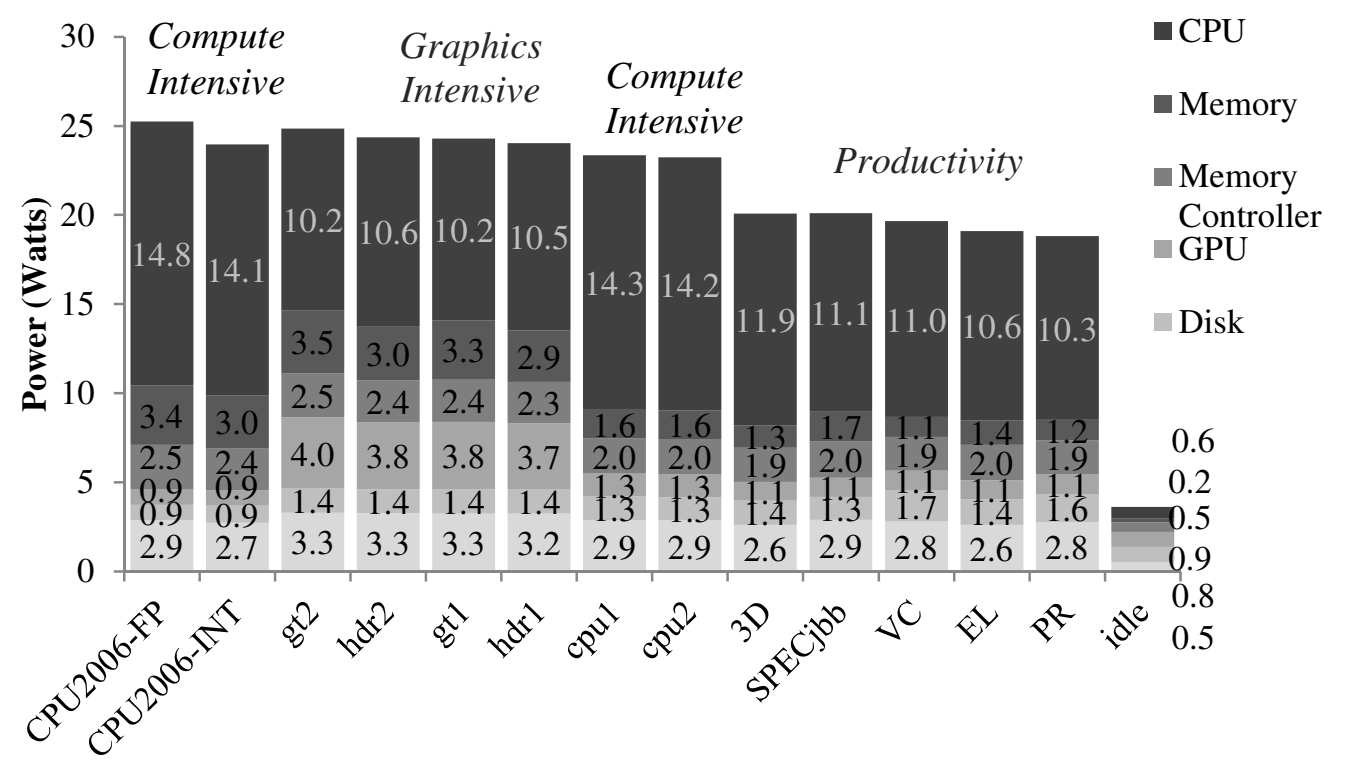

Figure 1.2 System-Level Power Accounting

\subsection{When to Adapt}

Due to the difficulty in observing program phases in shared power plane environments, existing power management schemes rely on reaction when performing adaptation. This pragmatic approach leads to sub-optimal performance and power consumption. Consider the case of the Windows Vista operating system using Dynamic Voltage and Frequency Scaling (DVFS). To reduce power consumption during low utilization phases, the operating system power manager reduces voltage and frequency of cores when CPU core activity level drops below a fixed threshold. The manager periodically samples core activity level and adjusts the DVFS operating point accordingly. This reactive approach results in frequent over and under provisioning of performance and power, especially for "bursty" workloads. Consider Table 1.1, which shows DVFS residency for a recent productivity workload. This data indicates that the selection of CPU frequency is 
suboptimal from a power and performance perspective. Though the CPU is capable of drastically reducing idle power by operating at less than maximum frequency, it frequently does not. On average $41 \%$ of idle time is spent at a higher than necessary frequency and voltage. Similarly, performance is reduced by operating at less than the maximum frequency for $70 \%$ of the time. This can greatly impact performance due to the large increases in runtime that can eliminate energy efficiency gains from reduced voltage and frequency.

To improve performance and efficiency, adaptation must be performed with an accurate estimate of future demand. This prevents costly adaptations from being applied when program phase are too short to amortize the performance and energy cost of adaptation. To this end a predictive power manager is proposed.

Table 1.1 Windows Vista Reactive DVFS

\begin{tabular}{|c|c|c|c|c|}
\hline & E-Learning & Productivity & Video Creation & $3 \mathrm{D}$ \\
\hline $2.4 \mathrm{GHz}-$ Active & $4.6 \%$ & $2.4 \%$ & $5.3 \%$ & $15.0 \%$ \\
\hline $2.4 \mathrm{GHz}-$ Idle & $17.4 \%$ & $9.6 \%$ & $12.4 \%$ & $17.0 \%$ \\
\hline $1.6 \mathrm{GHz}-$ Active & $1.4 \%$ & $0.8 \%$ & $3.2 \%$ & $5.1 \%$ \\
\hline $1.6 \mathrm{GHz}-$ Idle & $9.4 \%$ & $6.2 \%$ & $9.6 \%$ & $6.7 \%$ \\
\hline $1.2 \mathrm{GHz}-$ Active & $1.2 \%$ & $1.2 \%$ & $4.8 \%$ & $3.1 \%$ \\
\hline $1.2 \mathrm{GHz}-$ Idle & $9.8 \%$ & $9.7 \%$ & $13.8 \%$ & $9.4 \%$ \\
\hline $0.8 \mathrm{GHz}-$ Active & $4.5 \%$ & $4.7 \%$ & $4.8 \%$ & $6.9 \%$ \\
\hline $0.8 \mathrm{GHz}-$ Idle & $51.8 \%$ & $65.3 \%$ & $46.1 \%$ & $36.7 \%$ \\
\hline Active Frequency & $1.56 \mathrm{GHz}$ & $1.34 \mathrm{GHz}$ & $1.51 \mathrm{GHz}$ & $1.77 \mathrm{GHz}$ \\
\hline Idle Frequency & $1.24 \mathrm{GHz}$ & $1.07 \mathrm{GHz}$ & $1.20 \mathrm{GHz}$ & $1.32 \mathrm{GHz}$ \\
\hline
\end{tabular}




\subsection{Power Variation is Periodic}

The major challenge in predictive power management is detecting patterns of usage that are relevant for power adaptations. Fortunately, the most critical usage metric in modern computing systems is architecturally visible, namely CPU active/idle state usage. CPU active and idle states have been shown to be highly correlated to power and performance

demand in complete systems [BiJo06-1]. This allows power and performance demand in the complete system to be tracked and predicted, using only CPU metrics.

Since the predicted metrics are contained within the CPU, patterns are easily detectable. Consider Figure 1.3. It shows active and idle usage patterns during the playback of a Blu-Ray video. Due to the requirement for regular, periodic frame updates, the CPU active and idle patterns are also regular. Note the active decrypt/render phases that typically last about $10 \mathrm{~ms}$. This corresponds to the execution time to decrypt and render about six frames of video. In addition to these workload-dependent phases, there are also operating system induced phases. Interspersed with the long active phases are numerous, short, $1 \mathrm{~ms}$ phases. These phases are composed of slightly less than 100us active phases followed by $1 \mathrm{~ms}$ idle phases. The active phases are caused by the operating system scheduler waking the CPU to check for threads that are ready to execute. The regular patterns are ideal for prediction. 


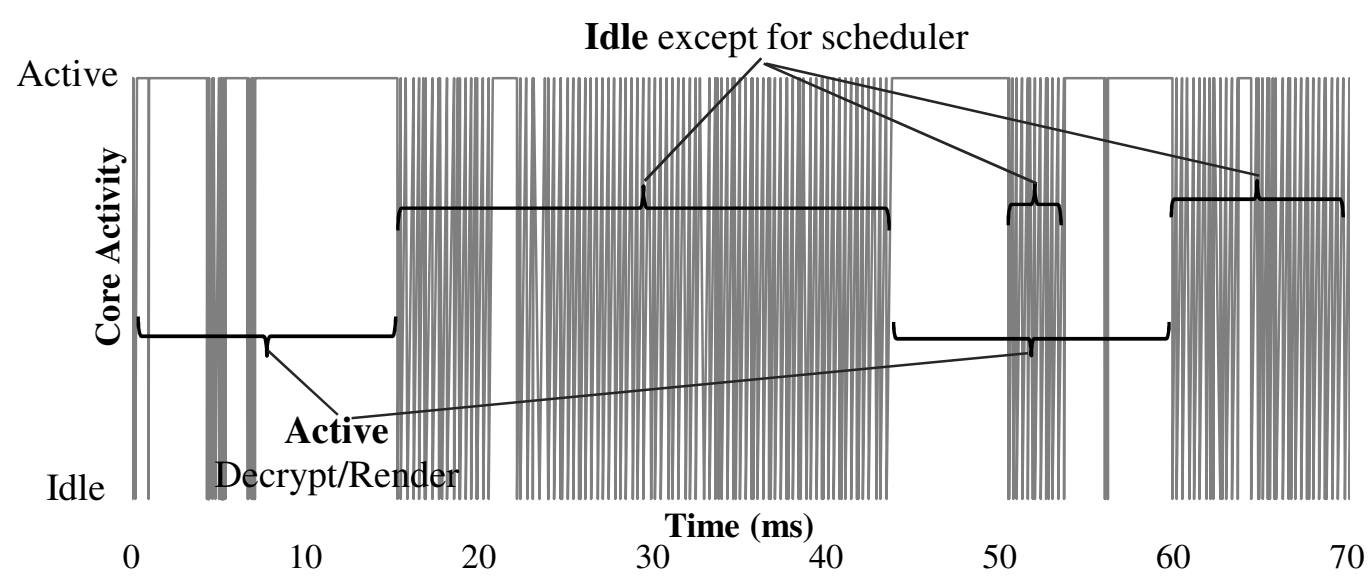

Figure 1.3 Core Activity Patterns - Blu-Ray Playback

Detecting patterns at the CPU-level is also advantageous since it allows component-level patterns to be discerned from aggregate patterns. Consider Figure 1.4. The top figure shows total CPU power consumption for a multi-core processor. The core-level power consumption is shown in the subsequent four figures. Though the individual cores have a regular, easily detectable pattern, the aggregate power obscures much of the periodic behavior. This concept extends to the complete system in which individual core or thread usage patterns induce similar patterns in shared resources such as memory or I/O devices. Tracking and predicting CPU usage patterns provides the opportunity to more effectively adapt power and performance to match demand of the complete system. 

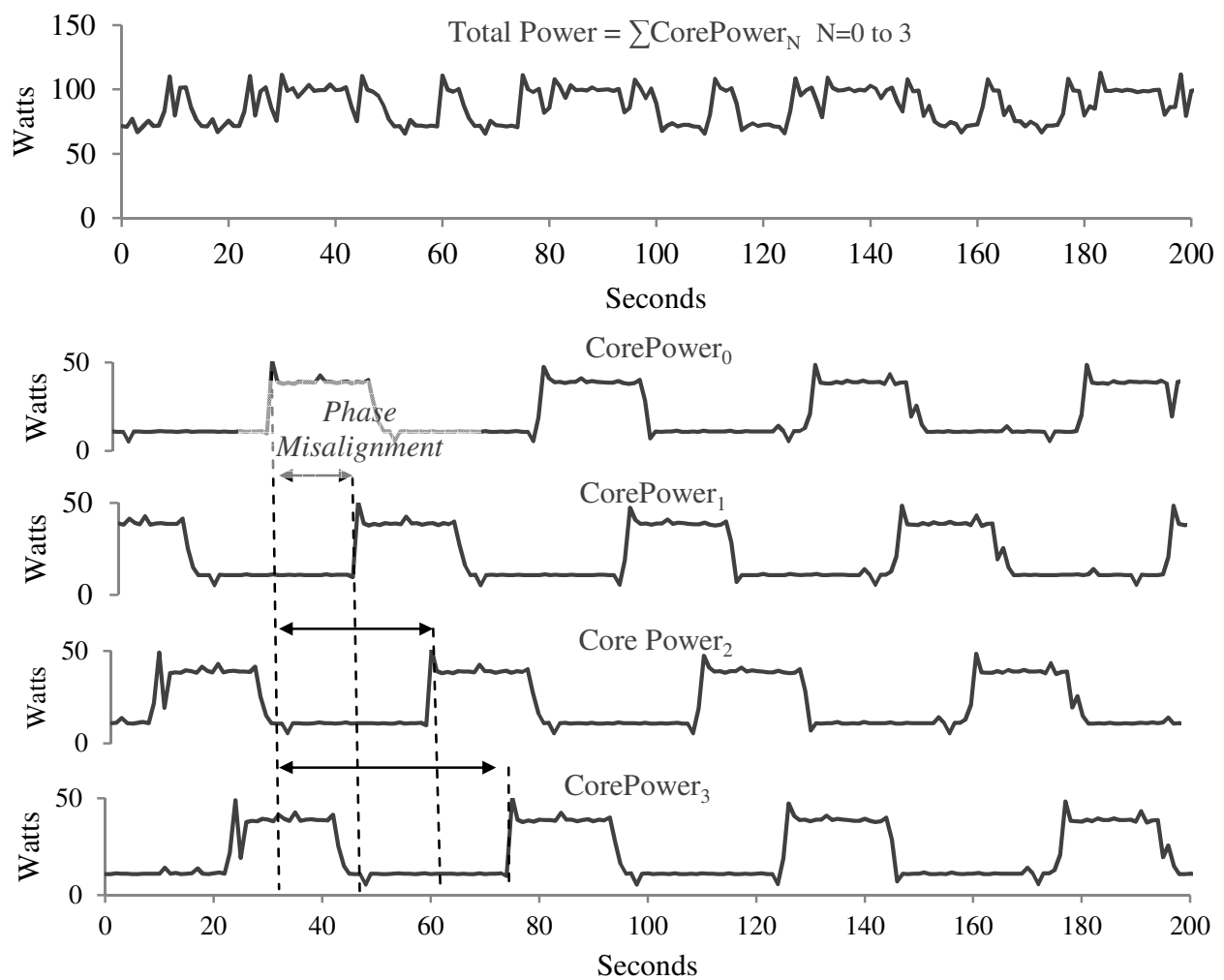

Figure 1.4 Thread and Aggregate Power Patterns

\subsection{Objectives}

The objective of this dissertation is to develop a predictive power manager using performance counter-based power models. The specific objectives are as follows.

1. Develop performance counter-based, run-time models for complete system power consumption.

2. Demonstrate the effectiveness of thread-level power accounting.

3. Characterize power consumption patterns of server, desktop and mobile systems. 
4. Design a predictive power management scheme to improve performance and power efficiency.

\subsection{Thesis Statement}

Complete system power consumption of a multi-core system can be accurately estimated by tracking core-level CPU performance events. These estimates may be used to predict changes in power and performance of the system. Compared to commercial, reactive power managers this predictive power manager yields higher performance and lower power.

\subsection{Contributions}

This dissertation makes several contributions in the areas of modeling methodology, power models, measurement-based workload characterization and novel power management strategies.

a) A methodology for constructing power models based on performance events. The methodology is shown to be effective across CPU architectures, system categories and workloads.

b) A simple, accurate model for CPU power based on performance counters. The model is constructed by applying linear-regression to power and performance measurements captured on an actual system. The model demonstrates the need to account for speculative execution when modeling power consumption. 
c) The concept of trickle-down power events is presented. By identifying CPU performance events that trickle-down to other subsystems, a complete-system power model based on CPU performance counters. Power for subsystems including memory, chipsets and disk are modeled using events directly measureable in the CPU.

d) A characterization of complete-system power consumption for server, desktop and mobile platforms is presented. The impacts of workloads, power management and temperature are quantified. Statistical characterization of power amplitude and duration is provided for numerous subsystems.

e) An analysis of the performance impacts on power management for multi-core processors. Performance loss due to power management of shared resources is considered. Certain workloads are found to be more sensitive to power management. Negative interactions between operating systems are shown to reduce performance and power efficiency.

f) A predictive power manager is proposed for controlling DVFS in a multi-core processor. By identifying and anticipating patterns of power consumption, the manager is able to improve performance and efficiency. It is compared to the commercial, reactive scheme used in Windows Vista. 


\subsection{Organization}

This dissertation is organized as follows:

Chapter 2 describes the methodology for measuring power and performance events within a range of system types and subsystems ranging from CPUs to hard drives. Techniques are provided for isolating and measuring dynamic and static power within actual computing systems.

Chapter 3 presents an analysis of processor performance events that correlate to power consumption. These findings direct the construction of a simple, speculationaware power model based on a small number of performance events. A formal methodology for developing performance counter power models is presented.

Chapter 4 provides a broad analysis of subsystem-level power consumption across a wide range of workloads including scientific computing, commercial transaction processing, desktop productivity, content creation and consumption. Power is considered in relative terms comparing across each subsystem. To inform power management decisions, power phase behavior is considered in terms of duration and amplitude.

Chapter 5 presents an extensive number of system power models based upon processor performance counters. The motivation behind the composition of each model is provided. Accuracy statistics and measure versus modeled time-domain comparisons are given. 
Chapter 6 explores the power and performance impact of dynamic power management. Detailed analysis of the relationship between power adaptations such as clock gating and DVFS and performance are provided. The sub-optimal nature of a commercial DVFS scheme is explored and explained.

Chapter 7 presents the Period Power Phase Predictor for control DVFS power management actions. The predictor is compared to a state-of-the-art commercial DVFS scheme in terms of performance and power consumption. Results are presented for a desktop productivity workload that contains a high-level of power phase transitions.

Chapter 8 summarizes previous contributions in the area performance counter power modeling and predictive power management. Chapter 9 describes conclusions topics of future research. 


\section{Chapter 2 Methodology}

The development of power models based on performance events requires the measurement of power and performance on systems running a wide range of workloads. This chapter describes the methodology for measuring power and performance events on actual systems (not simulation) running realistic workloads. The first section describes techniques and equipment for in-system measurement of power across a range of systems and components. The compositions of three systems are defined: server, desktop and laptop. The second section shows how system parameters such as temperature, voltage and frequency can be manipulated to expose and quantify underlying properties of systems. The third section describes how performance monitoring counters (PMC) can be tracked in a manner that has minimal impact on the observed system. The last section describes which workloads are preferred for power management analysis and why.

\subsection{Measuring System and Component Power}

To measure power consumption, a range of instrumentation methodologies are used. Each methodology is designed to match measurement requirements while conforming to the constraints of the measured system. The systems and measurement requirements are: 1) aggregate CPU power in a desktop system, 2) subsystem-level power in a server system, 3) subsystem-level power in a mobile system. 


\subsubsection{Aggregate CPU Power Measurement}

CPU power consumption is measured using a clamp-on current probe. The probe, an Agilent 1146A [Ag08], reports current passing through its sensor by detecting the magnitude and polarity of the electromagnetic field produced by the sampled current. This type of measurement simplifies instrumentation since the observed conductors do not have to be cut to insert current sensing resistors. The drawback of this approach is that only wire-type conductors can be sampled. It is not possible to sample conductors embedded in the printed circuit board. For the target system this restricts power measurement to the input conductors of the processor voltage regulator module (VRM). As a result, a portion of the reported power consumption is actually attributed to the inefficiency of the VRM. These modules have an efficiency of 85\%-90\%. The reader should consider the $10 \%-15 \%$ loss when comparing results to manufacturer reported power consumption. The voltage provided by the current probe is sampled at $10 \mathrm{KHz}$ by a National Instruments AT-MIO-16E-2 data acquisition card[Ni08]. The LabVIEW software tool [La10] can interpret the voltage trace or as in this case it is written to a binary file for offline processing. The details of the system are described below in Table 2.1 .

\section{Table 2.1 Desktop System Description}

\section{System Parameters}

Single Pentium 4 Xeon $2.0 \mathrm{GHz}$, 512KB L2 Cache, 2MB L3 Cache, 400 MHz FSB

4 GB PC133 SDRAM Main Memory

Two 16GB Adaptec Ultra160 10K SCSI Disks

\section{Redhat Linux}




\subsubsection{Subsystem-Level Power in a Server System}

To study component-level server power, the aggregate CPU power measurement framework is used and extended to provide additional functionality required for subsystem-level study. The most significant difference between the studies of CPU level versus subsystem level is the requirement for simultaneously sampling multiple power domains. To meet this requirement the IBM $\times 440$ server is used which provides separate, measureable power rails for five major subsystems. It is described in Table 2.2.

\section{Table 2.2 Server System Description}

\begin{tabular}{|c|} 
System Parameters \\
\hline Four Pentium 4 Xeon 2.0 GHz, 512KB L2 Cache, 2MB L3 Cache, $400 \mathrm{MHz}$ FSB \\
\hline 32MB DDR L4 Cache \\
\hline 8 GB PC133 SDRAM Main Memory \\
\hline Two 32GB Adaptec Ultra160 10K SCSI Disks \\
Fedora Core Linux, kernel 2.6.11
\end{tabular}

By choosing this server, instrumentation is greatly simplified due to the presence of current sensing resistors on the major subsystem power domains. Five power domains are considered: CPU, chipset, memory, I/O, and disk. The components of each subsystem are listed in Table 2.3.

Table 2.3 Subsystem Components

\begin{tabular}{|c|c|}
\hline Subsystem & Components \\
\hline CPU & Four Pentium 4 Xeons \\
\hline Chipset & Memory Controllers and Processor Interface Chips \\
\hline Memory & System Memory and L4 Cache \\
\hline I/O & I/O Bus Chips, SCSI, NIC \\
\hline Disk & Two 10K rpm 32G Disks \\
\hline
\end{tabular}


Power consumption for each subsystem (CPU, memory, etc.) can be calculated by measuring the voltage drop across each current sensing resistor. In order to limit the loss of power in the sense resistors and to prevent excessive drops in regulated supply voltage, the system designer used a particularly small resistance. Even at maximum power consumption, the corresponding voltage drop is in the tens of millivolts. In order to improve noise immunity and sampling resolution we design a custom circuit board [Bi06] to amplify the observed signals to levels more appropriate for the measurement environment. The printed circuit board is shown in Figure 2.1. This board provides amplification for eight current measurement channels. The board also provides BNCtype connecters to allow direct connection to the data acquisition component. The entire measurement framework is shown in Figure 2.2.

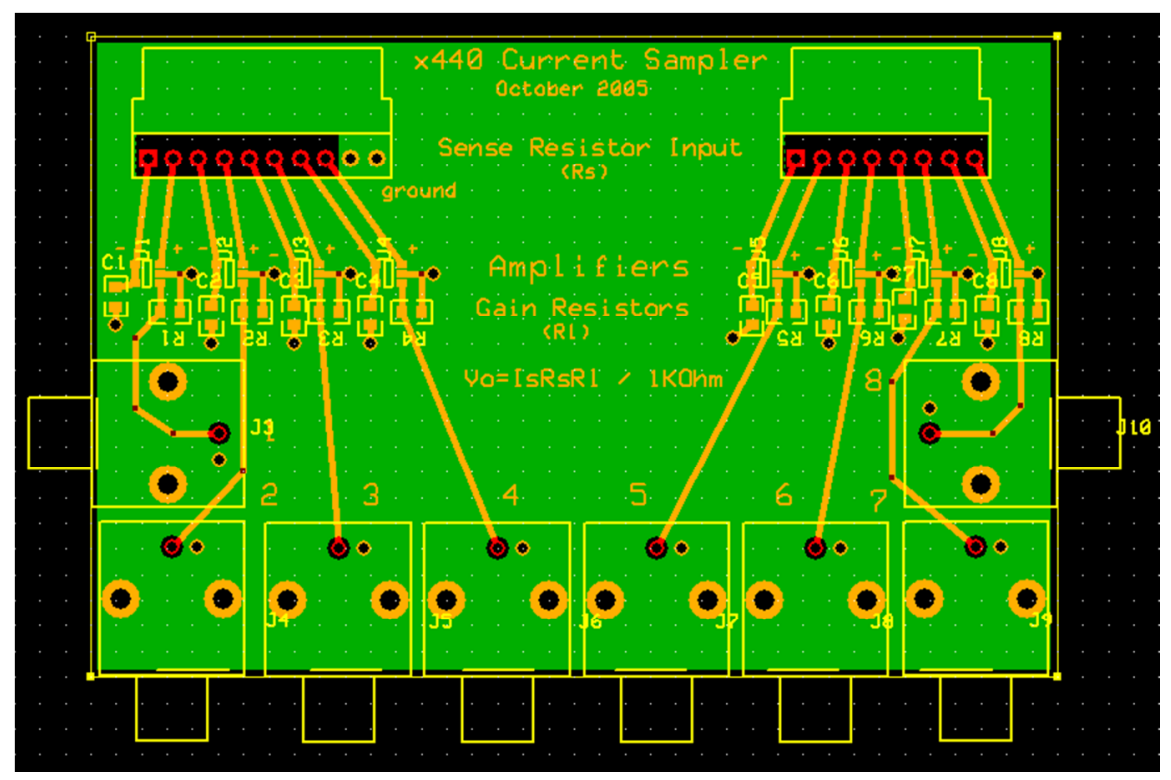

Figure 2.1 Current Sense Amplification PCB 
The main components are subsystem power sensing, amplification (current probe), data acquisition, and logging. Subsystem power sensing is provided by resistors onboard the $\mathrm{x} 440$ server. The voltage drop across the resistors is amplified by the custom circuit board. The amplified signals are captured by the data acquisition card. Finally, the host system, running LabVIEW, logs the captured data to a file for offline processing.

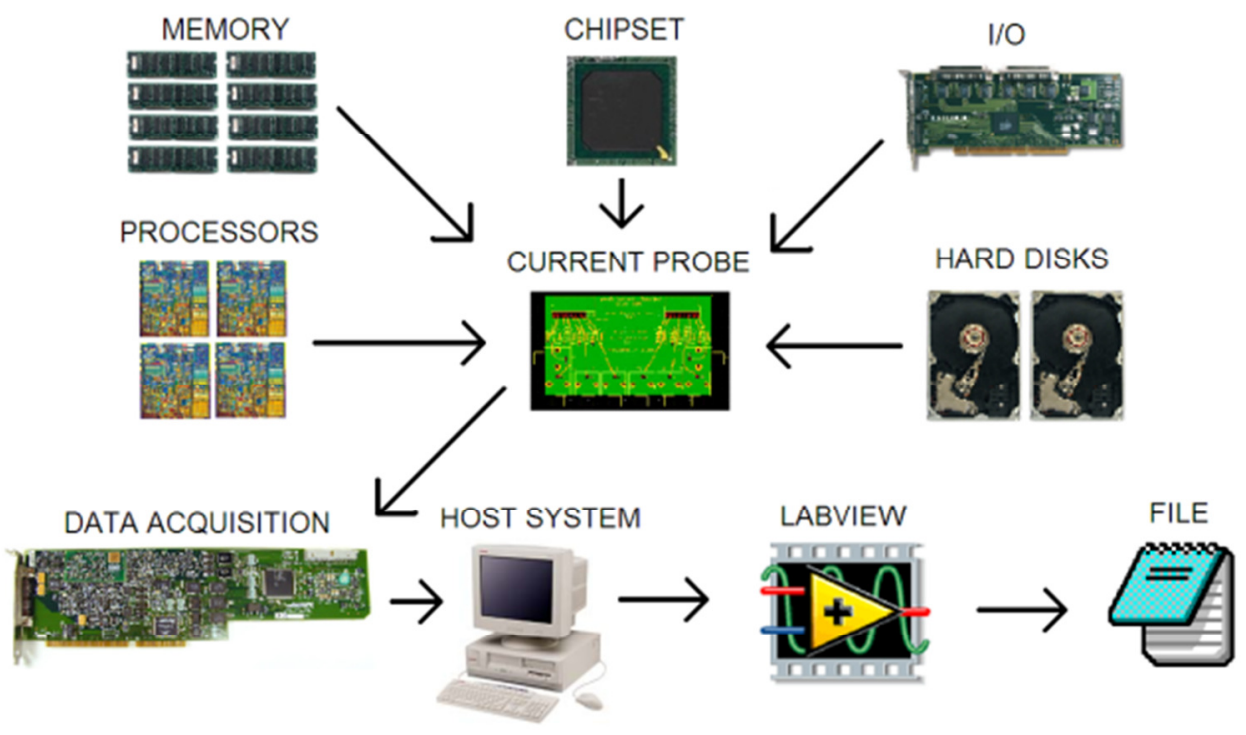

Figure 2.2 Power Measurement Environment

\subsubsection{Subsystem-Level Power in a Mobile System}

Power measurement at the subsystem-level in a mobile system presents unique opportunities and challenges not typically encountered in desktop or server systems. Due to the requirement for low power consumption and long battery-life, mobile systems implement an extensive array of power saving features. These features require isolation of power delivery so subsystems can be managed independently. This isolation allows 
power to be measured at a finer grain than desktop/server systems that have a larger degree of sharing across subsystems. It also leads to a wider range of power levels across subsystems. High-power CPU subsystems may typically consume tens of Watts while low-power chipsets may only consume a Watt or less. The measurement of different ranges of power requires different approaches in order to maximize accuracy and minimize perturbation. To this end a system specifically designed for power analysis is used. The system is used by a major CPU manufacturer [Bk09] in the validation of processors and chipsets. Depending on the expected power levels for a given subsystem an inline current sensing resistor is implemented. High current subsystems use low value resistors in the range of just a few milliohms. Low current subsystems use resistors in the range of a few hundred milliohms. This approach allows the observed voltage drop due to current flow to always be within the maximum accuracy range of the data acquisition device. It also reduces the impact measurement has on the system. If the voltage drop due to the sensor is too large, the effective voltage delivered to the subsystem could be out of the subsystem's operating range. The system characteristics and measureable subsystems are listed below in Table 2.4. 
Table 2.4 Laptop System Description

\begin{tabular}{|c|c|}
\hline Processor(s) & Dual-core 45nM 2.0GHz \\
\hline Memory & 4GB DDR3-1066 \\
\hline & CPU Clock Gating and DVFS \\
Power & DRAM Power Down and Self Refresh \\
Management & Chipset Link Disconnect \\
& Harddrive Spin Down and ATA modes \\
Graphics Processor Clock Gating
\end{tabular}

\subsection{Controlling Temperature, Voltage and Frequency}

The development of power and performance models that are sensitive to temperature, voltage and frequency requires those parameters to be independently controlled. To this en`d, multiple techniques are employed. The most difficult parameter to control is temperature. Temperature has a major impact on power consumption due to its exponential relationship with leakage power. Depending on the intensity, instruction mix and data use pattern of workloads, temperature and therefore power varies drastically. To eliminate this effect a closed loop temperature controller is used to regulate processor package temperature. The controller regulates temperature within 0.1 degree Celsius from $20 \mathrm{C}$ to $100 \mathrm{C}$. It circulates chilled, 20C water to remove heat from the processor package. Fine-grain control of temperature is provided by a Peltier-effect thermoelectric cooler. This device can rapidly add or remove heat from the processor package depending on demand. Workloads that naturally heat the processor above the setpoint, 
cause the controller to remove the excessive heat. Workloads operating below the setpoint cause it to add heat. The controller is able to dynamically adjust the heating or cooling load with changes in the workload. This fine-grain control of temperature provides two important abilities: isolation of leakage from switching power and development of temperature sensitive leakage model.

Voltage and frequency control are provided through architectural interfaces provided in the processor. Recent processors [Bk09] provide architectural control of processor core frequency and voltage through model specific registers. This interface allows systemlevel code to create arbitrary combinations of voltage and frequency operating points for DVFS and clock gating functions. Fixing voltage and scaling frequency allows calculation of leakage power. See Figure 2.3. Fixing frequency and scaling voltage and temperature allows the derivation of voltage and temperature-dependent leakage models.

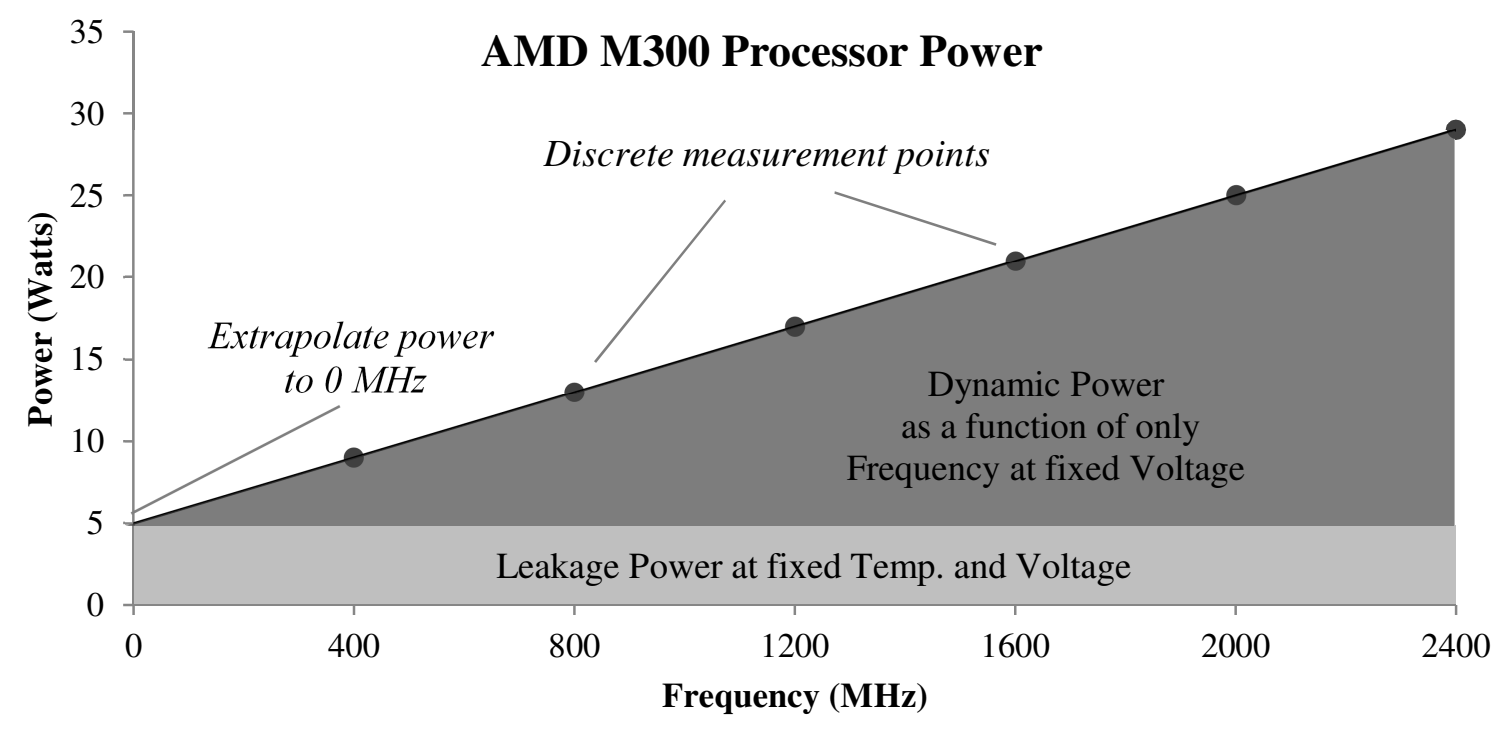

Figure 2.3 Leakage Power Determination 


\subsection{Performance Counter Sampling}

To sample performance monitoring counters a small kernel that provides periodic sampling of processor performance counters is developed. This kernel uses a device driver to provide ring- 0 access to user-mode applications. This approach is preferred over existing user-mode performance counter libraries as it affords more precise control of sampling and lower overhead. In all experiments, the worst-case sampling overhead (\% CPU time sampling) for performance counter access averages less than $1 \%$ for sampling intervals as low as $16 \mathrm{~ms}$. In addition to the performance impact of counter sampling, there is a power impact which must be minimized. A common problem with periodically scheduled code, such as performance counter sampling, is excessive scheduler activity. This activity causes CPUs to frequently exit the idle state to service interrupts, thus increasing power consumption. The sampling kernel avoids this issue by explicitly requesting a scheduling interval that exactly matches the required sampling interval. As a result the scheduler only runs enough to schedule the performance counter sampling events and background operating system activity.

\subsection{Workloads}

Workload selection is a critical part of dynamic power management analysis. The focus on power accounting and prediction requires workloads with widely varying power and performance levels. Unlike microarchitectural analysis that considers phases within an 
instruction stream lasting only a few microseconds, dynamic power management must also consider long duration phases ranging from hundreds to millions of microseconds. These phases, caused by events such as thread migrations, context switches or device interrupts provide greater opportunity (and challenge) for power management due to the longer time for amortizing adaptation costs. To this end, this dissertation analyzes power consumption, modeling and prediction across over sixty distinct subtests. The workloads and their characteristics are listed in Table 2.5.

Table 2.5 Workload Description

\begin{tabular}{|c|c|c|c|c|}
\hline $\begin{array}{c}\text { Name } \\
\text { [Subtest Count] }\end{array}$ & $\begin{array}{l}\text { Workload } \\
\text { Type }\end{array}$ & $\begin{array}{l}\text { Subsystem } \\
\text { Target }\end{array}$ & Phase Behavior & $\begin{array}{l}\text { Systems } \\
\text { Analyzed }\end{array}$ \\
\hline $\begin{array}{c}\text { SPEC CPU } 2000 \\
{[26]}\end{array}$ & Scientific & $\begin{array}{c}\text { CPU } \\
\text { DRAM }\end{array}$ & Instruction & $\begin{array}{l}\text { Server } \\
\text { Desktop } \\
\text { Laptop }\end{array}$ \\
\hline $\begin{array}{c}\text { SPEC CPU } 2006 \\
{[29]}\end{array}$ & Scientific & $\begin{array}{c}\text { CPU } \\
\text { DRAM }\end{array}$ & Instruction & $\begin{array}{l}\text { Server } \\
\text { Desktop } \\
\text { Laptop }\end{array}$ \\
\hline $\begin{array}{c}\text { SPECjbb } 2005 \\
{[1]}\end{array}$ & $\begin{array}{l}\text { Transaction } \\
\text { Processing }\end{array}$ & $\begin{array}{l}\text { CPU } \\
\text { DRAM }\end{array}$ & Instruction & Server \\
\hline $\begin{array}{c}\text { DBT-2 } \\
{[1]}\end{array}$ & Database & $\begin{array}{l}\text { I/O } \\
\text { Disk }\end{array}$ & $\begin{array}{c}\text { Instruction } \\
\text { Active-Idle } \\
\text { Power Management }\end{array}$ & Server \\
\hline $\begin{array}{c}\text { SYSmark } 2007 \\
{[4]}\end{array}$ & Productivity & $\begin{array}{c}\text { CPU } \\
\text { DRAM } \\
\text { I/O } \\
\text { Disk }\end{array}$ & $\begin{array}{c}\text { Instruction } \\
\text { Active-Idle } \\
\text { Threadedness } \\
\text { Power Management }\end{array}$ & $\begin{array}{l}\text { Desktop } \\
\text { Laptop }\end{array}$ \\
\hline $\begin{array}{c}\text { 3DMark } 2006 \\
{[6]}\end{array}$ & 3D Gaming & $\begin{array}{l}\text { Graphics } \\
\text { CPU } \\
\text { DRAM }\end{array}$ & $\begin{array}{c}\text { Instruction } \\
\text { Active-Idle } \\
\text { Power Management }\end{array}$ & Laptop \\
\hline $\begin{array}{l}\text { Idle } \\
{[1]}\end{array}$ & Idle & CPU & Active-Idle & $\begin{array}{l}\text { Server, } \\
\text { Desktop } \\
\text { Laptop }\end{array}$ \\
\hline
\end{tabular}

To develop power models for active execution (non-idle) the SPEC CPU 2000, 2006 and SPECjbb 2005 workloads are used [Sp00] [Sp06] [Sj06]. These workloads contain 
instruction streams that exercise a wide range of intensities in the CPU and memory subsystems. They include integer and floating centric workloads. Within these two types the focus varies from workloads completely bound by CPU execution speed to those bound be memory access latency and throughput. These benchmarks provide sufficient information to develop active power models for CPU and memory. The limitation is that they operate in an unrealistic fully-active mode utilization only the CPU and memory subsystems. Unlike real usage scenarios, these workloads do not frequently transition between the active and idle states or exercise disk, graphics or I/O subsystems.

To address this limitation the DBT-2, SYSmark 2007 and 3DMark 2006 benchmarks are included. These workloads emulate real usage scenarios by including user-input and system interactions. DBT-2 [Os06] is intended to approximate the TPC-C transaction processing benchmark. This workload does not require network clients, but does use actual hard disk access through the PostgreSQL [PS06] database. SYSmark 2007 [Sm07] is implemented using simulated user input through the application GUI (graphical user interface). The numerous delays required for GUI interaction causes many idle phases across the subsystems. This causes a large degree of active-idle and idle-active transitions, thread migrations and power management events. 3DMark06 [3d06] contains six subtests covering CPU and graphics-intensive workloads. Additionally, systems are characterized in the idle state. This sets a baseline for power consumption and represents common usage patterns. 


\section{Chapter 3 Modeling CPU Power using Performance Monitoring Counters}

Effective power management requires fine-grain accounting of power within complex, computing systems. Since these systems contain multiple components sharing power resources, it is difficult to attribute power to individual components. It has been shown that performance-relevant events are strong predictors of power consumption. Due to the widespread availability of on-chip performance monitoring facilities, it is possible to develop accurate, run-time power models based upon performance events. This chapter demonstrates the effectiveness of these at CPU power accounting. Models are shown ranging from simple three-term linear to polynomial models that account for power management and workload effects such voltage, frequency and temperature. The chapter concludes with a formal definition of the model building methodology.

\subsection{Correlation of Performance Counters to Power}

While past research [LiJo03] [Be00] and intuition suggest that instructions/cycle (IPC) alone can account for CPU power, this study considers a larger array of metrics for building models. Correlation coefficients were calculated for all twenty-one observed PMCs. Initially, we attempted to find correlation across multiple sample points in a single workload trace. However, it was found that minor discrepancies in alignment of 
the power trace to the PMC trace could cause large variations in correlation. Since there is such a large set of workloads each workload is used as a single data point in the correlation calculation. For each metric the average rate across each workload is determined. For most, the metrics are converted to event/cycle form, but a few are in other forms such as hit rates. Additional derived metrics are included such as completed $\mu$ ops/cycle (retired + cancelled $\mu$ ops). A subset of the correlation results can be seen in Table 3.1.

Table 3.1. Intel Pentium 4, High and Low Correlation Performance Metrics

\begin{tabular}{|l|c|}
\hline Metric & Correlation \\
\hline Speculatively Issued $\mu$ ops/Cycle & 0.89 \\
\hline Fetched $\mu$ ops/Cycle & 0.84 \\
\hline Retired Instructions/Cycle & 0.84 \\
\hline Completed $\mu$ ops/Cycle & 0.83 \\
\hline Loads/Cycle & 0.80 \\
\hline Retired $\mu$ ops/Cycle & 0.79 \\
\hline Branches/Cycle & 0.78 \\
\hline Stores/Cycle & 0.64 \\
\hline Mispredicted Branches/Cycle & 0.41 \\
\hline Level 2 Cache Misses/Cycle & -0.33 \\
\hline Cancelled $\mu$ ops/Cycle & 0.33 \\
\hline Level 2 Cache Hits/Cycle & 0.31 \\
\hline Bus Accesses/Cycle & -0.31 \\
\hline Trace Cache Issued $\mu$ ops/Cycle & 0.32 \\
\hline Bus Utilization & -0.31 \\
\hline Floating Point ops/ $\mu$ op & -0.22 \\
\hline Prefetch Rate & 0.17 \\
\hline Trace Cache Build $\mu$ ops/Cycle & -0.15 \\
\hline Instruction TLB Hits/Cycle & -0.09 \\
\hline Trace Cache Misses/Cycle & -0.09 \\
\hline Instruction TLB Misses/Cycle & -0.04 \\
\hline
\end{tabular}


As expected IPC-related metrics show strong correlation. One of the more unexpected findings is the weak negative correlation of floating point instruction density (ratio of all dynamic instructions). This is in contrast to past findings [Be00] that show a strong correlation between floating point operations per second and power. Later in section 3.3 an explanation is provided. Another unexpected result is the lack of correlation to data prefetch rate.

This research shows that rather than considering only IPC, a more accurate model can be constructed using a metric that encompasses power consumed due to speculation. Figure 3.1 shows the average number of $\mu$ ops for the SPEC 2000 benchmarks that are fetched, completed and retired in each cycle. Table 3.2 shows the portions of fetched $\mu$ ops that complete or retire, for each of the twenty-four benchmarks.

Table 3.2 Percent of Fetched $\mu$ ops Completed/Retired - SPEC CPU 2000

\begin{tabular}{|c|c|c|c|c|c|}
\hline Name & \%Complete & \%Retire & Name & \%Complete & \%Retire \\
\hline gzip & 92.7 & 69.8 & wupwise & 97.0 & 91.0 \\
\hline vpr & 85.3 & 60.0 & swim & 99.9 & 99.7 \\
\hline gcc & 94.2 & 77.7 & mgrid & 99.1 & 98.6 \\
\hline mcf & 63.0 & 31.5 & applu & 98.7 & 96.6 \\
\hline crafty & 94.6 & 78.4 & equake & 96.8 & 93.5 \\
\hline bzip2 & 92.0 & 72.1 & sixtrack & 99.2 & 97.8 \\
\hline vortex & 98.0 & 95.0 & mesa & 92.1 & 75.2 \\
\hline gap & 92.8 & 73.5 & art & 84.9 & 77.5 \\
\hline eon & 91.7 & 81.5 & facerec & 95.5 & 90.5 \\
\hline parser & 90.1 & 69.0 & ammp & 94.8 & 88.5 \\
\hline twolf & 85.2 & 55.2 & fma3d & 97.0 & 94.3 \\
\hline & & & lucas & 99.9 & 95.9 \\
\hline & & & apsi & 97.1 & 93.6 \\
\hline Integer Avg. & 88.7 & 69.4 & Float Avg. & 96.3 & 91.7 \\
\hline
\end{tabular}


The first bar in Figure 3.1 "Fetch" shows the number of $\mu$ ops that are fetched from the Trace Cache in each cycle. The second bar "Complete" shows the sum of $\mu$ ops that are either retired or cancelled each cycle. Cancelled $\mu$ ops are due to branch misprediction. The third bar, "Retire", shows only $\mu$ ops that update the architectural state. This figure shows that the processor fetches $21.9 \%$ more $\mu$ ops than are used in performing useful work. Therefore, a more accurate power model should use the number of $\mu$ ops fetched per cycle instead of the number retired. Table 3.3 provides a comparison of linear regression power models based on these three metrics.

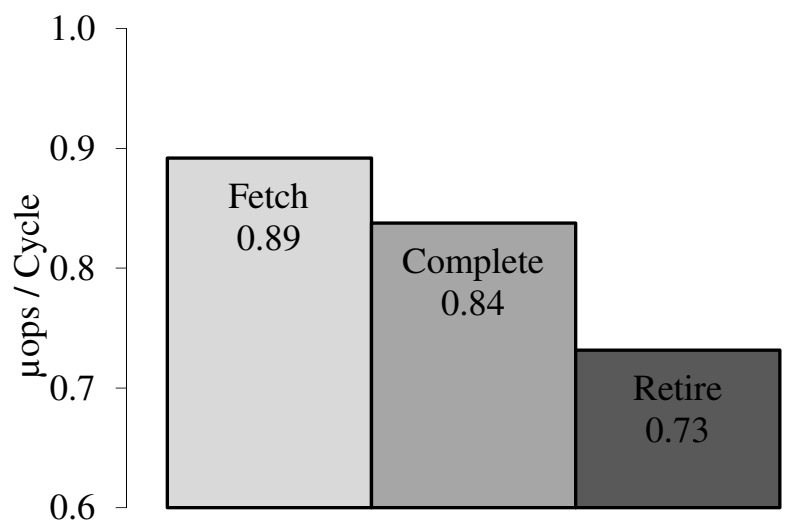

Figure 3.1. Average $\mu$ Ops/cycle - SPEC CPU 2000

\subsection{IPC Related Power Models}

Twenty-one processor performance metrics are examined for their correlation to power consumption. The most correlated metrics are all similar to (retired) instructions per cycle. Using this finding as a guide numerous linear models are constructed using regression techniques. Power is calculated as the sum of a positive constant $\alpha_{0}$ and the 
product of another positive constant $\alpha_{1}$ and a performance metric metric ${ }_{1}$. An example is shown in Equation 3.1.

$$
\text { Power }=\sum_{i=0}^{N} \alpha_{i} \times \text { metric }_{i}+\alpha_{i+1} \times \text { metric }_{i+1} \ldots \alpha_{N} \times \text { metric }_{N}
$$

Results for seven of the best models are listed below in Tables 3.3, 3.4 and 3.6. Tables 3.3 and 3.6 support the hypothesis that fetched $\mu$ ops are the most representative of IPC type metrics. The worst of these metrics is the familiar IPC. This is caused by the lack of a one-to-one mapping of instructions to $\mu$ ops. Many x86 instructions map to a sequence of $\mu$ ops. For example, a single ADD instruction that uses memory as its source and destination is actually composed of three $\mu$ ops. The first $\mu$ op loads a value from memory, the second adds a register or immediate to the value from memory and the third stores the result back to memory. Alternatively, an ADD instruction that does not use memory as an operand has a one-to-one mapping of instruction to $\mu$ op. Assuming all $\mu$ ops consume the same amount of power, the instruction that uses memory would consume three times as much power.

\section{Table 3.3 $\mu$ op Linear Regression Model Comparison}

\begin{tabular}{|c|c|c|c|c|c|c|}
\hline & \multicolumn{2}{|c|}{ Retired $\mu$ ops/cyc } & \multicolumn{2}{c|}{ Completed $\mu$ ops/cyc } & \multicolumn{2}{c|}{ Fetched $\mu$ ops/cyc } \\
\hline \multirow{2}{*}{ Coefficients } & $\alpha_{0}$ & $\alpha_{1}$ & $\alpha_{0}$ & $\alpha_{1}$ & $\alpha_{0}$ & $\alpha_{1}$ \\
\hline & 36.3 & 4.37 & 35.8 & 4.44 & 35.7 & 4.31 \\
\hline Avg Error & \multicolumn{2}{|c|}{$3.26 \%$} & \multicolumn{2}{|c|}{$2.8 \%$} & \multicolumn{2}{|c|}{$2.6 \%$} \\
\hline $\begin{array}{c}\text { Coefficient of } \\
\text { Determination }\end{array}$ & \multicolumn{2}{|c|}{0.696} & \multicolumn{2}{|c|}{0.735} & \multicolumn{2}{|c|}{0.737} \\
\hline
\end{tabular}


Table 3.4 Instruction Linear Regression Model Comparison

\begin{tabular}{|c|c|c|c|c|}
\hline & \multicolumn{2}{|c|}{$\begin{array}{c}\text { Retired } \\
\text { instructions/cyc }\end{array}$} & \multicolumn{2}{|c|}{$\begin{array}{c}\text { Completed } \\
\text { instructions /cyc }\end{array}$} \\
\hline Coefficients & $\alpha_{0}$ & $\alpha_{1}$ & $\alpha_{0}$ & $\alpha_{1}$ \\
\hline & 36.8 & 5.28 & 36.3 & 5.52 \\
\hline Avg Error & $5.45 \%$ & $4.92 \%$ \\
\hline $\begin{array}{c}\text { Coefficient of } \\
\text { Determination }\end{array}$ & 0.679 & 0.745 \\
\hline
\end{tabular}

Of the $\mu$ op-based models fetched $\mu$ ops is the most representative metric for power consumption. This suggests that $\mu$ ops that do not update the architected state of the machine still consume a significant amount of power. For the case of cancelled $\mu$ ops, this is not surprising since these $\mu$ ops did complete execution but were not retired. So, they would have traversed nearly the entire processor pipeline consuming a similar power level as retired $\mu$ ops. More surprising is the effect of fetched $\mu$ ops on the power model. Fetched $\mu$ ops includes retired and cancelled operations. It also includes the remaining $\mu$ ops that were cancelled before completing execution. Since fetched $\mu$ ops provides the most accurate model, cancelled $\mu$ ops must be consuming a significant amount of power.

These models generate minimum and maximum power values $(36 \mathrm{~W}-47 \mathrm{~W})$ similar to what was found on a Pentium 3 (31W-48W) [Be00] with similar $\mu$ op/cycle ranges $(0-$ 2.6). The stated average error values are found using the validation set described in Table 3.1. 


\subsection{Micro ROM Related Power Models}

The power models in Tables 3.3 and 3.4 perform best when applied to workloads mostly composed of integer-type instructions (SPEC-INT). Larger errors occur for workloads with high rates of floating point instructions (SPEC-FP). Isci et al [IsMa03] demonstrate that FP workloads such as equake use complex microcode ROM delivered $\mu$ ops. While the complex instructions execute, microcode ROM power consumption is high, but total power consumption is reduced slightly. In order to determine if this is the case for these traces, several synthetic workloads are created, composed almost entirely of complex instructions. Each of the programs is composed of a single large loop that is repeated for approximately ten seconds. The loop body is composed of numerous instances (30+) of one particular instruction. Since more than $90 \%$ of executed instructions are identical, average power due an individual instruction can be estimated.

\section{Table 3.5 Instruction Power Consumption}

\begin{tabular}{|c|c|c|c|}
\hline Instruction & $\begin{array}{c}\text { Power } \\
\text { (Watts) }\end{array}$ & $\begin{array}{c}\text { First Instruction Latency } \\
\text { (cycles) }\end{array}$ & $\begin{array}{c}\text { Subsequent Instruction Latency } \\
\text { (cycles) }\end{array}$ \\
\hline fcos & 30 & $180-280$ & 130 \\
\hline fsin & 31 & $160-200$ & 130 \\
\hline fptan & 25 & $240-300$ & 170 \\
\hline imul & 28 & $15-18$ & 5 \\
\hline idiv & 32 & $66-80$ & 30 \\
\hline
\end{tabular}

Table 3.5 [In04] shows that high latency instructions such as floating point type, consume less power than the $36 \mathrm{~W}$ minimum predicted by the IPC models. One possible cause is greater opportunity for clock gating. Since these instructions are guaranteed to take a 
long time to complete, more aggressive power saving techniques may be performed. Further investigation will be required to validate this hypothesis. Since Table 3.5 supports the conclusion that high latency instructions consume less power, the models can be improved by accounting for this behavior. One possible accounting method is to note that most high latency instructions are composed of relatively long $\mu$ op sequences sourced by the microcode ROM. Microcode ROM events can be observed using the trace cache metric, microrom $\mu$ ops. This metric counts the number of $\mu$ ops delivered from the microcode ROM. The resultant models are given in Table 3.6. As expected from the observations of power consumption of microcode ROM delivered instructions, the model's microcode ROM component is negative. This small correction allows the power model to extend below $36 \mathrm{~W}$ for workloads with high instances of complex microcode ROM instructions.

Table 3.6 $\mu$ op Linear Regression Model Comparison

\begin{tabular}{|c|c|c|c|c|c|c|c|}
\hline & \multicolumn{3}{|c|}{ Deliver, $\mu$ ROM } & \multicolumn{3}{c|}{ Deliver, $\mu$ ROM, Build } \\
\hline \multirow{2}{*}{ Coefficients } & $\alpha_{0}$ & $\alpha_{1}$ & $\alpha_{2}$ & $\alpha_{0}$ & $\alpha_{1}$ & $\alpha_{2}$ & $\alpha_{3}$ \\
\cline { 2 - 7 } & 36.7 & 4.24 & -11.8 & 36.7 & 4.24 & -14.6 & 5.74 \\
\hline Avg Error & \multicolumn{3}{|c|}{$2.50 \%$} & & $2.55 \%$ & \\
\hline $\begin{array}{c}\text { Coefficient of } \\
\text { Determination }\end{array}$ & \multicolumn{3}{|c|}{0.844} & & 0.850 \\
\hline
\end{tabular}

\subsection{Power Management Effects}

While instruction and $\mu$ op-based power models accurately account for power during fully active phases, they perform poorly in the presence of dynamic power management and temperature variation. This section characterizes the effect that power adaptations such 
as clock gating and dynamic voltage and frequency scaling have on power consumption. The strong relationship between temperature and leakage power is described. These findings are then used to develop a fully power management and temperature-aware processor power model.

\subsubsection{Active and Idle Power Management}

An effective power management strategy must take advantage of program and architecture characteristics. Designers can save energy while maintaining performance by optimizing for the common execution characteristics. The two major power management components are active and idle power management. Each of these components use adaptations that are best suited to their specific program and architecture characteristics. Active power management seeks to select an optimal operating point based on the performance demand of the program. This entails reducing performance capacity during performance-insensitive phases of programs. A common example would be reducing the clock speed or issue width of a processor during memory-bound program phases. Idle power management reduces power consumption during idle program phases. However, the application of idle adaptations is sensitive to program phases in a slightly different manner. Rather than identifying the optimal performance capacity given current demand, a tradeoff is made between power savings and responsiveness. In this case the optimization is based on the length and frequency of a program phase (idle phases) rather than the characteristics of the phase (memory-boundedness, IPC, cache miss rate). In the remainder of this section active power adaptations are referenced as p-states and idle 
power adaptations as $c$-states. These terms represent adaption operating points as defined in the ACPI specification. ACPI [Ac07] “...is an open industry specification codeveloped by Hewlett-Packard, Intel, Microsoft, Phoenix, and Toshiba. ACPI establishes industry-standard interfaces enabling OS-directed configuration, power management, and thermal management of mobile, desktop, and server platforms."

\subsubsection{Active Power Management: P-states}

A p-state (performance state) defines an operating point for the processor. States are named numerically starting from $\mathrm{P}_{0}$ to $\mathrm{P}_{\mathrm{N}}$, with $\mathrm{P}_{0}$ representing the maximum performance level. As the p-state number increases, the performance and power consumption of the processor decrease. Table 3.7 shows p-state definitions for a typical processor. The state definitions are made by the processor designer and represent a range of performance levels that match expected performance demand of actual workloads. Pstates are simply an implementation of dynamic voltage and frequency scaling. The resultant power reduction is obtained using these states is largely dependent on the amount of voltage reduction attained in the lower frequency states.

Table 3.7 Example P-states Definition

\begin{tabular}{|l|l|l|}
\hline P-State & Frequency $(\mathrm{MHz})$ & VDD (Volts) \\
\hline $\mathrm{P}_{0}$ & $\mathrm{~F}_{\mathrm{Max}} \times 100 \%$ & $\mathrm{~V}_{\mathrm{Max}} \times 100 \%$ \\
\hline $\mathrm{P}_{1}$ & $\mathrm{~F}_{\mathrm{Max}} \times 85 \%$ & $\mathrm{~V}_{\mathrm{Max}} \times 96 \%$ \\
\hline $\mathrm{P}_{2}$ & $\mathrm{~F}_{\mathrm{Max}} \times 75 \%$ & $\mathrm{~V}_{\mathrm{Max}} \times 90 \%$ \\
\hline $\mathrm{P}_{3}$ & $\mathrm{~F}_{\mathrm{Max}} \times 65 \%$ & $\mathrm{~V}_{\mathrm{Max}} \times 85 \%$ \\
\hline $\mathrm{P}_{4}$ & $\mathrm{~F}_{\mathrm{Max}} \times 50 \%$ & $\mathrm{~V}_{\mathrm{Max}} \times 80 \%$ \\
\hline
\end{tabular}


Table 3.8 Example C-states Definition

\begin{tabular}{|c|c|c|}
\hline C-State & Response Latency(us) & Relative Power \\
\hline $\mathrm{C}_{0}$ & 0 & $100 \%$ \\
\hline $\mathrm{C}_{1}$ & 10 & $30 \%$ \\
\hline $\mathrm{C}_{2}$ & 100 & $5 \%$ \\
\hline
\end{tabular}

\subsubsection{Idle Power Management: C-states}

A c-state (CPU idle state) defines an idle operating point for the processor. States are named numerically starting from $\mathrm{C}_{0}$ to $\mathrm{C}_{\mathrm{N}}$, with $\mathrm{C}_{0}$ representing the active state. As the c-state number increases, the performance and power consumption of the processor decrease. Table 3.8 shows c-state definitions for a typical processor. Actual implementation of the c-state is determined by the designer. Techniques could include low latency techniques, clock and fetch gating, or more aggressive high latency techniques such as voltage scaling or power gating.

\subsubsection{Case Study: Processor Power Management Characteristics}

The power saving states described in this section provides a significant range of power and performance settings for optimizing efficiency, limiting peak power consumption, or both. However, other parameters greatly influence the effective power consumption. Temperature, workload phase behavior, and power management policies are the dominant characteristics. Temperature has the greatest effect on static leakage power. This can be seen in Figure 3.2 which shows power consumption of a synthetic workload at various combinations of temperature and frequency. Note that ambient temperature is $20^{\circ} \mathrm{C}$ and "idle" temperature is $35^{\circ} \mathrm{C}$. 


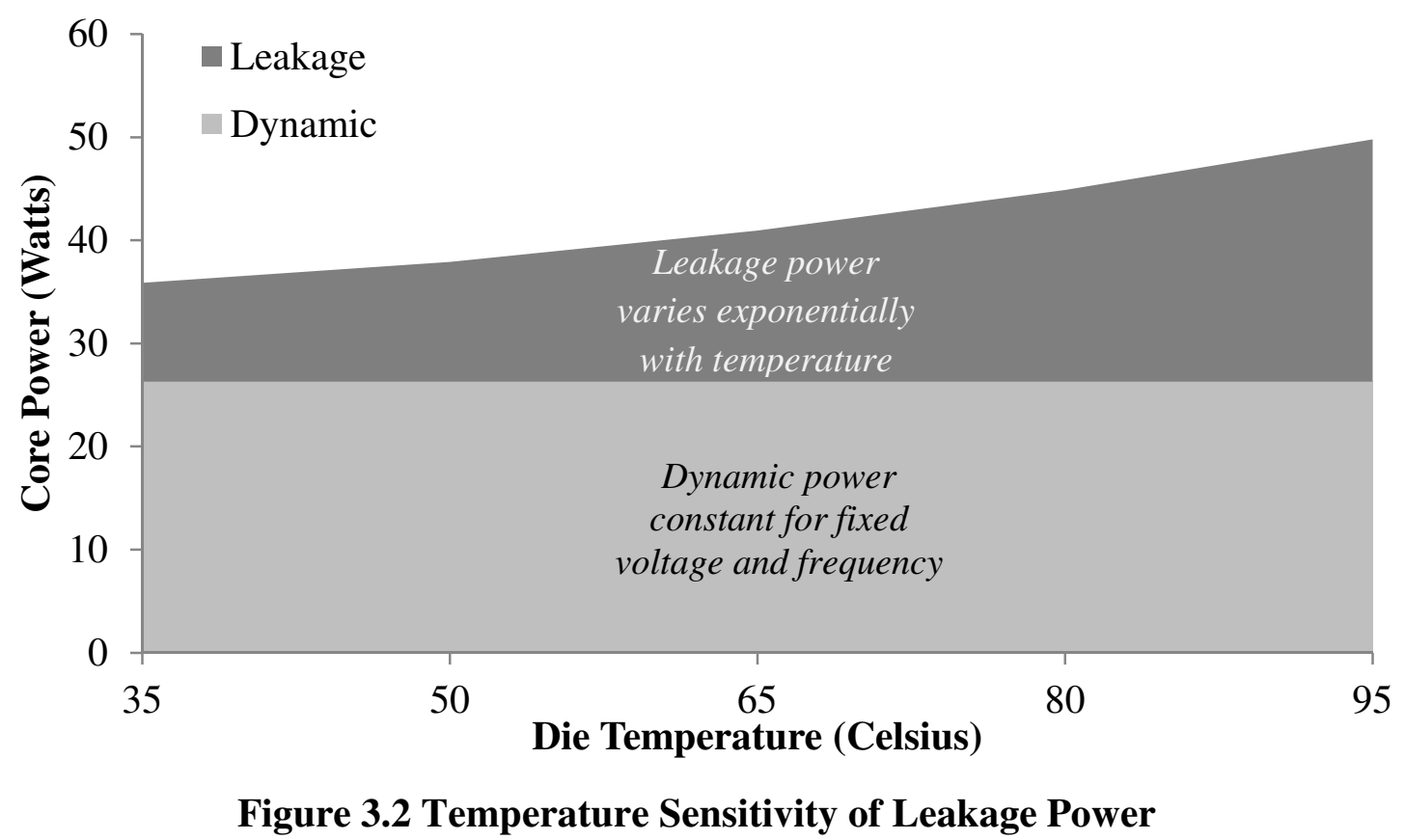

As expected, a linear change in frequency yields a linear change in power consumption. However, linear changes in temperature yield exponential changes in power consumption. Note that static power is identified by the Y-intercept in the chart. This is a critical observation since static power consumption represents a large portion of total power at high temperatures. Therefore, an effective power management scheme must also scale voltage to reduce the significant leakage component. To see the effect of voltage scaling consider Figure 3.3.

Figure 3.3 shows the cumulative effect of p-states and c-states. Combinations of five pstates (x-axis) and four operating modes are shown. The lowest power case, $\mathrm{C}_{1 \mathrm{e}}$-Idle, represents all cores being idle for long enough that the processor remains in the $\mathrm{C}_{1 \mathrm{e}}$ state more than 90 percent of the time. The actual amount of time spent in this state is heavily 
influenced by the rate of input/output (I/O) and OS interrupts. This state also provides nearly all of the static power savings of the low-voltage p-states even when in the $\mathrm{P}_{0}$ state. Second, the $\mathrm{C}_{1}$-Idle case shows the power consumption assuming at least one core remains active and prevents the processor from entering the $\mathrm{C}_{1 \mathrm{e}}$ state. This represents an extreme case in which the system would be virtually idle, but frequent interrupt traffic prevents all cores from being idle. This observation is important as it suggests system and OS design can have a significant impact on power consumption. The remaining two cases, $\mathrm{C}_{0}$-Idle and $\mathrm{C}_{0}$-Max, show the impact of workload characteristics on power. $\mathrm{C}_{0^{-}}$ Idle attains power savings though fine-grain clock gating.

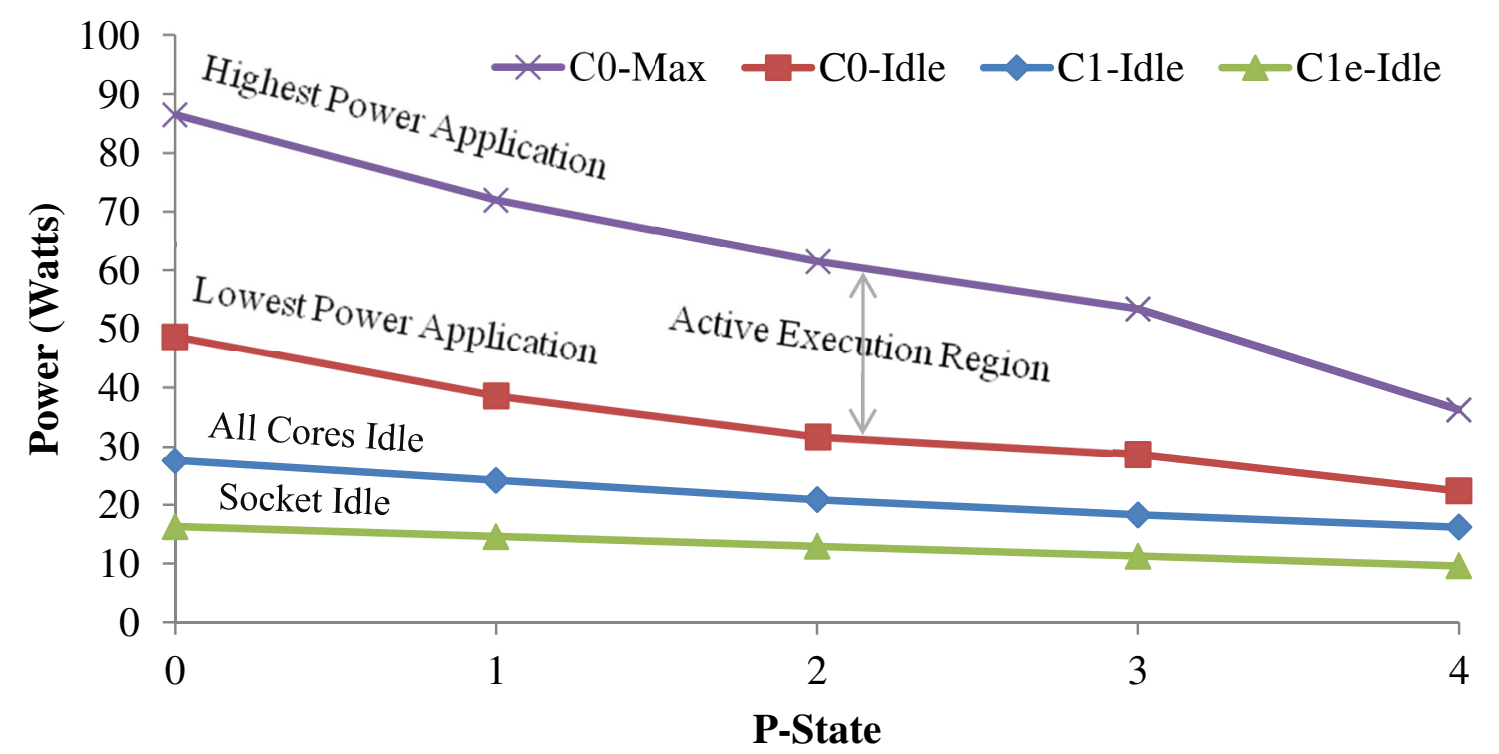

\begin{tabular}{|l|l|}
\hline $\mathrm{C}_{0}$-Max & All Cores Active IPC $\approx 3$ \\
\hline $\mathrm{C}_{0}$-Idle & All Cores Active IPC $\approx 0$ \\
\hline $\mathrm{C}_{1}$ - Idle & At Least One Active Core, Idle Core Clocks Gated \\
\hline $\mathrm{C}_{1 \mathrm{e}}$-Idle & "Package Idle" - All Core Clocks Gated, Memory Controller Clocks Gated \\
\hline
\end{tabular}

Figure 3.3 Power by C-state/P-state Combination 
The difference between $\mathrm{C}_{0}$-Idle and $\mathrm{C}_{0}$-Max is determined by the amount of power spent in switching transistors, which would otherwise be clock-gated, combined with worstcase switching due to data dependencies. $\mathrm{C}_{0}$-Max can be thought of as a pathological workload in which all functional units on all cores are 100 percent utilized and the datapath constantly switches between 0 and 1 . All active phases of real workloads exist somewhere between these two curves. High-IPC compute-bound workloads are closer to $\mathrm{C}_{0}$-Max while low-IPC memory-bound workloads are near $\mathrm{C}_{0}$-Idle.

\subsubsection{Power Management-Aware Model}

The model improves on existing on-line models [Be00] [BiJo06-1] [IsMa03] by accounting for power management and temperature effects. Like existing models it contains a workload dependent portion that is dominated by the number of instructions completed per second. In this case the number of fetched operations per second is used in lieu of instructions completed. The fetched $\mu$ ops metric is preferred as it also accounts for speculative execution. In addition to fetched $\mu$ ops, a retired floating point $\mu$ ops metric is also included. This accounts for the power difference between integer and floating point ops in the AMD processor. Unlike the Pentium 4 processor which exhibits little difference in power consumption between integer and floating point applications, the AMD processor exhibits much higher power consumption for high-throughput floating point applications. A further distinction of this model is that it contains a temperature dependent portion. Using workloads with constant utilization, processor temperature and voltage are varied to observe the impact on static leakage power. 
Temperature is controlled by adjusting the speed of the processor's fan. Temperature is observed with a $1 / 8$ degree Celsius resolution using an on-die temperature sensor [Bk09]. This sensor can be accessed by the system under test through a built-in, on-chip register. The resultant temperature-dependent leakage equation is shown in Table 3.9. Since temperature is modeled over only the operating range of the processor, it can be accounted for as a quadratic equation. Alternatively, a wider temperature range can be accounted for using and an exponential equation in the form of axe $\mathrm{e}^{\mathrm{T} \times \mathrm{b}}$. The coefficients $a$ and $b$ are found through regression. The term $\mathrm{T}$ represents the die temperature in Celsius. For this study the quadratic form is used due to its lower computational overhead and sufficient accuracy. Voltage is controlled using the P-State Control Register [Bk09]. This allows selection of one of five available voltage/frequency combinations. Voltage is observed externally as a subset of the traced power data. Like the workload dependent model, the coefficients of the static power model are tuned using regression techniques. Note that the static power model is highly process dependent. Processors with different semiconductor process parameters require the model to be re-tuned.

The dominant power management effects (voltage/frequency scaling, clock gating) are further accounted for using the gateable and ungateable power models. Gateable power is found by measuring the effect of enabling/disabling idle core clock gating. Ungateable represents the portion of power which cannot be gated. These components are also found experimentally. The resultant, average error in the model is $0.89 \%$. The standard deviation of the error for SPEC CPU2006 and SYSmark 2007 is less than 1\%. Worst- 
case error is $3.3 \%$. Alternatively stated, $68.2 \%$ of workloads have an error of less than 1\%. Per workload error is shown in Figure 3.4. The composition of the CPU model is summarized in Table 3.9.

Table 3.9 AMD Quad-Core Power Model

\begin{tabular}{|c|c|}
\hline Power Models & Equation \\
\hline Total Power & $\begin{array}{c}\sum_{N=0}^{3}(\text { WorkloadDependentN }+ \text { UngateableN }+ \text { GateableN }) \\
+ \text { Static }{ }_{\text {Volt }, \text { Temp }}\end{array}$ \\
\hline $\begin{array}{l}\text { Workload } \\
\text { Dependent } \\
\text { Power }\end{array}$ & 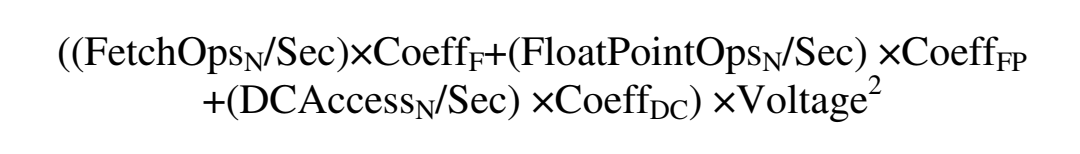 \\
\hline $\begin{array}{c}\text { Idle Power } \\
\text { Management } \\
\text { Power } \\
\text { (Gateable) }\end{array}$ & $\left(\%\right.$ Halted $\left._{N}\right) \times$ Coeff $_{\text {Gateable }} \times$ Voltage $^{2} \times$ Frequency $_{N}$ \\
\hline $\begin{array}{c}\text { Irreducible } \\
\text { Power } \\
\text { (Ungateable) }\end{array}$ & $\left(\%\right.$ NonHalted $\left._{N}\right) \times$ Coeff $_{\text {Ungateable }} \times$ Voltage $^{2} \times$ Frequency $_{N}$ \\
\hline $\begin{array}{c}\text { Irreducible } \\
\text { Power } \\
\text { (Static) }\end{array}$ & $\left(\operatorname{Temp}^{2} \times \operatorname{Coeff}_{\mathrm{T}}{ }^{2}+\operatorname{Temp}^{1} \times \operatorname{Coeff}_{\mathrm{T}}{ }^{1}+\times \operatorname{Coeff}_{\mathrm{T}}^{0}\right)_{\text {VoltageN }}$ \\
\hline
\end{tabular}

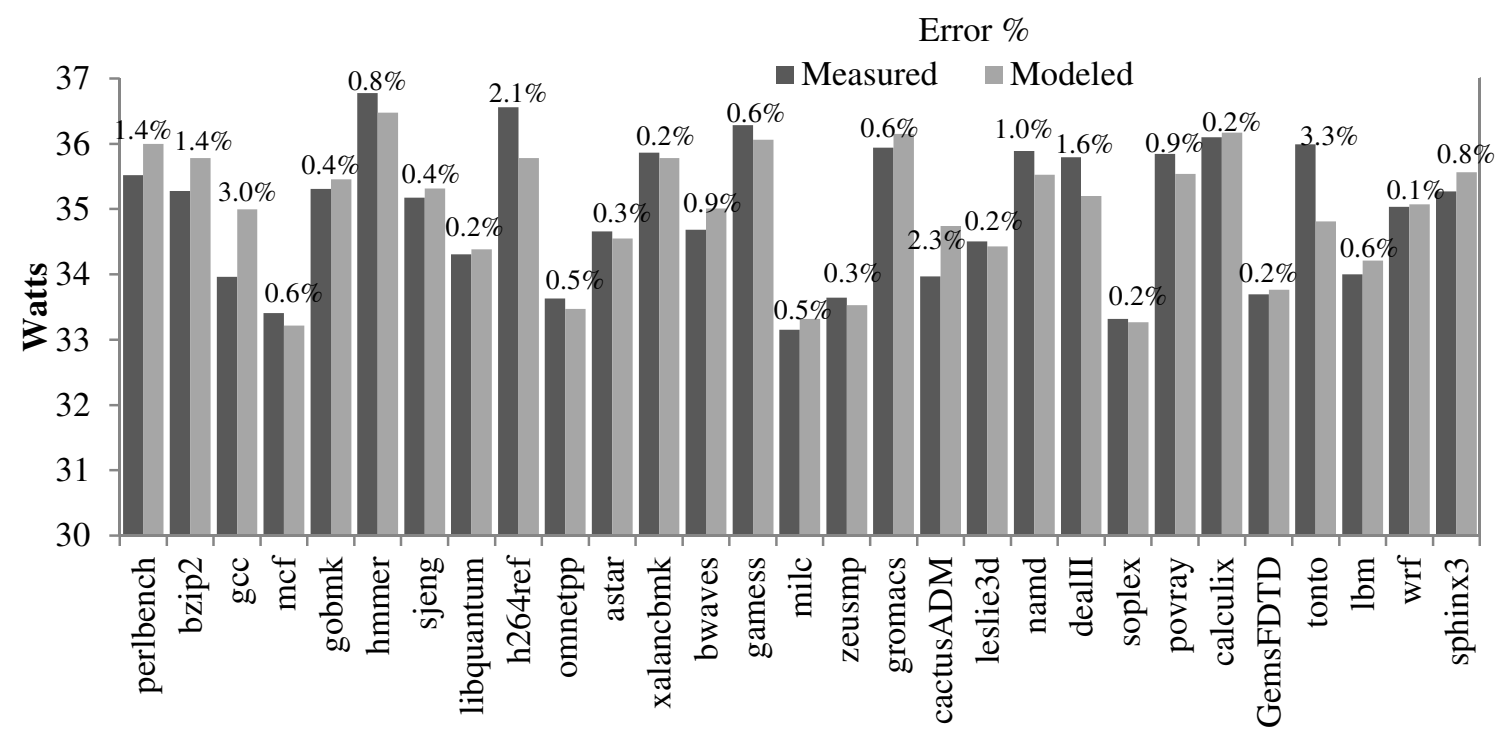

Figure 3.4 CPU Power Model - SPEC CPU 2006 Power and Average Error 


\subsection{Methodology for Power Modeling}

With an understanding of system level events that are visible to the processor it is possible to apply the iterative modeling process as depicted in Figure 3.5. This procedure utilizes linear and polynomial regression techniques to build power models for individual subsystems. The user identifies workloads which target a particular subsystem (cache, system memory, disk) and performs regression modeling using performance events as inputs. The model is then applied to a larger set of workloads to confirm accuracy and the lack of outlier cases. Depending on the outcome, the process is repeated with alternate performance events as inputs. Though an exhaustive search of performance events can be performed, a rapid solution is found when events are selected with high correlation to subsystem activity. Details of the modeling process in Figure 3.5 are listed below.

1. Measure subsystem-level power using subset of workloads. Begin with simple, easy-to-run workloads.

2. Confirm that Coefficient of Variation is greater than $\alpha$ for the chosen workload. The simplest workloads often do not generate sufficient power variation for model tuning. For example consider any of the cache-resident workloads in SPEC CPU 2000 which generate little or no activity in subsystems outside of the processor cores such as memory. Tuning the model based on these low-variation workloads may cause the process to include performance events that do not correlate well with power. 
3. Based on basic domain knowledge, choose performance events, measureable by performance counters that are most relevant to the subsystem in question. Choose counters that are expected to "trickle-down" to other subsystems. The pool of candidate performance counters may need to be expanded if sufficient accuracy is not achieved.

4. Using the selected performance counter events as the input variables and subsystem power as the output variable, perform linear regression modeling. For example, in the general linear equation $y=m x+b$, vary the coefficients $m$ and $b$ until the sum-of-squares error is minimized. Multiple linear or polynomial regression may be used in subsequent iterations of algorithm if sufficient accuracy is not obtained.

5. Using a subset of workloads calculate average error per sample. If less than $\rho \%$ error cannot be achieved, choose an a new performance event. Selection of $\rho$ is dictated by the required model accuracy and time required for solution. Setting $\rho$ to a low (restrictive) value may extend time to solution. It may also prevent the process from finding a solution.

6. Assess the representativeness of the model by manually comparing graphs of modeled versus measured power. This avoids the case in which statistical assessment cannot detect major errors such as those seen in Anscombe's Quartet [An73].

7. Using complete set of workloads calculate average error per sample. If less than $\delta$ $\%$ error cannot be achieved, choose a new performance event. Like $\rho, \delta$ is selected according the accuracy and time-to-solution requirements. 


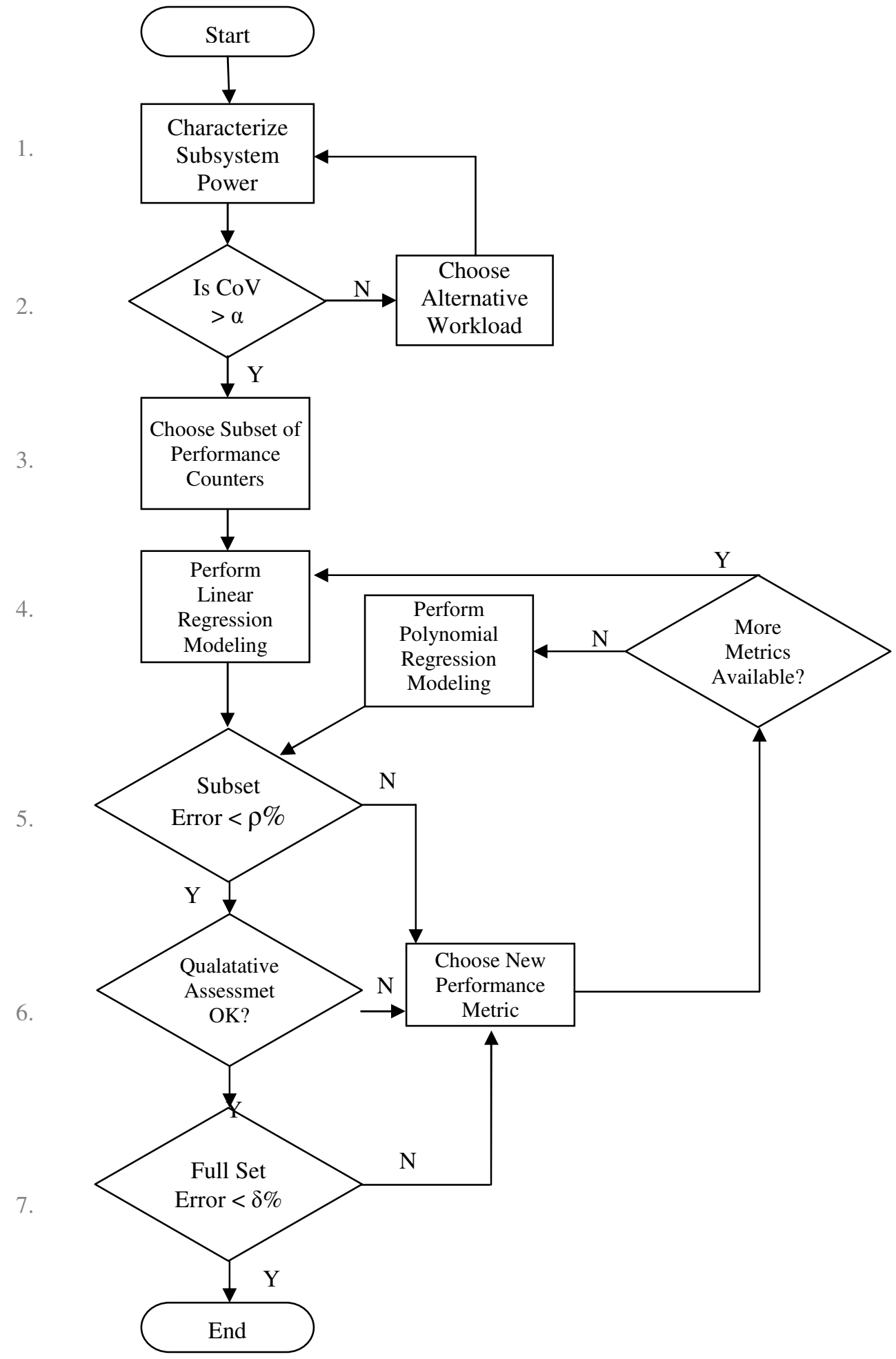

Figure 3.5 Trickle-Down Modeling Process 


\subsection{Summary}

This section describes concepts and methodologies for modeling processor power consumption through the use of performance monitoring counters. These models achieve average error rates of under $1 \%$ using only a handful of metrics/signals. Simple, deterministic, processor power models such as these will increase in importance as the need for energy efficiency increases. Techniques that maximize performance within fixed power limits or optimize power metrics (Perf/Watt, Energy $\times$ Delay, etc.) are becoming prevalent in processors [Po10][McPo06][ChJa09] and systems. Since these techniques rely on accurate processor power accounting, performance counter power models will increase in importance. 


\section{Chapter 4 System-Level Power Analysis}

This chapter provides an extensive characterization of system-level power consumption across platforms and workloads ranging from servers to laptops. For each system type, workloads and subsystems specific to the system are considered. Power consumption is considered in terms of average and variability. While average power is critical for energy efficiency, variation including maximum and minimum power is required for effective system and dynamic power management design.

\subsection{Average Power}

\subsubsection{Server Platform - SPEC CPU, SPECjbb and DBT-2}

For the case of SPEC CPU and SPECjbb workloads the behavior is distinct from the DBT-2 database workload. In Figure 4.1 a comparison of average subsystem power consumption is given for all workloads. Compared to the disk-bound DBT-2, the memory-bound and cpu-bound applications show significantly higher CPU and memory power consumption. While DBT-2 only increases average CPU power by $26 \%$ compared to idle, all of these workloads increase average CPU power by more than $250 \%$. For memory, the top three consumers are floating point workloads. This supports the intuitive conclusion that memory power consumption is correlated to utilization. 


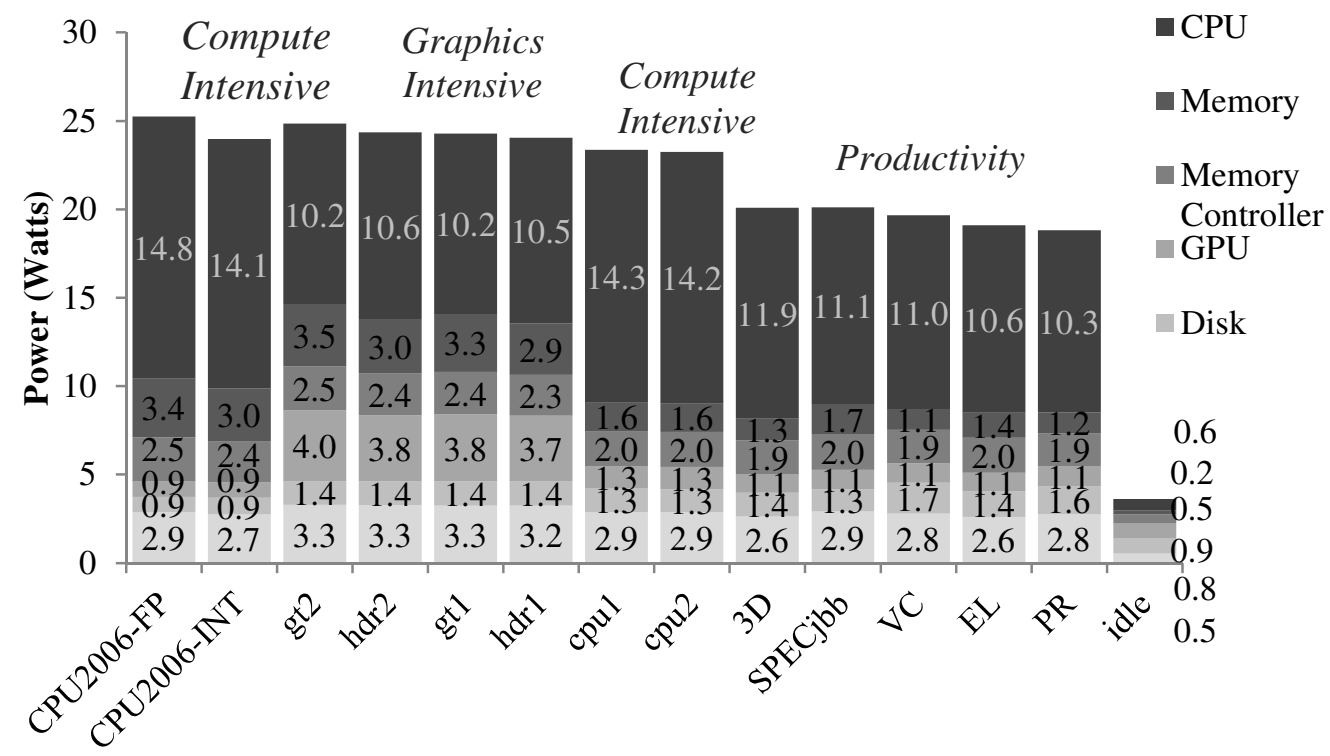

Figure 4.1 Average Power Consumption (Watts)

The remaining subsystems have little variation from workload to workload. For the disk subsystem this can be explained by two factors. First, most workloads used in this study contain little disk access with the exception of DBT-2. For most others, the initial loading of the working set is the majority of the disk access. Using synthetic workloads targeted at increasing disk utilization, less than a 3\% increase in average disk power can be achieved compared to idle. This is due to the second factor which is a lack of disk power management. Modern hard disks often have the ability to save power during low utilization through low power states and variable speed spindles. However, the server disks used in this study do not make use of these power saving modes. Therefore, disk power consumption is dominated by the power required for platter rotation which can account for almost $80 \%$ of max power [ZeSo03]. For I/O and chipset subsystems, little workload to workload variation is observable. In the case of the chipset, offset errors due 
to aliasing were introduced that affected average power results. Much greater variation can be found within each workload.

\subsubsection{SPEC CPU 2000/2006 CPU and Memory Power Comparison}

Compared to desktop and mobile systems servers have a different power consumption composition across subsystems. Due to the presence of large memory subsystems, DIMM power is a much larger component. Also, larger working sets such as those found in SPEC CPU2006 compared to SPEC CPU2000 shift power consumption from the cores to the DIMMs. Consider CPU2000 in Figure 4.2 and CPU2006 in Figure 4.3. Due to comparatively small working sets, CPU2000 workloads are able to achieve higher core power levels. The reason is that, since the working set fits completely within the on-chip caches, the processor is able to maintain high levels of utilization. This is made more evident by the power increases seen as the number of simultaneous threads is increased from one to four. Since there is less performance dependence on the memory interface, utilization and power continue to increase as threads are added. The result is different for SPEC CPU2006. Due to the increased working set size of this benchmark, the memory subsystem limits performance. Therefore, core power is reduced significantly for the four-thread case. Differences for the single-thread case are much less, due to a reduced dependency on the memory subsystem. The shift in utilization from the core to the memory subsystem can be seen clearly in Figure 4.4. For the most compute-bound workloads, core power is five times larger than DIMM power. However, as the 
workloads become more memory-bound, the power levels converge to the point where DIMM power slightly exceeds core power.

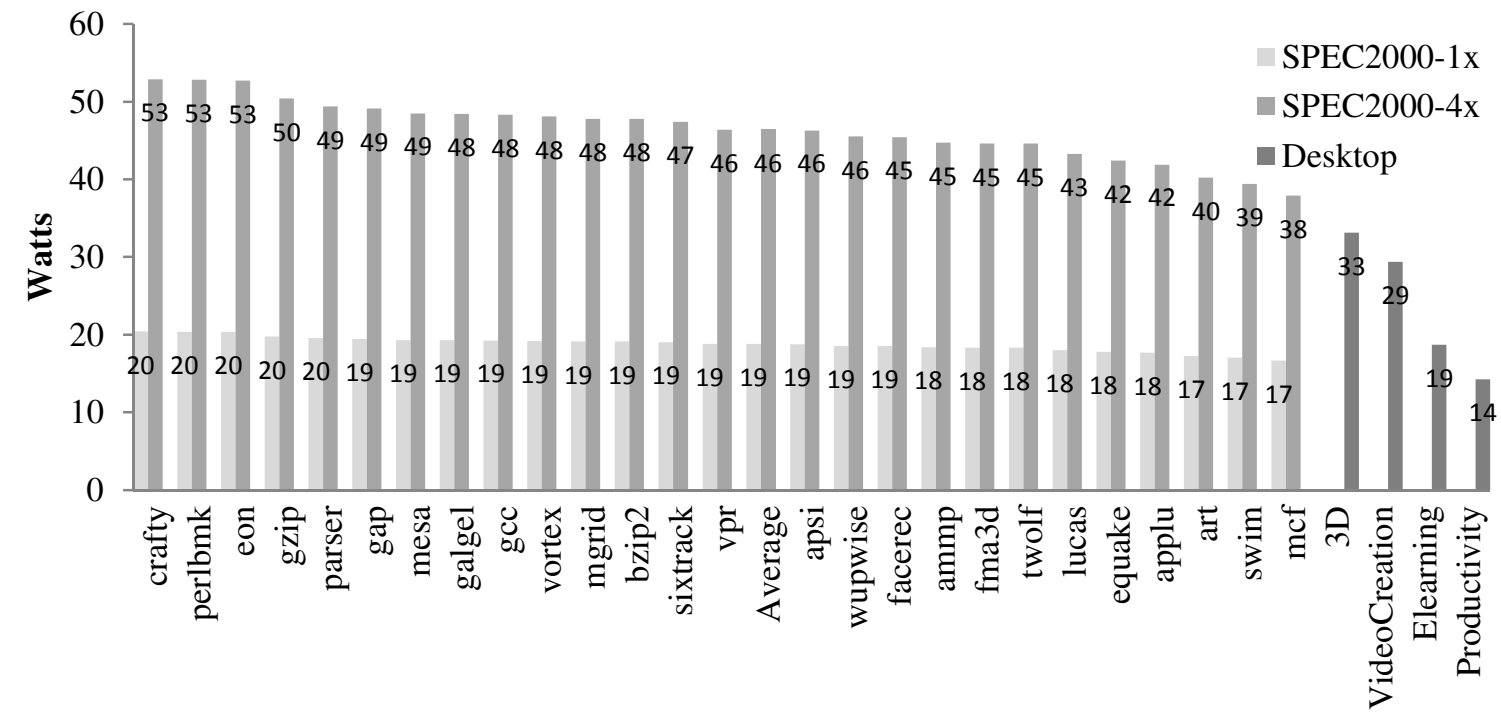

Figure 4.2 CPU2000 Average Core Power - 1 Thread vs. 4 Thread

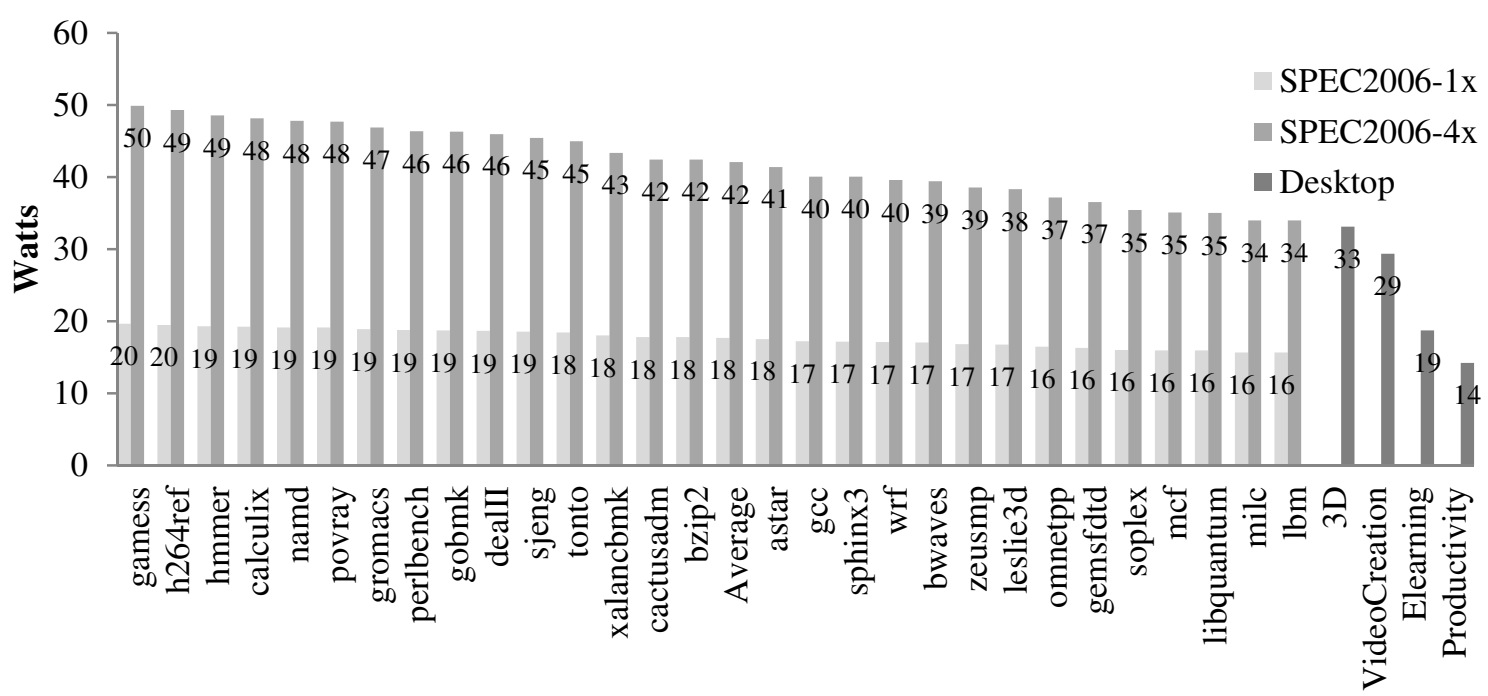

Figure 4.3 CPU2006 Average Core Power - 1 Thread vs. 4 Thread 


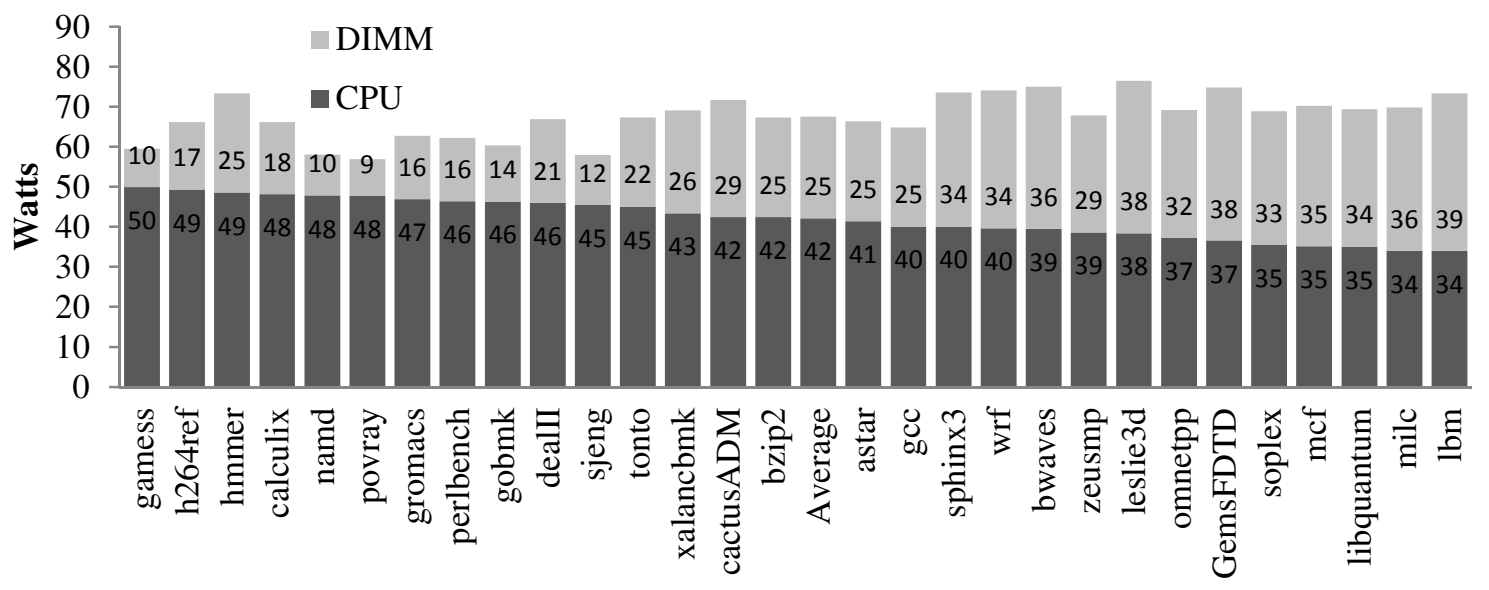

Figure 4.4 SPEC CPU2006 Average Core vs. DIMM Power

\subsubsection{Desktop Platform - SYSmark 2007}

In this section average power consumption levels across a range of workloads are considered. Two major conclusions for desktop workloads are drawn: the core is the largest power consumer and it contains the most variability across workloads. Though other subsystems, such as memory controller and DIMM, have significant variability within workloads, only the core demonstrates significant variability in average power across desktop workloads. Consider Figure 4.5: while average core power varies by as much as 57 percent, the next most variable subsystem, DIMM, varies by only 17 percent. Note, this conclusion does not hold for server systems and workloads in which much larger installations of memory modules cause greater variability in power consumption. The cause of this core power variation can be attributed to a combination of variable levels of thread-level parallelism and core-level power adaptations. In the case of 3D, the workload is able to consistently utilize multiple cores. 
At the other extreme, the productivity workload rarely utilizes more than a single core. Since Quad-Core AMD processor power adaptations are applied at the core level, frequency reduction achieves significant power savings on the three idle cores. As a result, the productivity workload consumes much less power than the $3 \mathrm{D}$ workload. The remaining workloads offer intermediate levels of thread-level parallelism and therefore have intermediate levels of power consumption. Also note that this level of power reduction is due only to frequency scaling. With the addition of core-level voltage scaling, the variation/power savings is expected to increase considerably.

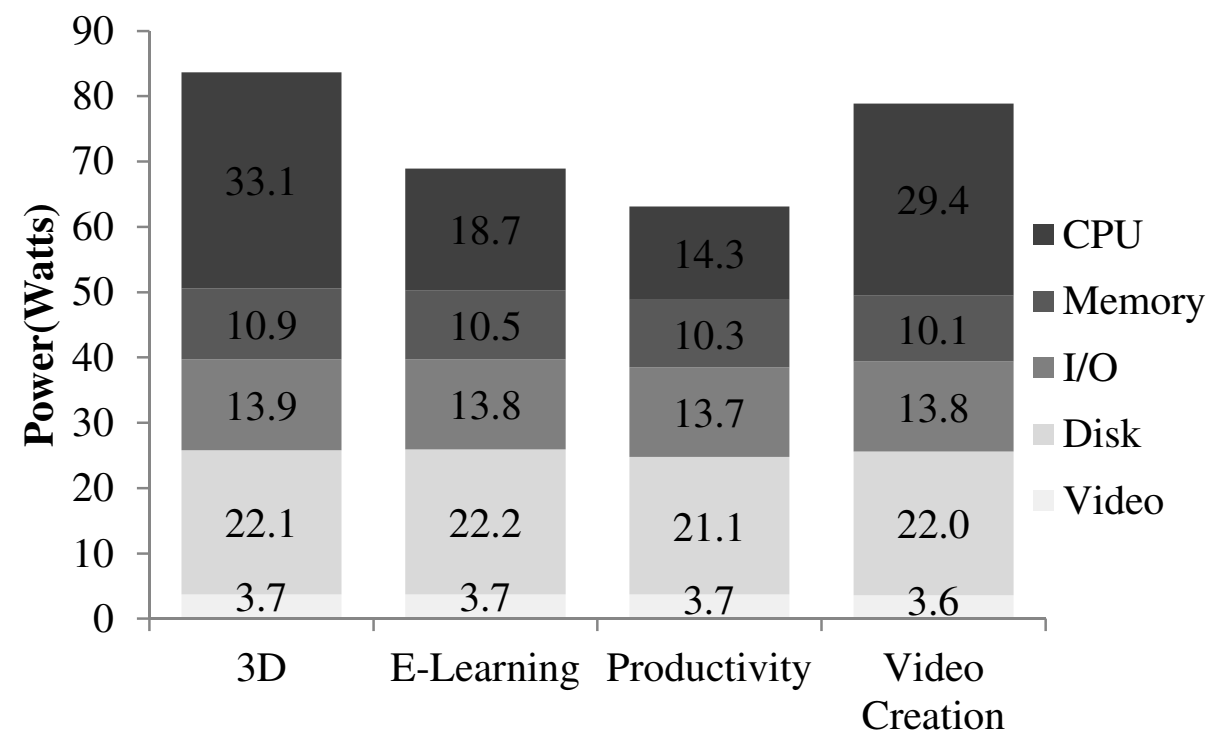

Figure 4.5 Desktop Subsystem Power Breakdown

\subsubsection{Desktop Platform - SPEC CPU, 3DMark and SYSmark}

To understand subsystem-level power consumption average and standard deviation results are presented. Figure 4.6 displays average power of each subsystem measured in Watts. To give an indication of the variation in power consumption Table 4.1 displays 
the standard deviation of subsystem power. Two major differences are apparent comparing desktop to server power consumption: desktop power in each subsystem is much less while relative variability is much greater. In both cases, power management plays a large role. Effective power management through DVFS, clock gating and link management reduce average power during idle and low utilization phases. This leads to a greater difference in sample-to-sample power. Additionally, semiconductor process improvements have a major effect.

The CPU subsystem is considered first. Not surprisingly, the desktop processor has average power that is an order of magnitude less than the server processor. This is largely influenced by process (130nM vs. 45nM), DVFS (desktop-only) and idle power management. While the server idle power represents at least $24 \%$ of average power, desktop idle power is no more than $4 \%$. These large power savings require the CPU model to include additional metrics such as frequency, voltage and temperature. It is not sufficient to consider metrics associated only with the instruction stream (IPC, cache accesses).

Like CPU, the chipset also exhibits much greater power variation. Unlike the server chipset which has nearly uniform power consumption, the desktop chipset has much greater variation with standard deviation representing as much as $10 \%$ of average power. The difference illustrates the impact of link (Hypertransport) power management. Average power values are also much less due to the lack of an L3 cache in the desktop 
processor. In both platforms the top-level cache is contained in the chipset power rail. To reduce power consumption and cost the desktop designer lacks an L3 cache.

Yet another subsystem with order-of-magnitude power reduction is the DRAM memory. Despite higher operating frequency $(533 \mathrm{Mhz}$ vs $100 \mathrm{MHz}$ ) average DRAM power is reduced by almost a factor of 10 . The reason is reduced memory voltage ( $2.8 \mathrm{~V}$ vs $1.5 \mathrm{~V})$, reduced capacity (8GB vs $4 \mathrm{~GB}$ ) and more aggressive memory power management. Note that the desktop system differentiates between DRAM, "Memory" subsystem and the Memory Controller. The server system includes both in the memory subsystem. The desktop memory controller has a similar level of power variation as the DRAMs. This is due to the memory controller management power savings for both subsystems. This also allows implementation of simple trickle-down models in multiple subsystems that are driven by the same performance metrics.

An additional subsystem, not present in the server analysis is the RS780 GPU (graphics processing unit). This subsystem has unique bi-modal power consumption. In all cases GPU power is either near the maximum or minimum levels. For workloads with little or no GPU activity power ranged from $0.8 \mathrm{~W}$ to $1.3 \mathrm{~W}$ with little variation. The graphicscentric workloads of 3DMark06 had much greater variation as the workload alternates between $\sim 1 \mathrm{~W}$ and $4 \mathrm{~W}$. This gives the GPU one of the largest power variations with a standard deviation covering over $25 \%$ of the maximum power. The bimodal power consumption is caused by aggressive idle power management and low active power variation. 


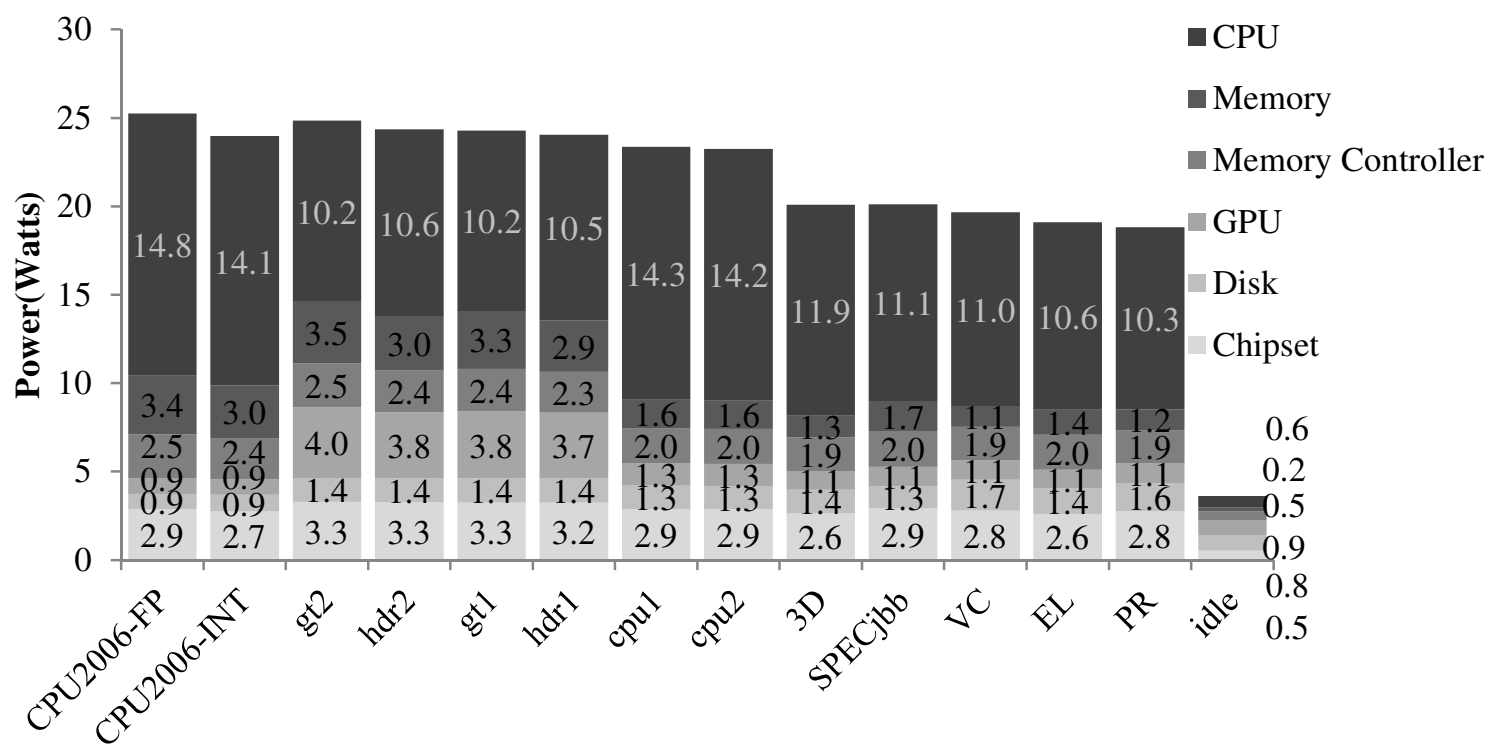

Figure 4.6 Subsystem Average Power (Watts)

Lastly the desktop hard drive power is considered. Three factors affect the large average power reduction and relative standard deviation increase: spindle speed, platter size and link power management. Since spindle power is such a large component of drive power consumption, reducing from 7200rpm to 5400rpm has a large impact. To conform to a smaller form factor, disk platter diameter is reduced nearly $28 \%$ (3.5" to 2.5 "). This reduces spindle and drive head power. Less power is required to rotate a smaller mass and move the drive head a shorter distance. Also, SATA link power management reduces power consumption in the control electronics and links during idle phases. These changes yield a drastic increase in variability with standard deviation representing $32 \%$ of average power in the most intense workload (video creation). 
Table 4.1 Subsystem Power Standard Deviation (Watts)

\begin{tabular}{|l|c|c|c|c|c|c|c|}
\hline \multicolumn{1}{|c|}{ Workload } & CPU & Chipset & Memory & $\begin{array}{c}\text { Memory } \\
\text { Controller }\end{array}$ & GPU & Disk & Total \\
\hline idle & 0.026 & 0.010 & 0.008 & 0.008 & 0.002 & 0.115 & 0.129 \\
\hline SPEC CPU2006 INT & 2.586 & 0.257 & 1.518 & 0.447 & 0.174 & 0.361 & 2.689 \\
\hline SPEC CPU2006 FP & 2.334 & 0.246 & 1.970 & 0.500 & 0.143 & 0.240 & 2.263 \\
\hline gt1 & 0.736 & 0.093 & 0.808 & 0.217 & 0.904 & 0.487 & 1.57 \\
\hline gt2 & 0.820 & 0.105 & 0.905 & 0.241 & 1.090 & 0.488 & 2.05 \\
\hline cpu1 & 1.989 & 0.262 & 0.706 & 0.168 & 0.356 & 0.469 & 2.02 \\
\hline cpu2 & 2.036 & 0.263 & 0.709 & 0.167 & 0.362 & 0.421 & 2.23 \\
\hline hdr1 & 0.757 & 0.131 & 1.043 & 0.294 & 1.144 & 0.527 & 1.84 \\
\hline hdr2 & 0.826 & 0.152 & 1.134 & 0.326 & 1.096 & 0.497 & 2.16 \\
\hline EL & 0.696 & 0.158 & 0.980 & 0.278 & 0.051 & 0.373 & 1.744 \\
\hline VC & 1.774 & 0.252 & 0.585 & 0.114 & 0.069 & 0.566 & 2.540 \\
\hline PR & 0.683 & 0.250 & 0.811 & 0.155 & 0.086 & 0.438 & 1.506 \\
\hline 3D & 1.159 & 0.170 & 0.587 & 0.108 & 0.029 & 0.321 & 1.701 \\
\hline SPECjbb & 1.230 & 0.297 & 1.096 & 0.235 & 0.031 & 0.232 & 2.765 \\
\hline
\end{tabular}

\subsection{Power Consumption Variation}

\subsubsection{Server Platform}

To quantify the extent of power variation within a workload coefficient of variation $(\mathrm{CoV})$ metric is used. This metric uses standard deviation to quantify variation in a data set, and also normalizes the variation to account for differences in average data. Since the subsystems in this study have average power values that differ by nearly an order of magnitude, this metric is most appropriate. Table 4.2 provides a summary of the coefficient of variation for all workloads.

Compared to the variation in average power among workloads on a given subsystem, the variation within a particular workload is less consistent. Subsystem-workload pairs such 
as CPU-gcc and memory-SPECjbb have a large variety of power levels. In contrast diskart and chipset-mcf have as much as $300 \mathrm{X}$ less variation.

Table 4.2 Coefficient of Variation

\begin{tabular}{|c|c|c|c|c|c|}
\hline & CPU & Chipset & Memory & I/O & Disk \\
\hline idle & $8.86 \times 10^{-3}$ & $4.61 \times 10^{-3}$ & $1.17 \times 10^{-3}$ & $3.86 \times 10^{-3}$ & $1.25 \times 10^{-3}$ \\
\hline gcc & $5.16 \times 10^{-2}$ & $1.13 \times 10^{-2}$ & $6.90 \times 10^{-2}$ & $4.05 \times 10^{-3}$ & $2.44 \times 10^{-3}$ \\
\hline mcf & $3.37 \times 10^{-2}$ & $8.53 \times 10^{-3}$ & $3.60 \times 10^{-2}$ & $3.81 \times 10^{-3}$ & $1.50 \times 10^{-3}$ \\
\hline vortex & $6.99 \times 10^{-3}$ & $4.12 \times 10^{-3}$ & $2.06 \times 10^{-2}$ & $3.11 \times 10^{-3}$ & $7.82 \times 10^{-4}$ \\
\hline art & $2.47 \times 10^{-3}$ & $3.66 \times 10^{-3}$ & $5.31 \times 10^{-3}$ & $3.12 \times 10^{-3}$ & $2.51 \times 10^{-4}$ \\
\hline lucas & $1.21 \times 10^{-2}$ & $6.34 \times 10^{-3}$ & $5.73 \times 10^{-3}$ & $3.09 \times 10^{-3}$ & $3.25 \times 10^{-4}$ \\
\hline mesa & $6.05 \times 10^{-3}$ & $3.49 \times 10^{-3}$ & $8.81 \times 10^{-3}$ & $3.86 \times 10^{-3}$ & $3.85 \times 10^{-4}$ \\
\hline mgrid & $3.58 \times 10^{-3}$ & $2.46 \times 10^{-3}$ & $3.36 \times 10^{-3}$ & $3.06 \times 10^{-3}$ & $2.37 \times 10^{-4}$ \\
\hline wupwise & $1.56 \times 10^{-2}$ & $6.96 \times 10^{-3}$ & $9.45 \times 10^{-3}$ & $3.12 \times 10^{-3}$ & $4.95 \times 10^{-4}$ \\
\hline DBT-2 & $1.70 \times 10^{-1}$ & $6.73 \times 10^{-3}$ & $2.37 \times 10^{-2}$ & $4.35 \times 10^{-3}$ & $1.61 \times 10^{-3}$ \\
\hline SPECjbb & $2.34 \times 10^{-1}$ & $1.75 \times 10^{-2}$ & $7.61 \times 10^{-2}$ & $1.70 \times 10^{-3}$ & $3.34 \times 10^{-3}$ \\
\hline
\end{tabular}

The cause for this difference can be attributed to the presence or lack of power management in the various subsystems. The most variable subsystem, the CPU, makes use of explicit clock gating through the instruction set. Whenever the operating system is unable to find a schedulable process, it issues the "halt" instruction. This puts the processor in a low power mode in which the clock signal is gated off in many parts of the chip. This mode reduces power consumption in the processor to less than $25 \%$ of typical. Since the memory subsystem does not make use of significant power management modes, its variation is due only to varying levels of utilization. Since these workloads exhibit large variations in memory utilization, this has a significant impact.

In contrast, the chipset and I/O subsystems have little variation in utilization. Since these subsystems also do not make use of power saving modes, their total variation is low. In 
the case of $\mathrm{I} / \mathrm{O}$, the observed workloads make little or no use of disk and network resources. For the chipset subsystem, the causes are not as clear and require further study. As mentioned in the previous section the lack of disk power management causes little variation in disk power consumption. If these subsystems are to benefit from dynamic adaptation, workloads with larger variation in utilization would be needed.

In order to justify the use of $\mathrm{CoV}$ for identifying workloads with distinct, adaptable phases the probability distributions for extreme cases are considered. In order for a subsystem-workload pair to be a strong candidate for optimization, it must have distinct program/power phases. If a workload exhibits constant power consumption it is difficult to identify distinct phases. Further if the difference in phases is small, it may be difficult to distinguish a phase in the presence of sampling noise. Therefore, a strong candidate should have multiple distinct phases. This can be observed in the power amplitude distributions in Figure 4.7. A narrow distribution represents a constant power consumption. A wide distribution represents many levels of power consumption. Distinct power phases only exist in the wide distributions.

Figure 4.7 shows the average power distribution for the various subsystems. Not surprisingly, CPUs are the dominant power users. However, unlike distributed, scientific NAS Parallel Benchmark [FeGe05-1] and mobile, productivity workloads such as PCMark and 3DMark [MaVa04], I/O and disk power are significant. While differences in average subsystem power are large at $138 \%$ for disk compared to CPU, the variations within an individual subsystem are even greater. A comparison of subsystem power 
amplitude distributions is made in Figure 4.7. Note that the CPU distribution is truncated at 60 Watts to prevent obscuring results from the other subsystems. A small number of phases $(6.5 \%)$ exist above 60 Watts and extending to 163 Watts.

These distributions suggest that there are significant opportunities for phase-based power savings for $\mathrm{CPU}, \mathrm{I} / \mathrm{O}$, and disk. These subsystems have more wider and/or multimodal distributions. The larger variations in power consumption provide greater opportunity to use runtime detection techniques such as [In06] [IsMa06]. In contrast, chipset and memory have homogeneous behavior suggesting nearly constant power consumption and less opportunity for phase detection.

The presence of power variation is not sufficient to motivate the application of power adaptation. Due to the overhead of detection and transition, adapting for short duration phases may not be worthwhile. Table 4.3 presents the percentage of samples that are classifiable as phases with durations of $1 \mathrm{~ms}, 10 \mathrm{~ms}, 100 \mathrm{~ms}$ and $1000 \mathrm{~ms}$. A group of power samples is considered a phase if the power level within the group remains constant. To quantify the similarity, the coefficient of variation $(\mathrm{CoV})$ is calculated for the group. The group is considered a phase if the CoV does not exceed a specified threshold. The boundaries of a phase are determined by samples which cause the CoV to exceed the threshold. Results for $\mathrm{CoV}$ of $0.25,0.1$ and 0.05 are presented. At thresholds of 0.25 and 0.1 excessive error exists especially in $\mathrm{I} / \mathrm{O}$ subsystem phase classifications. A probable cause of the error is the greater sample-to-sample variability of the I/O power trace. The disk subsystem, which has higher than average error, also has a wider than 
average distribution. The apparent increased residency for longer phases is specific to the high $\mathrm{CoV}$ cases. The reason is that by including a larger number of samples (longer phase length) in the $\mathrm{CoV}$ calculation and using a high $\mathrm{CoV}$ threshold, the "real" phase behavior is obscured. Actual phase edges get averaged out by the larger number of samples. This is primary reasons for choosing $\mathrm{CoV}=0.05$ for the subsequent analysis. It exhibits the desired behavior of distinguishing the long and short phases. For the following discussion, a $\mathrm{CoV}$ of 0.05 is utilized.

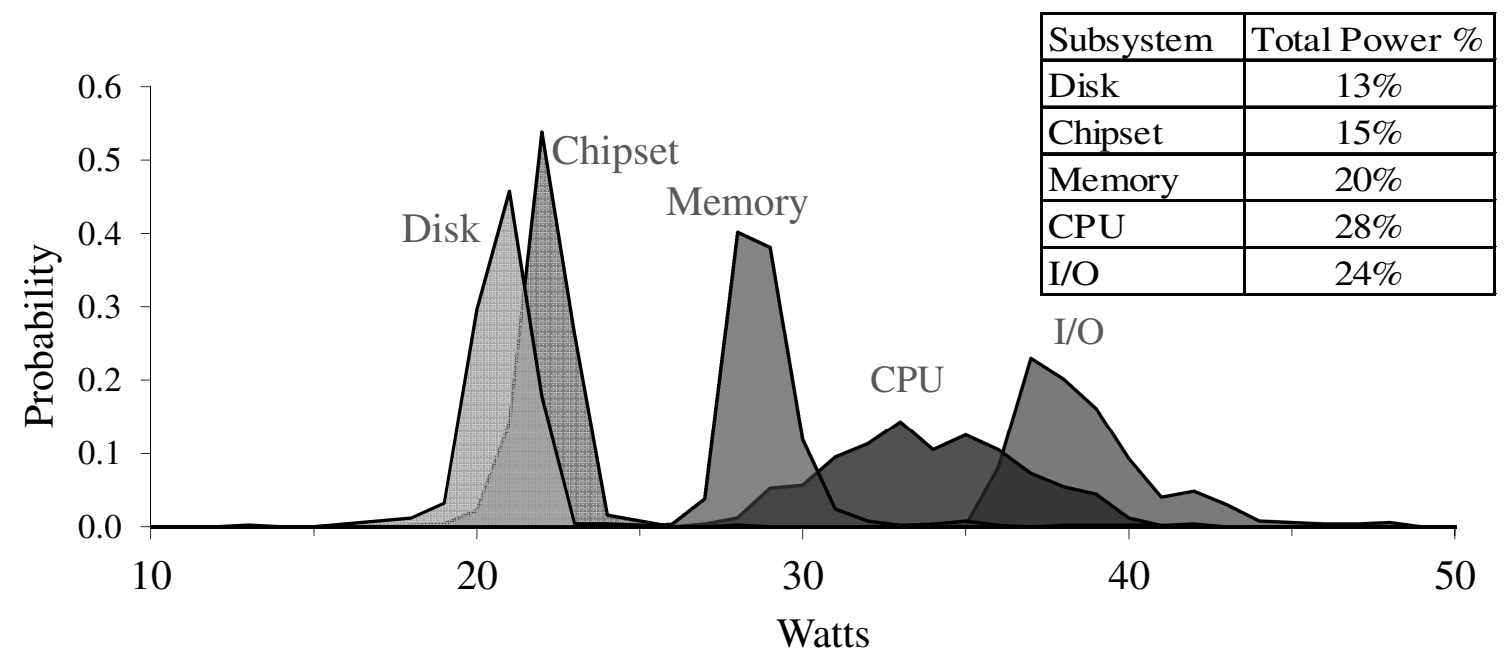

Figure 4.7 Subsystem Amplitude Distributions

The effect of narrow chipset and memory distributions is evident in their high rates of classification. For both, at least half of all samples can be classified as $1000 \mathrm{~ms}$ phases. In contrast, CPU, I/O and disk have no $1000 \mathrm{~ms}$ phases and considerably fewer phases classified at finer granularities. These results can be used to plan power management strategies for a particular workload. For example, by noting that the I/O subsystem has almost no phases longer than $1 \mathrm{~ms}$, the designer would be required to use low latency 
adaptations. In contrast, the disk subsystem has $18.5 \%$ of samples definable as $100 \mathrm{~ms}$ phases, thus providing greater opportunity to amortize adaptation costs. While chipset and memory subsystems have a large percentage of classifiable samples, they may not be viable candidates for adaptation. By also considering that most of the chipset and memory samples are close to the average, standard deviations of 0.9 Watts and 1.4 Watts respectively, there may be insufficient variation for runtime phase detection.

Table 4.3 Percent of Classifiable Samples

\begin{tabular}{|c|l|l|l|l|l|}
\hline Duration (ms) & CPU & Chipset & Memory & I/O & Disk \\
\hline \multicolumn{7}{|c|}{ CoV $=0.25$} \\
\hline 1 & 98.5 & 100 & 100 & 99.5 & 100 \\
\hline 10 & 90.8 & 100 & 100 & 87.6 & 100 \\
\hline 100 & 70.0 & 100 & 100 & 85.3 & 100 \\
\hline 1000 & 36.0 & 100 & 100 & 96.3 & 100 \\
\hline Error \% & 8.78 & 3.70 & 3.47 & 15.2 & 6.31 \\
\hline \multicolumn{7}{|c|}{ CoV $=0.10$} \\
\hline 1 & 91.7 & 100 & 100 & 81.1 & 100 \\
\hline 10 & 66.0 & 100 & 98.6 & 35.7 & 88.6 \\
\hline 100 & 43.1 & 100 & 94.4 & 21.0 & 95.6 \\
\hline 1000 & 9.30 & 100 & 93.1 & 0.00 & 95.0 \\
\hline Error \% & 4.60 & 3.70 & 3.47 & 6.63 & 6.31 \\
\hline \multicolumn{7}{|c|}{ CoV $=0.05$} & \\
\hline 1 & 61.6 & 88.3 & 97.7 & 22.4 & 98.4 \\
\hline 10 & 25.5 & 78.0 & 91.2 & 1.70 & 32.1 \\
\hline 100 & 6.00 & 63.2 & 78.6 & 0.00 & 18.5 \\
\hline 1000 & 0.00 & 64.4 & 50.0 & 0.00 & 0.00 \\
\hline Error \% & 3.38 & 3.46 & 2.68 & 3.67 & 2.93 \\
\hline
\end{tabular}

From these results, it is clear that distinct phases are detectable at granularities ranging from seconds to milliseconds. The next step in utilizing the phases is to combine the amplitude and duration results to direct power management strategies. An example classification is given in Table 4.4. 
Table 4.4 Workload Phase Classification

\begin{tabular}{|l|l|l|l|}
\hline & High Power & Med Power & Low Power \\
\hline High Duration & $5 \%$ & $10 \%$ & $20 \%$ \\
\hline Med Duration & $0 \%$ & $15 \%$ & $5 \%$ \\
\hline Low Duration & $10 \%$ & $35 \%$ & $0 \%$ \\
\hline
\end{tabular}

This classification can be used to direct selection of power saving techniques. The phase duration selects the power management type, based on similar transition times. The power level and frequency work in opposition to each other as a policy control. For example, a particular phase may only occur $5 \%$ of the time. However, since it is such a high power case it would be valuable to reduce its power. At the other extreme, a phase may consume low power, but since it occurs frequently it would be valuable to address.

\subsubsection{Desktop Platform}

In this section the intra-workload phase characteristics that contribute to the variation are considered. These results are attributable to the three dominant components of power adaptation: hardware adaptation, workload characteristics, and OS control of adaptations. In Figure 4.8 a distribution of the phase length of power consumption for desktop workloads is presented. Two major conclusions are drawn: the operating system has a significant effect on phase length and interactive workloads tend to have longer phases.

First, the two spikes at $10 \mathrm{~ms}$ and $100 \mathrm{~ms}$ show the effect of the operating system. These can be attributed to the periodic timer tick of the scheduler and p-state transitions requested by the operating system. In the case of Microsoft Windows Vista, the periodic timer tick arrives every 10-16 ms [Mm05]. This affects the observed power level since 
power consumed in the interrupt service routine is distinct from "normal" power levels. In the case of high-IPC threads, power is reduced while servicing the interrupt, which typically has a relatively low-IPC due to cold-start misses in the cache and branch predictor. In the case of low-power or idle threads, power is increased since the core must be brought out of one or more power saving states in order to service the interrupt. This is a significant problem for power adaptations since the timer tick is not workload dependent. Therefore, even a completely idle system must "wake up" every $10 \mathrm{~ms}$ to service an interrupt, even though no useful work is being completed. Also, $10 \mathrm{~ms}$ phase transitions are artificially introduced due to thread migration. Since thread scheduling is performed on timer tick intervals, context switches, active-to-idle, and idle-to-active transitions occur on $10 \mathrm{~ms}$ intervals. The $100 \mathrm{~ms}$ phases can be explained by the OS's application of p-state transitions. Experimentally, it can be shown that the minimum rate at which the operating system will request a transition from one p-state to another is 100 ms. When p-state transitions are eliminated, the spike at the $100 \mathrm{~ms}$ range of Figure 4.8 is eliminated.

The second conclusion from Figure 4.8 is that interactive workloads have longer phase durations. In the case of 3D and video creation workloads, a significant portion of time is spent in compute-intensive loops. Within these loops, little or no user interaction occurs. In contrast, the productivity and e-learning workloads spend a greater percentage of the time receiving and waiting for user input. This translates into relatively long idle phases which are evident in the lack of short duration phases in Figure 4.8. 


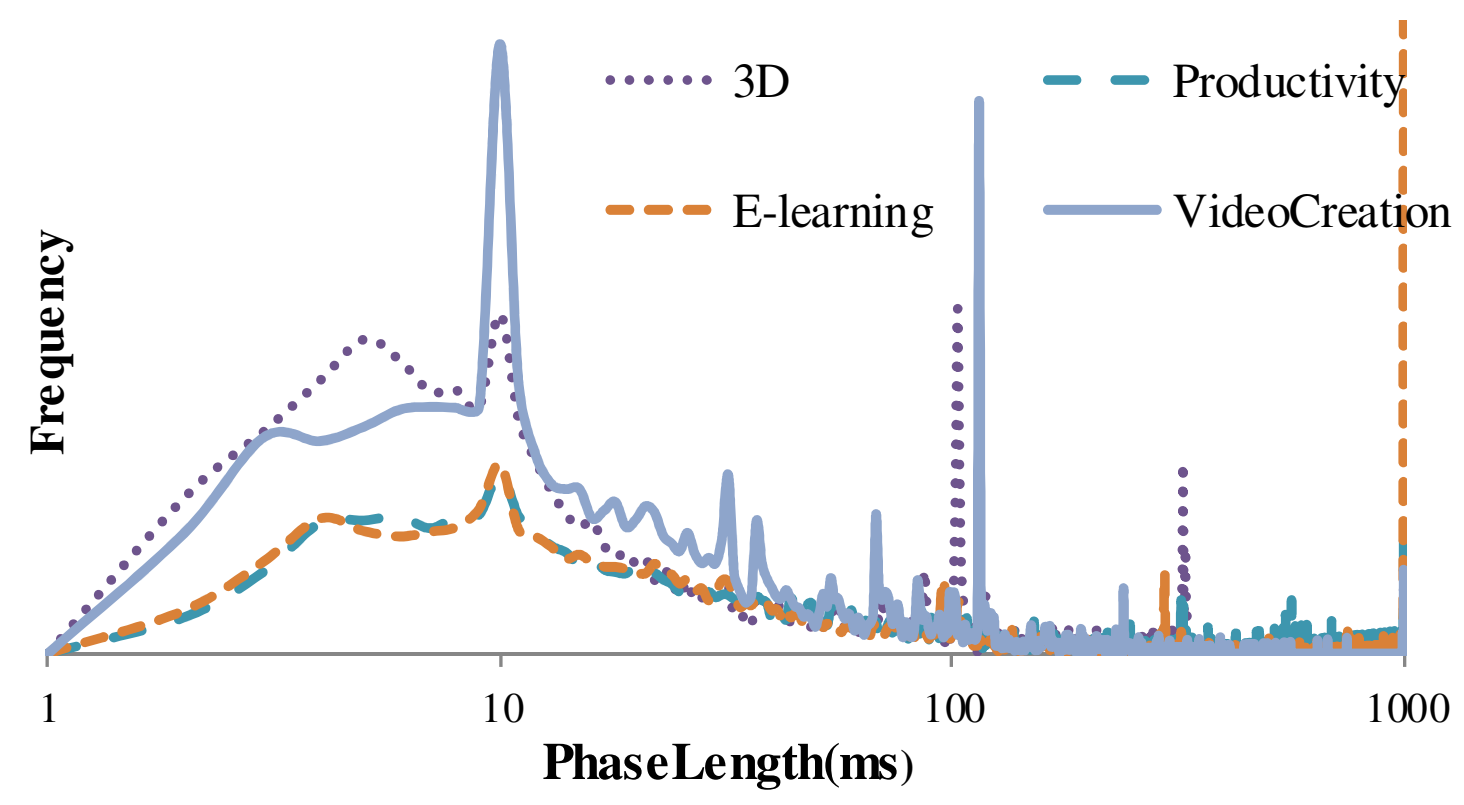

Figure 4.8 Core Power Phase Duration

This is further supported by Figure 4.9, which group the most common phases by combinations of amplitude and duration. Note that all phases less than $10 \mathrm{~ms}$ are considered to be $10 \mathrm{~ms}$. This simplifies presentation of results and is reasonable since the OS does not apply adaptation changes any faster than $10 \mathrm{~ms}$. These figures show that the highest power phases only endure for a short time. These phases, which are present only in $3 \mathrm{D}$ and - to a much lesser degree - in video creation, are only possible when multiple cores are active. The lack of long duration high power phases is attributable to two causes: low percent of multithreaded phases and higher IPC dependence during multithreaded phases. The dependence on IPC for phase length increases as the number of active cores increases. When many cores are active the power differences are caused by changes in active power due to IPC (performance). When few cores are active, total 
power is dominated by leakage power since active power is low. Leakage power is mostly affected by p-state changes. Fluctuations in IPC occur at much shorter durations (100s ns) than p-state changes (100s ms). Therefore, stable power consumption levels are less likely as the number of active cores increases.

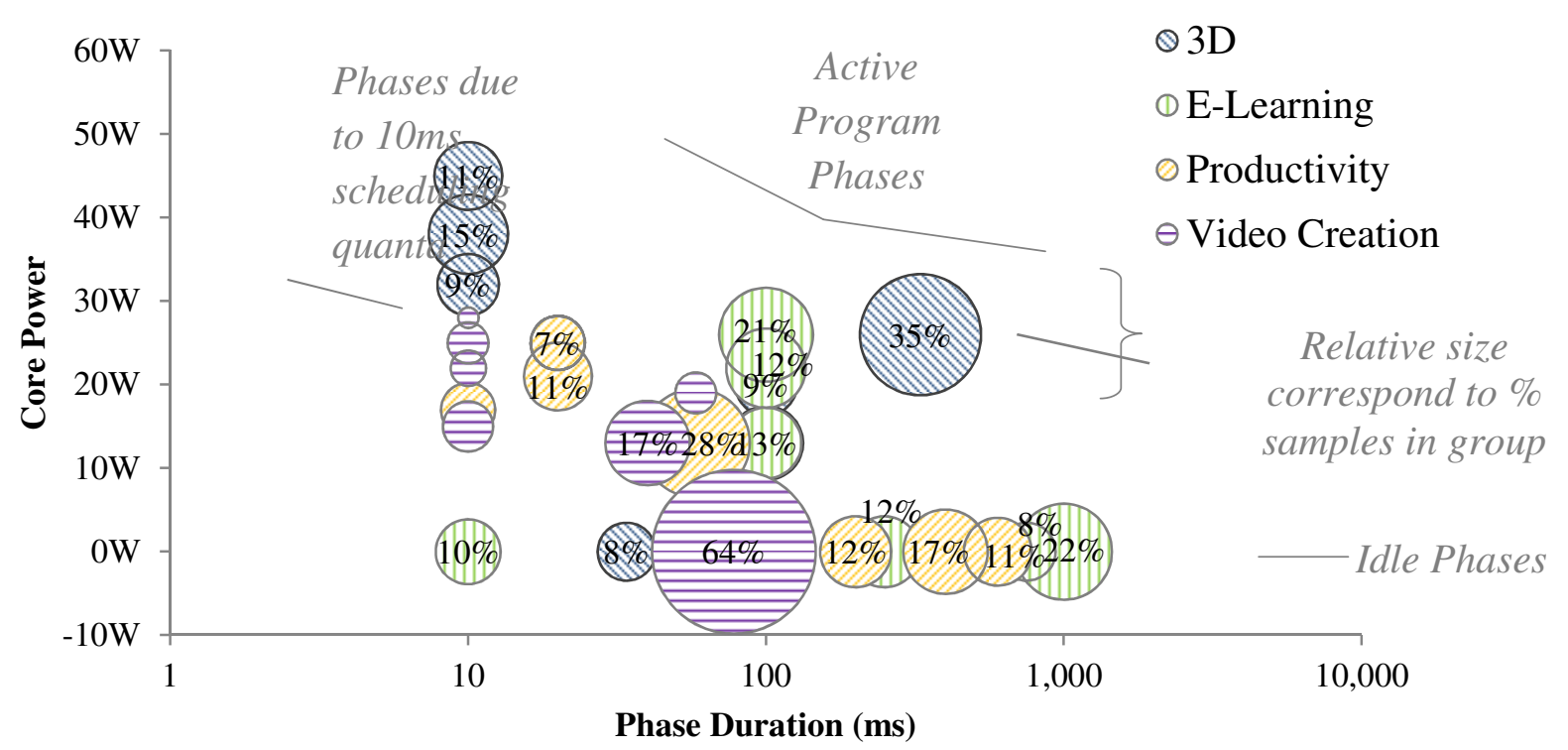

Figure 4.9 Core Power Phases - SYSmark 2007

\subsection{Summary}

This section characterizes power consumption in modern server and desktop computing systems. The characterization demonstrates the relationship between the power consumption of various subsystems and workloads. Popular computational workloads such as SPEC CPU are shown to generate power variation in the processor and memory subsystems, but not in the remainder of subsystems. By comparing power variation in a server system with little power management to a recent desktop system with extensive 
power management, it is shown that most variation is due to power management. This suggests that as systems employ more aggressive power management, instantaneous power consumption will increase its variability. Power limiting strategies will need to account for and respond to frequent power transitions due to the increased potential for performance loss or power overage. 


\section{Chapter 5 Modeling System-Level Power}

\section{using Trickle-Down Events}

This chapter presents system-level power models based on performance counter events within the processor. The first section defines the concept and intuition behind trickledown performance events. The second section describes the server power model. The last section describes the laptop power model.

\subsection{Processor Events Propagate to Rest of System}

Trickle-down power modeling provides an accurate representation of complete-system power consumption using a simple methodology. The approach relies on the broad visibility of system-level events to the processor. This allows accurate, performance counter based models to be created using only events local to the processor. These local events can be measured using ubiquitous performance counters found in all modern microprocessors. Local events are preferred since power models can be built using a single interface. There is no need to create interfaces to multiple devices and subsystems which have inconsistent or incomplete performance counter APIs (Application Programming Interface). It is particularly common at the system level since components are often designed by multiple vendors. Trickle-down modeling also addresses hardware costs in systems implementing direct measurement. Rather than providing sensors and 
power measurement hardware for multiple subsystems, measurement need only be implemented on a single system during the design stage. The model is created based on measurement from a small number of systems which allows power measurement hardware to be eliminated from the final product.

While the trickle-down approach simplifies power modeling of complete systems it requires a modest knowledge of subsystem level interaction. The effectiveness of the model at capturing system-level power is determined by the selection of comprehensive performance events. Some events such as top-level cache or memory accesses are intuitive. A miss in the first level cache will necessarily generate traffic in higher level caches and or the memory subsystem. Other events such as those found in I/O devices are not as obvious. Consider the system diagram in Figure 5.1.

This represents the quad-socket server for which the trickle-down modeling approach is applied. The arrows flowing outward from the processor represent events that originate in the processor and trickle-down to other subsystems (L3 Miss, TLB Miss, MemBus Access and Uncacheable Access). Arrows flowing inward such as DMA (Direct Memory Access) or bus master access and I/O interrupts may not be directly generated by the processor, but are nevertheless visible. Since DMA access is typically performed to addresses marked as cacheable by the processor, they can be observed in the standard cache access metrics. To distinguish DMA accesses by a particular device, events should be qualified by address range. Each device typically uses a private range of addresses in 
system memory for DMA access. Similarly interrupts from multiple devices can be distinguished by interrupt number or address in the case of message signaled interrupts.

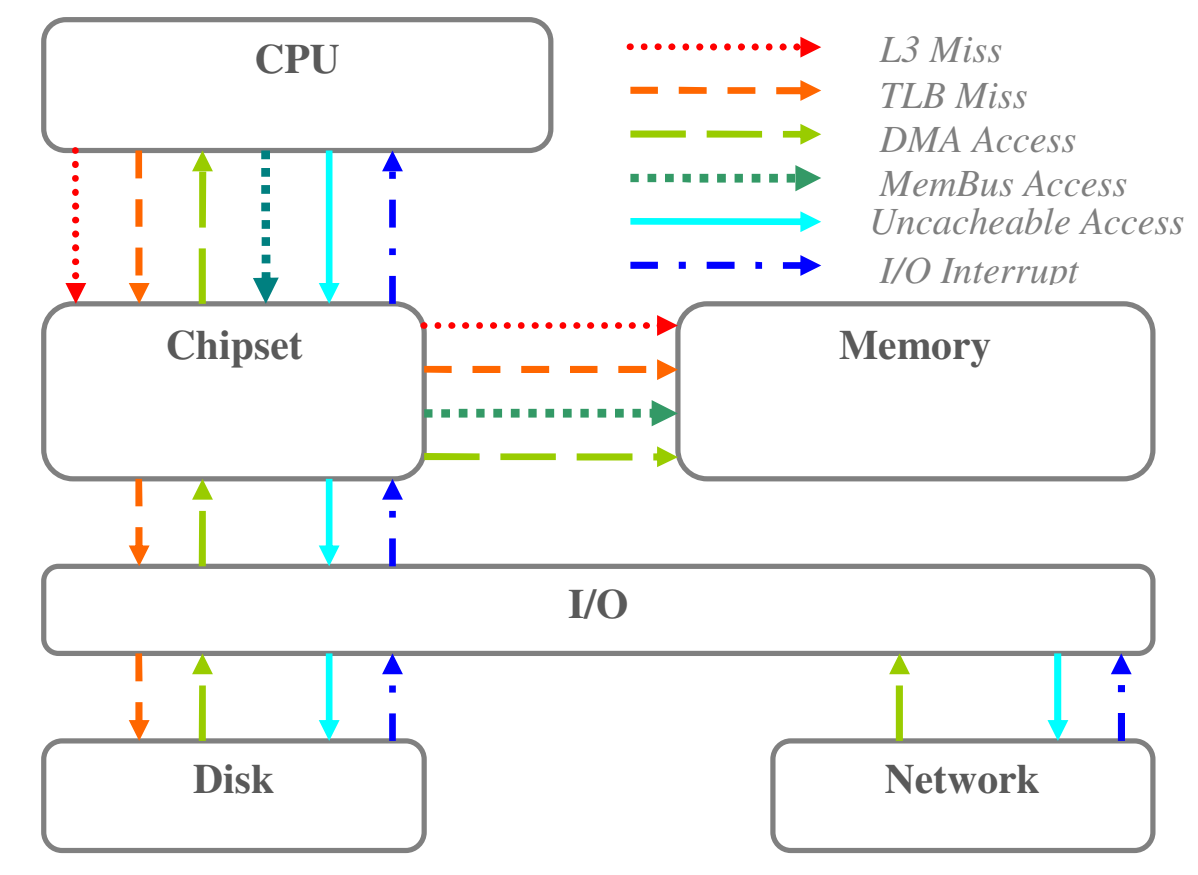

Figure 5.1. Propagation of Performance Events

With over forty detectable performance events [Sp02], the Pentium IV provides a challenge in selecting events that are most representative of subsystem power. The subsystem interconnections pictured in Figure 5.1 provide a starting point. By noting the "trickle-down" effect of events in the processor, a subset of the performance events is selected to accurately model subsystem power consumption. A simple example would be the effect of cache misses in the processor. For a typical microprocessor the top-level cache affects power consumption in the memory subsystem. Transactions that cannot be satisfied (cache miss) by the top-level cause a cache line (block) sized access to the main 
memory. Since the number of main memory accesses is directly proportional to the number of cache misses, it is possible to approximate the number of accesses using only cache misses. Since these memory accesses must go off-chip, power is consumed proportionally in the memory controller and DRAM. In reality the relation is not so simple, but there is still a strong causal relationship between cache misses and main memory accesses.

\subsection{Complete-System Server Power Model}

Though the initial selection of performance events for modeling is dictated by an understanding of subsystem interactions (as in the previous example), the final selection of which event type(s) to use is determined by the average error rate and a qualitative comparison of the measured and modeled power traces. The dominant, power-related performance events are described below.

Cycles - Execution time in terms of CPU clock cycles. The cycles metric is combined with most other metrics to create per cycle metrics. This corrects for slight differences in sampling rate. Though sampling is periodic, the actual sampling rate varies slightly due to cache effects and interrupt latency.

Halted Cycles - Cycles in which clock gating is active. When the Pentium IV processor is idle, it saves power by gating the clock signal to portions of itself. Idle phases of execution are "detected" by the processor through the use of the HLT (halt) instruction. 
When the operating system process scheduler has available slack time, it halts the processor with this instruction. The processor remains in the halted state until receiving an interrupt. Though the interrupt can be an I/O device, it is typically the periodic OS timer that is used for process scheduling/preemption. This has a significant effect on power consumption by reducing processor idle power from $\sim 36 \mathrm{~W}$ to $9 \mathrm{~W}$. Because this significant effect is not reflected in the typical performance metrics, it is accounted for explicitly in the halted cycles counter.

Fetched $\mu$ ops - Micro-operations fetched. The micro-operations ( $\mu$ ops) metric is used rather than an instruction metric to improve accuracy. Since in the P6 architecture instructions are composed of a varying number of $\mu$ ops, some instruction mixes give a skewed representation of the amount of computation being done. Using $\mu$ ops normalizes the metric to give representative counts independent of instruction mix. Also, by considering fetched rather than retired $\mu$ ops, the metric is more directly related to power consumption. Looking only at retired $\mu$ ops would neglect work done in execution of incorrect branch paths and pipeline flushes.

L3 Cache Misses - Loads/stores that missed in the Level 3 cache. Most system main memory accesses can be attributed to misses in the highest level cache, in this case L3. Cache misses can also be caused by DMA access to cacheable main memory by I/O devices. The miss occurs because the DMA must be checked for coherency in the processor cache. 
TLB Misses - Loads/stores that missed in the instruction or data Translation Lookaside Buffer. TLB misses are distinct from cache misses in that they typically cause trickledown events farther away from the microprocessor. Unlike cache misses, which usually cause a cache line to be transferred from/to memory, TLB misses often cause the transfer of a page of data (4KB or larger). Due to the large size of pages, they are often stored on disk. Therefore, power is consumed on the entire path from the CPU to the hard disk.

DMA Accesses - Transaction that originated in an I/O device whose destination is system main memory. Though DMA transactions do not originate in the processor, they are fortunately visible to the processor. As demonstrated in the L3 Miss metric description, these accesses to the processor (by an I/O device) are required to maintain memory coherency. Being able to observe DMA traffic is critical since it causes power consumption in the memory subsystem. An important thing to consider in the use of the Pentium IV's DMA counting feature is that it cannot distinguish between DMA and processor coherency traffic. All memory bus accesses that do not originate within a processor are combined into a single metric (DMA/Other). For the uniprocessor case this is not a problem. However, when using this metric in an SMP environment such as this, care must be taken to attribute accesses to the correct source. Fortunately, the workloads considered here have little processor-to-processor coherency traffic. This ambiguity is a limitation of the Pentium IV performance counters and is not specific to this technique.

Processor Memory Bus Transactions - Reads or writes on processor's external memory bus. All transactions that enter/exit the processor must pass through this bus. Intel calls 
this the Front Side Bus (FSB). As mentioned in the section on DMA, there is a limitation of being able to distinguish between externally generated (other processors) and DMA transactions.

Uncacheable Accesses - Load/Store to a range of memory defined as uncacheable. These transactions are typically representative of activity in the I/O subsystem. Since the I/O buses are not cached by the processor, downbound (processor to I/O) transactions and configuration transactions are uncacheable. Since all other address space is cacheable, it is possible to directly identify downbound transactions. Also, since configuration accesses typically precede large upbound (I/O to processor) transactions, it is possible to indirectly observe these.

Interrupts - Interrupts serviced by CPU. Like DMA transactions, most interrupts do not originate within the processor. In order to identify the source of interrupts, the interrupt controller sends a unique ID (interrupt vector number) to the processor. This is particularly valuable since I/O interrupts are typically generated by I/O devices to indicate the completion of large data transfers. Therefore, it is possible to attribute $\mathrm{I} / \mathrm{O}$ bus power to the appropriate device. Though, the interrupt vector information is available in the processor, it is not available as a performance event. Therefore, the presence of interrupt information in the processor simulated by obtaining it from the operating system. Since the operating system maintains the actual interrupt service routines, interrupt source accounting can be easily performed. In this case the /proc/interrupts file available in Linux operating systems is used. 
The form of the subsystem power models is dictated by two requirements: low computational cost and high accuracy. Since these power models are intended to be used for runtime power estimation, it is preferred that they have low computational overhead. For that reason regression curve fitting is attempted using single or multiple input linear models. If it is not possible to obtain high accuracy with a linear model, a single or multiple input quadratic is selected.

\section{Subsystem Power Models}

The following sections describe the details of the subsystem power models. Descriptions are given for issues encountered during the selection of appropriate input metrics. For each subsystem a comparison of modeled and measured power under a high variation workload is given.

\subsubsection{CPU}

This CPU power model improves an existing model [BiVa05] to account for halted clock cycles. Since it is possible to measure the percent of time spent in the halt state, it is possible to account for the greatly reduced power consumption due to clock gating. This addition is not a new contribution, since a similar accounting was made in the model by Isci [IsMa03]. The distinction is that this model is the first application of a performancebased power model in an SMP environment. The ability to attribute power consumption to a single physical processor within an SMP environment is critical for shared computing environments. In the near future it is expected that billing of compute time in 
these environments will take account of power consumed by each process [Mc04]. This is particularly challenging in virtual machine environments in which multiple customers could be simultaneously running applications on a single physical processor. For this reason, process-level power accounting is essential.

Given that the Pentium IV can fetch three instructions/cycle, the model predicts range of power consumption from 9.25 Watts to 48.6 Watts. The form of the model is given in Equation 5.1.

$$
\sum_{i=1}^{\text {NumCPUs }} 9.25+(35.7-9.25) \times \text { PercentActive }_{i}+4.31 \times \frac{\text { FetchedUops }_{i}}{\text { Cycle }}
$$

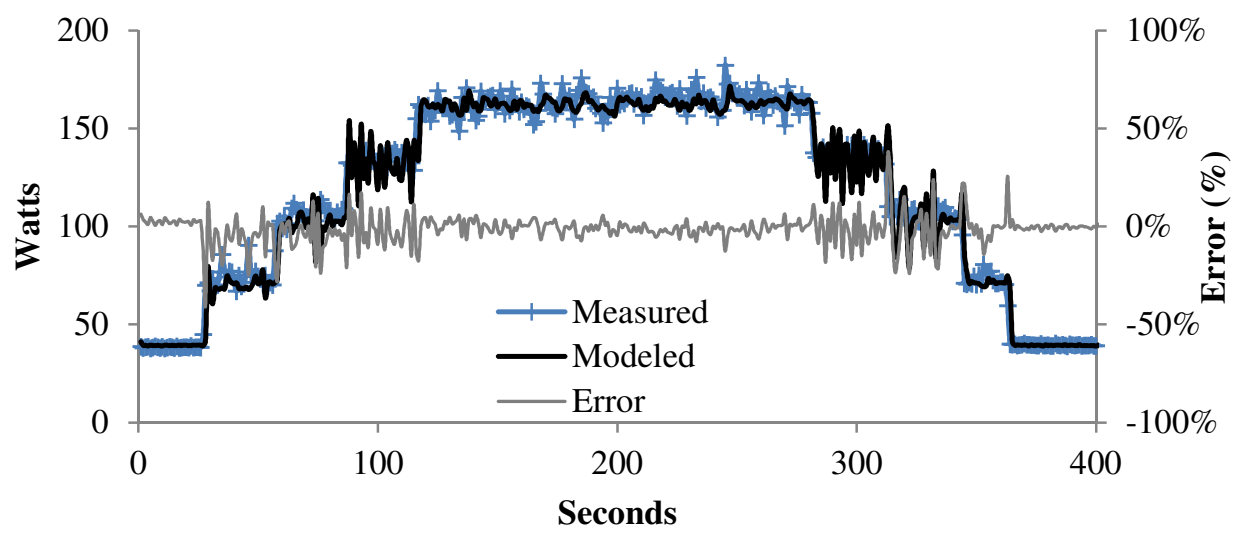

Figure 5.2 Processor Power Model - gcc

A trace of the total measured and modeled power for the four processors is given in Figure 5.2. The workload used in the trace is eight threads of gcc, started at 30s intervals. Average error is found to be $3.1 \%$. Note that unlike the memory bound workloads that saturate at eight threads, the cpu-bound gcc saturates after only 4 simultaneous threads. 


\subsubsection{Memory}

This section considers models for memory power consumption based on cache misses and processor bus transactions.

The initial attempt at modeling memory power made use of cache misses. A model based on only cache misses/cycle is an attractive prospect as it is a well understood metric and is readily available in performance monitoring counters. The principle behind using cache misses as proxy for power is that loads not serviced by the highest level cache, must be serviced by the memory subsystem. As demonstrated in [Ja01], power consumption in DRAM modules is highest when the module is in the active state. This occurs when either read or write transactions are serviced by the DRAM module. Therefore, the effect of high-power events such as DRAM read/writes can be estimated.

In this study, the number of L3 Cache load misses per cycle is used. Since the Pentium IV utilizes a write-back cache policy, write misses do not necessarily cause an immediate memory transaction. If the miss was due to a cold start, no memory transaction occurs. For conflict and capacity misses, the evicted cache block will cause a memory transaction as it updates memory.

The initial findings showed that L3 cache misses were strong predictors of memory power consumption (Figure 5.3). The first workload considered is the integer workload mesa from the SPEC CPU 2000 suite. Since a single instance of this workload does not sufficiently utilize the memory subsystem, multiple instances are used to increase 
utilization. For mesa, memory utilization increases noticeably with each instance of the workload. Utilization appears to taper off once the number of instances approaches the number of available hardware threads in the system. In this case the limit is 8 (4 physical processors x 2 threads/processor). The resultant quadratic power model is given in Equation 5.2. The average error under the mesa workload is low at only $1 \%$. However, the model fails under extreme cases.

$$
\sum_{i=1}^{\text {NumCPUs }} 28+\frac{\text { L3LoadMisses }_{i}}{\text { Cycle }} \times 3.43+\frac{\text { L3 }_{\text {LoadMisses }}{ }_{i}{ }^{2}}{\text { Cycle }} \times 7.66
$$

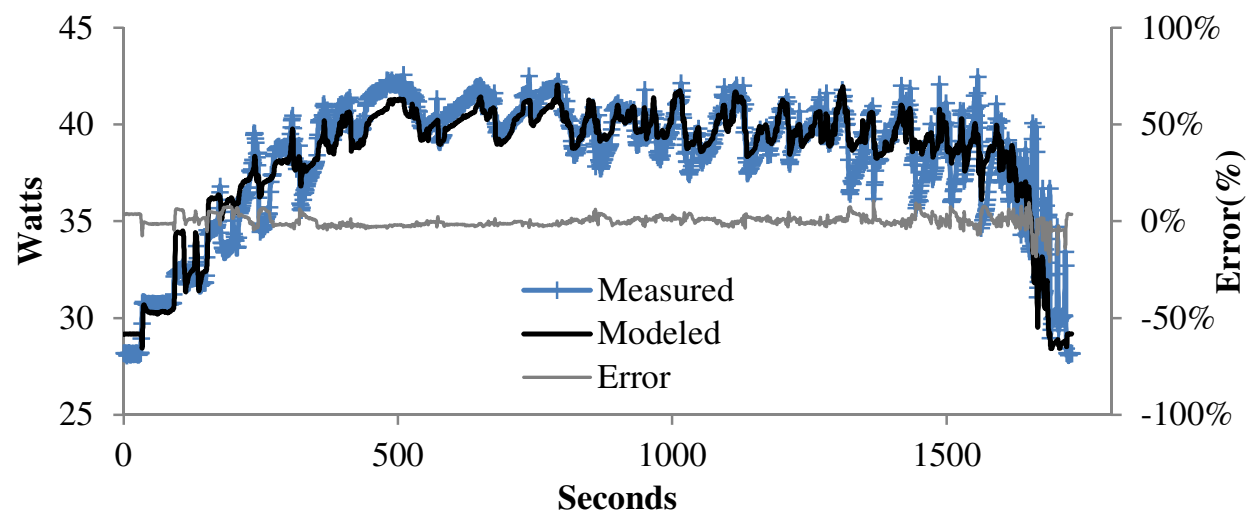

Figure 5.3 Memory Power Model (L3 Misses) - mesa

Unfortunately, L3 misses do not perform well under all workloads. In cases of extremely high memory utilization, L3 misses tend to underestimate power consumption. It is found that when using multiple instances of the mcf workload, memory power consumption continues to increase, while L3 misses are slightly decreasing.

It is determined that one of the possible causes is hardware-directed prefetches that are not accounted for in the count of cache misses. However, Figure 5.4 shows that though 
prefetch traffic does increase after the model failure, the total number of bus transactions does not. Since the number of bus transactions generated by each processor does not sufficiently predict memory power, an outside (non-CPU) agent is accessing the memory bus. For the target system the only other agent on the memory bus is the memory controller itself, performing DMA transactions on behalf of I/O devices.

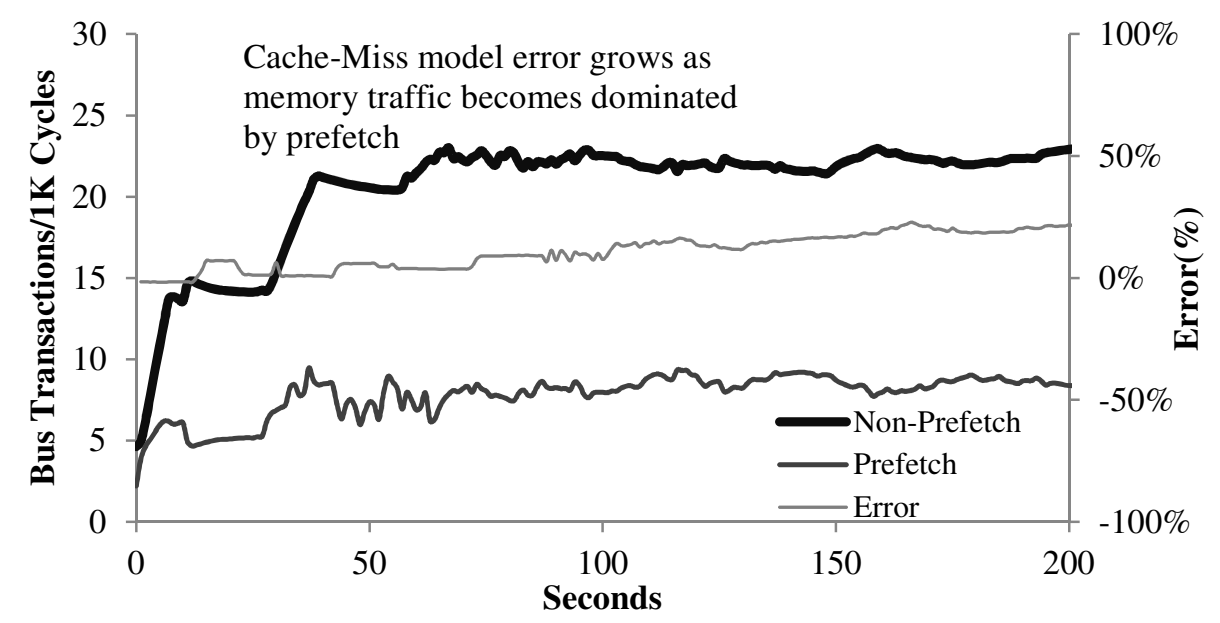

Figure 5.4 Prefetch and Non-Prefetch Bus Transactions - mcf

Changing the model to include memory accesses generated by the microprocessors and DMA events resulted in a model that remains valid for all observed bus utilization rates.

It should be noted that using only the number of read/write accesses to the DRAM does not directly account for power consumed when the DRAM is in the precharge state. DRAM in the precharge state consumes more power than in idle/disabled state, but less than in the active state. During the precharge state, data held in the sense amplifiers is committed to the DRAM array. Since the initiation of a precharge event is not directly controlled by read/write accesses, precharge power cannot be directly attributed to 
$\mathrm{read} / \mathrm{write}$ events. However, in practice read/write accesses are reasonable predictors. Over the long term (thousands of accesses) the number of precharge events should be related to the number of access events. The resultant model is given in Equation 5.3. A trace of the model applied to the mcf workload is shown in Figure 5.5. This workload cannot be modeled using cache misses. The model yields an average error rate of $2.2 \%$.

$$
\sum_{i=1}^{\text {NumCPUs }} 29.2-\frac{\text { BusTransađions }_{i}}{\text { MCycle }} \times 50.1 \cdot 10^{-4}+\frac{\text { BusTransađions }_{i}{ }^{2}}{\text { MCycle }} \times 813 \cdot 10^{-8}
$$

\subsubsection{Disk}

The modeling of disk power at the level of the microprocessor presents two major challenges: large distance from CPU to disk and little variation in disk power consumption. Of all the subsystems considered in this study, the disk subsystem is at the greatest time and distance from the microprocessor.

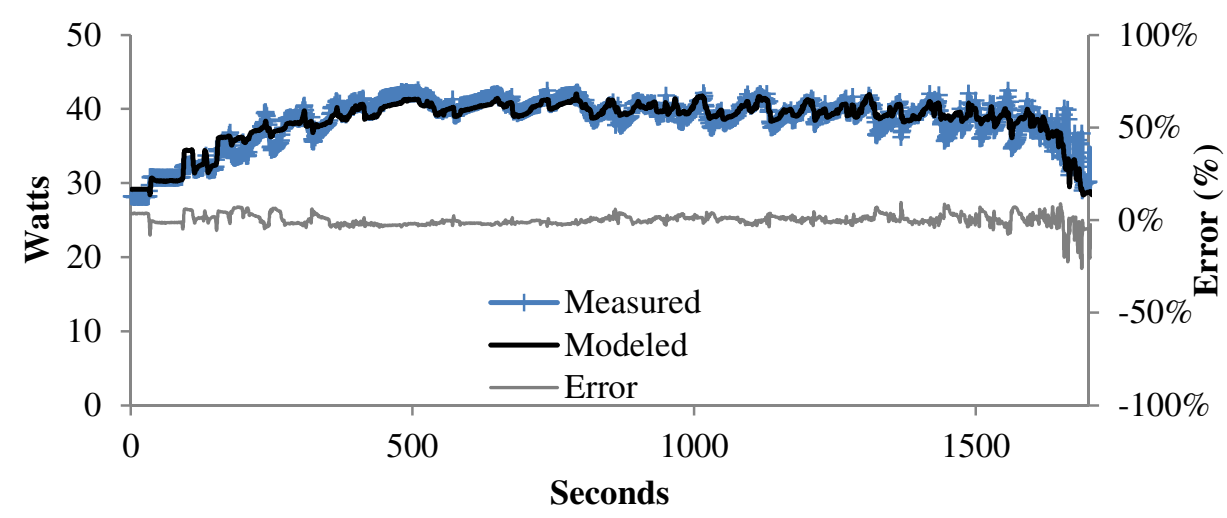

Figure 5.5 Memory Power Model (Memory Bus Transactions)- mcf

Therefore, there are challenges in getting timely information from the processor's perspective. The various hardware and software structures that are intended to reduce the 
average access time to the distant disk by the processor make power modeling difficult. The primary structures are: microprocessor cache, operating system disk cache, I/O queues and I/O and disk caches. The structures offer the benefit of decoupling highspeed processor events from the low-speed disk events. Since the power modeling techniques rely on the close relationship between the subsystems, this is a problem.

This is evidenced in the poor performance of the first attempts. Initially, we considered two events: DMA accesses and uncacheable accesses. Since the majority of disk transfers are handled through DMA by the disk controller, this appeared to be a strong predictor. The number of uncacheable accesses by the processor was also considered. Unlike the majority of application memory, memory mapped I/O (I/O address mapped to system address space) is not typically cached. Generally, I/O devices use memory mapped I/O for configuration and handshaking. Therefore, it should be possible to detect accesses to the I/O devices through uncacheable accesses. In practice neither of these metrics fully account for fine-grain power behavior. Since such little variation exists in the disk power consumption it is critical to accurately capture the variation that does exist. In this case the lack of variation is due to a lack of power management features. The addition of power management magnifies the variation present in the workload. Accounting for and modeling the small variation in the absence of power management suggests an accurate model can be constructed with power management.

To address this limitation the manner in which DMA transactions are performed is noted. Coarsely stated, DMA transactions are initiated by the processor by first configuring the 
I/O device. The transfer size, source and destination are specified through the memory mapped I/O space. The disk controller performs the transfer without further intervention from the microprocessor. Upon completion or incremental completion (buffer full/empty) the I/O device interrupts the microprocessor. The microprocessor is then able to use the requested data or discard local copies of data that was sent. This study uses the number of interrupts originating from the disk controller. This approach has the advantage over the other metrics in that the events are specific to the subsystem of interest. This approach is able to represent fine-grain variation with low error. In the case of the synthetic disk workload, by using the number of disk interrupts/cycle an average error rate of $1.75 \%$ is achieved. The model is provided in Equation 5.4. An application of the model to the memory-intensive mcf is shown in Figure 5.6. Note that this error rate accounts for the large DC offset within the disk power consumption. This error is calculated by first subtracting the $21.6 \mathrm{~W}$ of idle (DC) disk power consumption. The remaining quantity is used for the error calculation.

$$
\begin{aligned}
& \sum_{i=1}^{\text {NumCPUs }} 21.6+\frac{\text { Interrupts }_{i}}{\text { Cycle }} \times 10.6 \cdot 10^{7}-\frac{\text { Interrupt }_{i}{ }^{2}}{\text { Cycle }} \times 11.1 \cdot 10^{15} \\
& +\frac{\text { DMAAccess }_{i}}{\text { Cycle }_{i}} \times 9.18-\frac{\text { DMAAccess }_{i}{ }^{2}}{\text { Cycle }} \times 45.4
\end{aligned}
$$




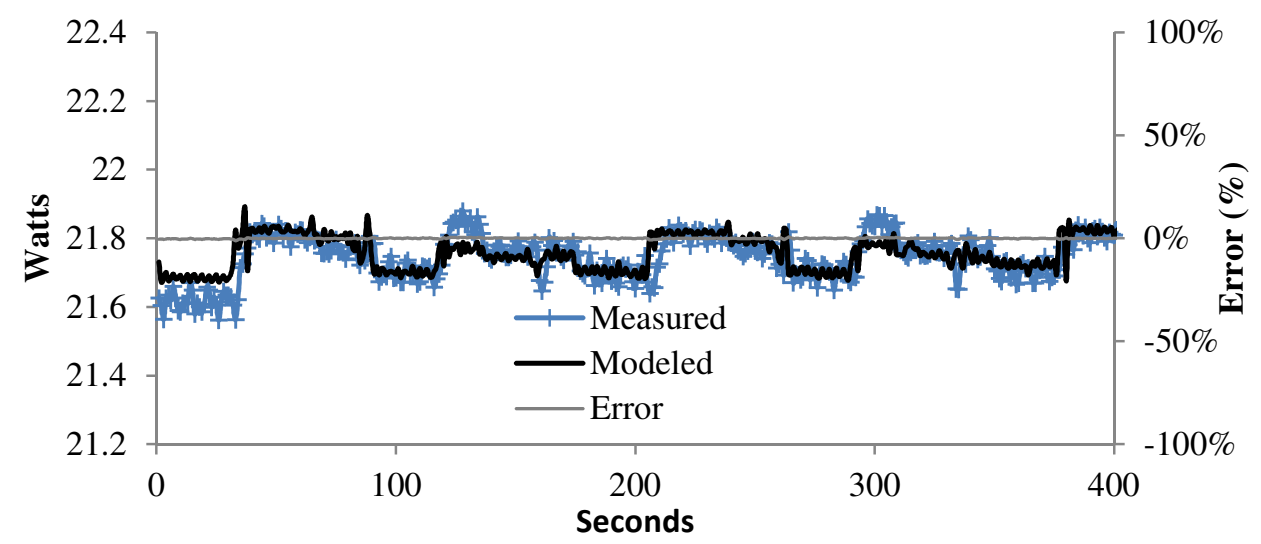

Figure 5.6 Disk Power Model (DMA+Interrupt) - Synthetic Disk Workload

\section{$5.2 .4 \mathrm{I} / \mathrm{O}$}

Since the majority of I/O transactions are DMA transactions from the various I/O controllers, an I/O power model must be sensitive to these events. Three events are considered to observe DMA traffic: DMA accesses on memory bus, uncacheable accesses and interrupts. Of the three, interrupts/cycle is the most representative. DMA accesses to main memory seemed to be the logical best choice since there is such a close relation to the number of DMA accesses and the switching factor in the I/O chips. For example, a transfer of cache line aligned 16 dwords ( 4 bytes/dword), maps to a single cache line transfer on the processor memory bus. However, in the case of smaller, nonaligned transfers the linear relationship does not hold. A cache line access measured as a single DMA event from the microprocessor perspective may contain only a single byte. This would grossly overestimate the power being consumed in the I/O subsystem. Further complicating the situation is the presence of performance enhancements in the I/O chips. 
One of the common enhancements is the use of write-combing memory. In writecombining, the processor or I/O chip in this case combines several adjacent memory transactions into a single transaction further removing the one-to-one mapping of $\mathrm{I} / \mathrm{O}$ traffic to DMA accesses on the processor memory bus. As a result, interrupt events are better predictors of I/O power consumption. DMA events failed to capture the fine-grain power variations. DMA events tended to have few rapid changes, almost as if the DMA events had a low-pass filter applied to them. The details of the model can be seen in Equation 5.5. Accounting for the large DC offset increases error significantly to $32 \%$. Another consideration with the model is the $\mathrm{I} / \mathrm{O}$ configuration used. The model has a significant idle power which is related to the presence to two I/O chips capable of providing six $133 \mathrm{MHz}$ PCI-X buses. While typical in servers, this is not common for smaller scale desktop/mobile systems that usually contain 2-3 I/O buses and a single I/O chip. Further, the server only utilizes a small number of the $\mathrm{I} / \mathrm{O}$ buses present. It is expected that with a heavily populated, system with fewer I/O buses, the DC term would become less prominent. This assumes a reasonable amount of power management within the installed I/O devices.

$$
\sum_{i=1}^{\text {NumCPUs }} 32.7+\frac{\text { Interrupt }_{i}}{\text { Cycle }} \times 108 \cdot 10^{6}-\frac{\text { Interrupt }_{i}{ }^{2}}{\text { Cycle }} \times 1.12 \cdot 10^{9}
$$

\subsubsection{Chipset}

The chipset power model is the simplest of all subsystems since a constant is all that is required for accuracy. There are two reasons for this. First, the chipset subsystem 
exhibits little variation in power consumption. Therefore, a constant power model is an obvious choice. Further, it is difficult to identify the effect performance events have on power consumption compared to induced electrical noise in the sensors. The second, and more critical reason, is a limitation in the power sampling environment. Since the chipset subsystem uses power from more than one power domain, the total power cannot be measured directly. Instead, it is derived by finding the average measured difference in power between multiple domains. The average chipset power is $19.9 \mathrm{~W}$.

\subsubsection{Model Validation}

Tables 5.1 and 5.2 present summaries of average errors for the five models applied to twelve workloads. Errors are determined by comparing modeled and measured error at each sample. A sample corresponds to one second of program execution or approximately 1.5 billion instructions per processor. For performance counter sampling, the total number of events during the previous one second is used. For power consumption, the average of all samples in the previous second (ten thousand) is used. One second sample intervals provide a compromise between trace size and accuracy. Reducing the sample interval to as low as 100 microseconds does increase the magnitude of error in worst-case samples. However, the cumulative average error as shown in Equation 5.6 is nearly identical to that obtained with one second sample intervals. The benefit is that trace size is reduced to a practical level that allows tracing complete, realistic workloads. 


$$
\text { AverageEror }=\frac{\sum_{i=1}^{\text {NumSamples } \mid} \frac{\mid \text { Modeled }_{i}-\text { Measured }_{i} \mid}{\text { Measured }_{i}}}{\text { NumSamples }} \times 100 \%
$$

The I/O and disk models performed well under all workloads. The low error rates are partly due to low power variation / high idle power consumption. The CPU and memory subsystems had larger errors, but also larger workload variation. The worst case errors for CPU occurred in the memory-bound workload: mcf. Due to a high CPI (cycles/instruction) of greater than ten cycles, the fetch-based power model consistently underestimates CPU power. This is because under mcf the processor only fetches one instruction every 10 cycles even though it is continuously searching for (and not finding) ready instructions in the instruction window. For mcf this speculative behavior has a high power cost that is equivalent to executing an additional 1-2 instructions/cycle.

Table 5.1 Integer Average Model Error

\begin{tabular}{|c|c|c|c|c|c|}
\hline Workload & CPU & Chipset & Memory & I/O & Disk \\
\hline Idle & $1.74 \%$ & $0.586 \%$ & $3.80 \%$ & $0.356 \%$ & $0.172 \%$ \\
\hline Gcc & $4.23 \%$ & $10.9 \%$ & $10.7 \%$ & $0.411 \%$ & $0.201 \%$ \\
\hline Mcf & $12.3 \%$ & $7.7 \%$ & $2.2 \%$ & $0.332 \%$ & $0.154 \%$ \\
\hline Vortex & $6.53 \%$ & $13.0 \%$ & $15.6 \%$ & $0.295 \%$ & $0.332 \%$ \\
\hline DBT-2 & $9.67 \%$ & $0.561 \%$ & $2.17 \%$ & $5.62 \%$ & $0.176 \%$ \\
\hline SPECjbb & $9.00 \%$ & $7.45 \%$ & $6.14 \%$ & $0.393 \%$ & $0.144 \%$ \\
\hline DiskLoad & $5.93 \%$ & $3.06 \%$ & $2.93 \%$ & $0.706 \%$ & $0.161 \%$ \\
\hline Integer Average & 7.06 & $6.18 \%$ & $6.22 \%$ & $1.16 \%$ & $0.191 \%$ \\
& $\pm 3.50 \%$ & $\pm 4.92 \%$ & $\pm 5.12 \%$ & $\pm 1.97 \%$ & $\pm 0.065 \%$ \\
\hline \multirow{2}{*}{ All Workload Average } & $6.67 \%$ & $5.97 \%$ & $8.80 \%$ & $0.824 \%$ & $0.390 \%$ \\
& $\pm 3.42 \%$ & $\pm 4.23 \%$ & $\pm 5.54 \%$ & $\pm 1.52 \%$ & $\pm 0.492 \%$ \\
\hline
\end{tabular}


The memory model averaged about $9 \%$ error across all workloads. Surprisingly the memory model fared better under integer workloads. The error rate for floating point workloads tended to be highest for workloads with the highest sustained power consumption. For these cases the model tends to underestimate power. Since the rate of bus transactions is similar for high and low error rate workloads it is suspected that the cause of underestimation is the access pattern. In particular the model does not account for differences in the power for read versus write access. Also, the number of active banks within the DRAM is not directly accounted for. Accounting for the mix of reads versus writes would be a simple addition to the model. However, accounting for active banks will likely require some form of locality metric.

Idle power error is low for all cases indicating a good match for the DC term in the models. Chipset error is high considering the small amount of variation. This is due to the limitation of the constant model assumed for chipset power.

Table 5.2 Floating Point Average Model Error

\begin{tabular}{|c|c|c|c|c|c|}
\hline Workload & CPU & Chipset & Memory & I/O & Disk \\
\hline Art & $9.65 \%$ & $5.87 \%$ & $8.92 \%$ & $0.240 \%$ & $1.90 \%$ \\
\hline Lucas & $7.69 \%$ & $1.46 \%$ & $17.5 \%$ & $0.245 \%$ & $0.31 \%$ \\
\hline Mesa & $5.59 \%$ & $11.3 \%$ & $8.31 \%$ & $0.334 \%$ & $0.17 \%$ \\
\hline mgrid & $0.360 \%$ & $4.51 \%$ & $11.4 \%$ & $0.365 \%$ & $0.55 \%$ \\
\hline wupwise & $7.34 \%$ & $5.21 \%$ & $15.9 \%$ & $0.588 \%$ & $0.42 \%$ \\
\hline FP Average & $6.13 \%$ & $5.67 \%$ & $12.41 \%$ & $0.354 \%$ & $0.67 \%$ \\
\hline All Workload & $\pm 3.53 \%$ & $\pm 3.57 \%$ & $\pm 4.13 \%$ & $\pm 0.142 \%$ & $\pm 0.70 \%$ \\
\hline Average & $6.67 \%$ & $5.97 \%$ & $8.80 \%$ & $0.824 \%$ & $0.39 \%$ \\
\hline & $\pm 3.42 \%$ & $\pm 4.23 \%$ & $\pm 5.54 \%$ & $\pm 1.52 \%$ & $\pm 0.49 \%$ \\
\hline
\end{tabular}




\subsection{Complete-System Desktop Power Model}

In this section results for the application of the trickle-down modeling approach are presented for a recent desktop platform. This platform differs from the previous server in terms of process technology, system architecture, manufacturer and workload among others. It is shown that though this platform is significantly different than the server, the trickle-down modeling approach still accurately models power. Of particular importance are two major differences: subsystem level power management and workload characteristics. Power management increases the complexity and utility of the power model as power consumption varies greatly with the application of power management. Compare this to the server system in which power remains near a constant level due to subsystems not reducing performance capacity, and therefore power consumption, during periods of low utilization. Increased power variation is also attributable to desktopspecific workloads. While server workloads tend to always operate at full speed (e.g. SPEC CPU) desktop workloads such as SYSmark and 3DMark contain large portions of low utilization. This exposes the impact of power management and the need to model it.

\subsubsection{System Description}

To validate the effectiveness of the trickle-down approach the process is applied to a recent desktop platform. A comparison of the two systems used in this study (server and desktop) is provided in Table 5.3. These systems differ in their power management implementations and subsystem components. The desktop system is optimized for power 
efficiency rather than performance. This leads to greater variation in power consumption compared to a server since power management features reduce power greatly during low utilization. Server systems tend to employ less aggressive power savings. Therefore, power at low utilization is greater and overall variation is lesser. This difference is evident in the analysis of average subsystem-level power in Tables 5.1 - 5.2 and 5.6. The power management implementation in the desktop system also requires the use of more extensive power models. Rather than only needing to consider CPU clock gating and DRAM power down modes, the desktop system model must consider DVFS, chipset link power management, disk and GPU power management. The wider range of power consumption also leads to greater temperature sensitivity.

Table 5.3 System Comparison

\begin{tabular}{|c|c|c|}
\hline Platform & Server & Desktop \\
\hline Segment & Intel & AMD \\
\hline Manufacturer & Quad-socket 130nM & Dual-core 45nM 2.0GHz \\
\hline Processor(s) & $2.8 \mathrm{GHz}$ & 4GB DDR3-1066 \\
\hline Memory & 8GB DDR-200 & CPU Clock Gating and DVFS \\
\hline Power & CPU Clock Gating & Refresh \\
Management & DRAM Power Down & DRAM Power Down and Self \\
& & Chipset Link Disconnect \\
& & Harddrive Spin Down and \\
& & ATA modes \\
\hline Graphics & & GPU Clock Gating \\
\hline Observable & Rage ProXL & RS780 \\
Subsystems & CPU & CPU \\
& Chipset & Chipset \\
& Memory & Memory \\
& I/O & Memory Controller \\
& Disk & GPU \\
\hline & & Disk \\
\hline
\end{tabular}


Another major difference is the ability to measure subsystem power at a finer granularity. The desktop platform allows direct measurement of memory controller and GPU in addition to all the subsystems that are measureable in the server system. One exception is the server I/O subsystem which contains numerous PCI-X busses and bridges. The desktop system does not contain comparable I/O subsystem. Therefore, it is not included in the study.

\subsubsection{Workloads}

Due to the distinctions between server and desktop systems several desktop or clientappropriate workloads are added. In typical server or desktop benchmarks the GPU subsystem is almost entirely idle. Therefore, to exercise the GPU subsystem the 3DMark06 benchmark is included. 3DMark06 contains six subtests covering CPU and GPU intensive workloads. Four of the subtests target the GPU's 3D processing engine (gt1, gt2, hdr1, hdr2). The other two (cpu1 and cpu2) heavily utilize CPU cores but have almost no GPU utilization. Targeting the 3D engine generates the largest power variation since the 3D engine is by far the largest power consumer in the GPU. An interesting side effect of the desktop GPU is intense system DRAM utilization. To reduce cost and power consumption, desktop systems such as this use a portion of system DRAM in lieu of locally attached, private DRAM. As a result, 3D workloads in desktop systems are effective at generating wide power variation in the memory subsystem.

Overall subsystem level power management is exposed through the addition of the SYSmark 2007 benchmark. This workload is implemented using simulated user input 
through the application GUI. The numerous delays required for GUI interaction causes many idle phases across the subsystems. This allows power management to become active. Contrast this to the vast majority of benchmarks which, by design, operate the CPU and other subsystems only at the $100 \%$ load level. The DBT-2 database workload is excluded as it is not practical and relevant to run on a desktop platform. For comparison to the server model, SPEC CPU, SPECjbb and Idle workloads are included. The workloads on targeted subsystems are summarized below in Table 5.4.

\section{Table 5.4 Desktop Workloads}

\begin{tabular}{|c|c|c|c|}
\hline \multicolumn{2}{|l|}{ Workload } & Description & Subsystems Targeted \\
\hline \multicolumn{2}{|l|}{ Idle } & Only background OS processes & All (power managed) \\
\hline \multirow{2}{*}{ SPEC CPU 2006} & INT & $\begin{array}{l}\text { perlbench, bzip2, gcc, mcf, } \\
\text { gobmk, hmmer, sjeng, libquantum, } \\
\text { h264ref, omnetpp, astar, } \\
\text { xalancbmk }\end{array}$ & $\begin{array}{c}\text { CPU } \\
\text { Memory } \\
\text { Memory Controller }\end{array}$ \\
\hline & FP & $\begin{array}{l}\text { bwaves, games, milc, zeusmp, } \\
\text { gromacs, cactusADM, leslie3d, } \\
\text { namd, dealII, soplex, povray, } \\
\text { calculix, gemsFDTD, tonto, lbm, } \\
\text { wrf, sphinx3 }\end{array}$ & $\begin{array}{c}\text { CPU } \\
\text { Memory } \\
\text { Memory Controller }\end{array}$ \\
\hline \multirow{5}{*}{ 3DMark06 } & gt1 & \multirow[b]{2}{*}{ Graphics Test 1 and 2} & \multirow{2}{*}{$\begin{array}{c}\text { GPU } \\
\text { Memory } \\
\text { Memory Controller }\end{array}$} \\
\hline & $\mathrm{gt} 2$ & & \\
\hline & $\begin{array}{l}\text { cpu1 } \\
\text { cpu2 }\end{array}$ & CPU Test 1 and 2 & $\mathrm{CPU}$ \\
\hline & hdr1 & \multirow{2}{*}{ High Dynamic Range Test 1 and 2} & \multirow{2}{*}{$\begin{array}{c}\text { GPU } \\
\text { Memory } \\
\text { Memory Controller }\end{array}$} \\
\hline & hdr2 & & \\
\hline \multirow{4}{*}{ SYSmark 2007} & EL & E-Learning & \multirow{4}{*}{$\begin{array}{c}\text { CPU } \\
\text { Memory } \\
\text { Memory Controller } \\
\text { Chipset } \\
\text { Disk }\end{array}$} \\
\hline & $\mathrm{VC}$ & Video Creation & \\
\hline & PR & Productivity & \\
\hline & $3 \mathrm{D}$ & $3 \mathrm{D}$ & \\
\hline \multicolumn{2}{|c|}{ SPECjbb2005 } & Server-Side Java & $\begin{array}{c}\text { CPU } \\
\text { Memory } \\
\text { Memory Controller }\end{array}$ \\
\hline
\end{tabular}




\subsubsection{Performance Event Selection}

Performance event selection is critical to the success of performance counter-driven power models. To identify a minimum set of representative performance events, the relationship between each event and power consumption must be understood. This section describes the performance monitoring counter events used to construct the trickledown power model. The definition and insight behind selection of the counters is provided.

Fetched $\mu$ ops - Micro-operations fetched. Comparable to the Pentium IV fetched microoperations, this metric is highly correlated to processor power. It accounts for the largest portion of core pipeline activity including speculation. This is largely the result of finegrain clock gating. Clocks are gated to small portions of the pipelines when they are not being used.

FP $\mu$ ops Retired - Floating point micro-operations retired. FP $\mu$ ops Retired accounts for the difference in power consumption between floating point and integer instructions. Assuming equal throughput, floating point instructions have significantly higher average power. Ideally, the number of fetched FPU $\mu$ ops would be used. Unfortunately, this metric is not available as a performance counter. This is not a major problem though since the fetched $\mu$ ops metric contains all fetched $\mu$ ops, integer and floating point.

DC Accesses - Level 1 Data Cache Accesses. A proxy for overall cache accesses including Level 1,2,3 data and instruction. Considering the majority of workloads, level 
1 data cache access rate dominates cache-dependent power consumption. No other single cache access metric correlates as well to processor core power (including caches).

$\%$ Halted/\% Not-Halted - Percent time processor is in halted state. This represents power saved due to explicit clock gating. The processor saves power using fine-grain and coarse-grain of clock gating. Fine-grain clock gating saves power in unutilized portions of the processor while instructions are in-flight. Coarse-grain clock gating can save more power than fine-grain yet it requires the processor to be completely idle. The processor applies this type of gating only when the processor is guaranteed to not have any instructions in-flight. This condition by definition occurs following execution of the HLT instruction. Halt residency is controlled by the operating system and interrupts scheduling work on processors.

CPU Clock Frequency - Core clocks per second. Due to the use of DVFS, it is necessary to track the instantaneous frequency of the processor. Though some metrics such as $\mu$ ops fetched or retired implicitly track the power consumed in many components due to clock frequency, they do not track workload-independent power consumers such as clock grids. Using clock frequency in conjunction with \%Halt it is possible to account for power consumed in these units.

CPU Voltage - CPU Voltage Rail. Due to the application of DVFS the processor may operate at a range of discrete voltages in order to save power. Changes in voltage have a significant impact on power consumption due to the exponential relationship between 
voltage and dynamic $\left(\sim V^{2}\right)$ and leakage power $\left(\sim V^{3}\right)$. Due to a single, shared voltage plane, the actual voltage applied is the maximum requested of all cores in a socket. The requested voltage can be read using the P-State Status Register [Bk09].

Temperature - CPU Temperature. At the high voltages required for multi-GHz operation, leakage power becomes a major component of power consumption. Also, at idle when dynamic power is nearly eliminated due to clock gating leakage power can be the dominant contributor. Since temperature has a strong relation to leakage power $\left(\mathrm{T}^{2}\right)$ it is necessary to account for this effect by measuring temperature. Temperature can be approximated using a series of on-die thermal sensors. The output of these sensors can be obtained using a configuration-space register [Bk09].

GPU Non-Gated Clocks - Number of GPU clocks per second. Similar to CPU power, GPU power is greatly impacted by the amount of clock gating and DVFS. In this study the DVFS usage is restricted to frequency changes only. Therefore, nearly all GPU power variation can be accounted for by this single metric.

DCT Accesses $-\sum_{N=0-1} D C T_{N}$ PageHits $+D C T_{N}$ PageMisses $+D C T_{N}$ PageConflicts. DCT (DRAM ConTroller) Access accounts for all memory traffic flowing out of the two ondie memory controllers, destined for system DRAM. These events include cpu-generated and DMA traffic.

Link Active\% - Percent time Hypertransport links connected. To save power in the I/O interconnection during idle periods, the Hypertransport links are disconnected. During 
periods of disconnect, cache snoop traffic and interrupts are blocked. This allows power to be saved in the CPU I/O interconnect and I/O subsystem. Also, the DRAM may be placed in self-refresh mode since DRAM access is blocked. If a cache snoop or interrupt event occurs, the links are reconnected.

Spindle Active \% - Percent time hard disk spindle is spinning. In traditional mechanical hard drives, the spindle motor represent the largest single consumer of power in the drive. To save energy the spindle motor can be powered down. Due to the high latency (and energy consumption) for starting/stopping the spindle this can only be done when the drive is expected to be idle for a long time (minutes or more). In practice, typical workloads prevent the spindle from ever powering down. This includes all benchmarks used in this study, except idle. Therefore, spindle activity can be sufficiently accounted for by only distinguishing between idle and active workloads.

CPUToIOTransactions - Non-cacheable access to memory-mapped I/O devices . I/O device activity can be approximated using a measure of how many memory transactions generated by the CPUs are targeted at non-cacheable address space. Typically, I/O devices contain a DMA controllers which performs access to cacheable space in system memory. The configuration and control of these transactions is performed by the CPU through small blocks of addresses mapped in non-cacheable space to each I/O device.

DRAMActive\% - Percent time DRAM channel is active. Power savings in the DRAM and memory controller is controlled by the memory controller. When a memory channel 
has not issued a memory transaction for at least fixed period of time, the memory controller sends the channel to one of the precharge power down modes [Bk09]. This primarily saves power in the DRAM chips, but also provides a slight savings in the memory controller.

\section{SUBSYSTEM POWER MODELS}

The impact of effective power management can be seen in the form of the power models of this section. In all cases it is necessary to explicitly account for power management to obtain accurate models. This causes all models to take a similar form. Previous models [BiVa05] [BiJo06-1] are dominated by terms which were directly proportional to workload activity factors (IPC, cache accesses). While those workload-dependent terms are also used here, Idle Power Management and Irreducible power are also quantified. The idle power management term estimates power saved when instructions or operations are not actively proceeding through the subsystem. For CPUs this primarily occurs when executing the idle loop. The CPU detects one of the idle instructions (HLT, mwait) and takes actions such as clock or power gating. Other subsystems such as memory or $\mathrm{I} / \mathrm{O}$ links similarly detect the absence of transactions and save power through various degrees of clock gating. Irreducible power contains the "baseline" power which is consumed at all times. This baseline power is largely composed of leakage and ungateable components. 


\subsubsection{CPU}

To test the extensibility of performance counter power modeling across processor architectures, the methodology is applied to an AMD Phenom quad-core processor. Like the Intel Pentium 4 processor used in sections 3.2 and 3.3, fetched instructions is a dominant metric for power accounting. Differences in architecture and microarchitecture dictate that additional metrics are needed to attain high accuracy. Two areas are prominent: floating point instruction power and architectural power management. Unlike the Intel server processor which exhibits nearly statistically uniform power consumption across workloads of similar fetch rate, the AMD desktop processor consumes up to $30 \%$ more power for workloads with large proportions of floating point instructions.

To account for the difference in floating point instruction power, the desktop processor model employs an additional metric for retired floating point instructions. A still larger power difference is caused by the addition of architectural power management on the desktop processor. The older, server processor only has architectural power management in the form of clock gating when the halt instruction is issued by the operating system. The newer, desktop processor adds architectural, DVFS. This leads to drastic reductions in switching and leakage power. To account for these power reductions, the desktop model includes tracking of processor frequency, voltage and temperature. The details of the model can be found in Section 3.4.5. 
While this model is accurate for most current generation processors, future processors may require additional metrics to maintain comparable accuracy. Power savings techniques such as power gating and on-die voltage regulation will require new methods for power accounting. These techniques extend the sensitivity of leakage power consumption to functional unit activity levels. Currently, leakage power is dictated almost completely by architecturally visible, core-level, idle and DVFS states. Future power gating implementations will likely be applied within subsets of a core, such as floating point units or caches. Similarly, on-die regulation allows DVFS to be applied independently to particular functional units. This increases the complexity of performance counter power modeling which normally only accounts for switching power. To accounting for these local power adaptations, models will need either detailed knowledge of the power gating and DVFS implementations or statistical characterizations of their application. Given the effectiveness of performance counter power models at accounting for fine-grain switching power, it is likely that power gating and on-die regulation can also be accounted for.

\subsubsection{GPU}

To estimate GPU power consumption a technique similar to that typically used for CPUs is employed: count the number of ungated clocks. In CPUs this is done by subtracting the number of halted clocks from all clocks [BiJo06-1]. In the case of the RS780 the ungated clocks can be measured directly. This approach only accounts directly for power saved due to clock gating. Power reductions due to DVFS are not explicitly represented. 
Despite this, high accuracy of less than $1.7 \%$ error is obtained due to the implementation of DVFS. Unlike CPU DVFS which allows the operating system to reduce voltage and frequency during active phases, GPU DVFS reduces voltage only when clock gating is applied (idle). Therefore, increased power due to operating at the higher voltage is included in the non-gated clock metric. This bi-modal behavior can be seen in Figure 5.7. The mostly-idle, clock-gated portion of the HDR1 workload draws about 1.5W. The fully active phase increases voltage and eliminates clock gating. Power increases drastically to over $4 \mathrm{~W}$.

An alternative metric for GPU power was also considered: \% GUI Active. This metric represents the portion of time in which the GPU is updated the display. The main limitation of this approach is that it does not account for the intensity of work being performed by the underlying GPU hardware. Low-power 2D workloads, such as low-bit rate video playback, appear to have the same GPU utilization as more intense highresolution video decoding. An example of modeled versus measured GPU power for 3DMark06-HDR1 is provided in Figure 5.7. Modeled GPU power as a function of nongated GPU clocks is shown by Equation 5.7.

\section{GPU Power $=$}

$0.0068 \times($ Non-Gated Clocks $/ \mathrm{sec}) \times 10^{-6}+0.8467$ 


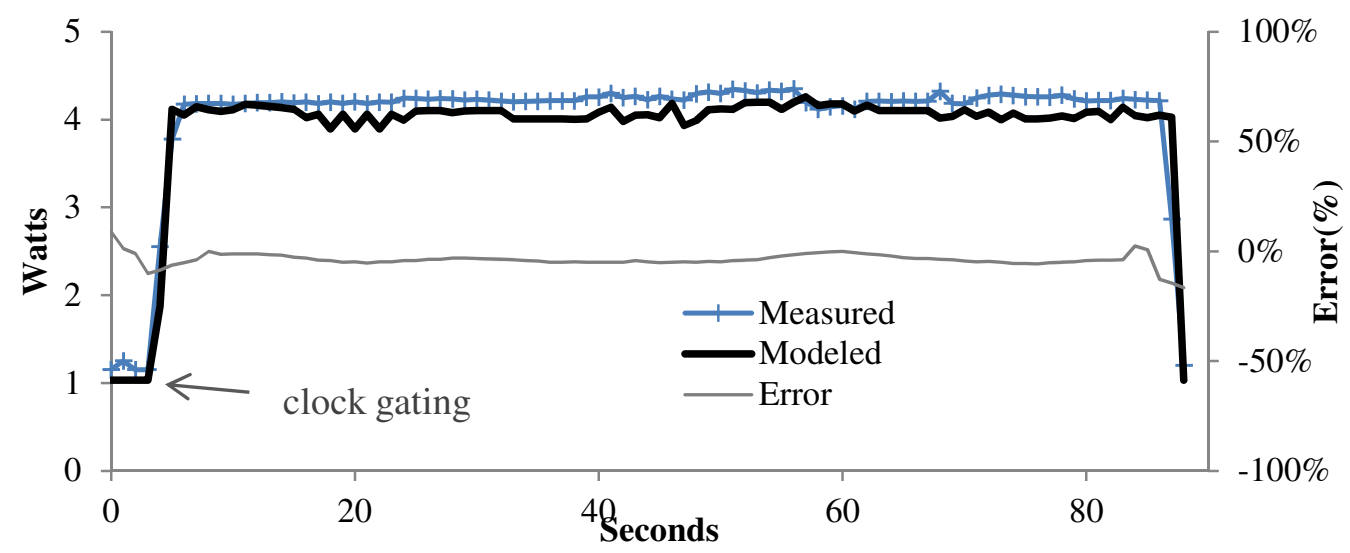

Figure 5.7 GPU Power Model (Non-Gated Clocks) - 3DMark06-HDR1

\subsubsection{Memory}

Memory or DRAM power consumption is one of the more variable subsystems. Similar to the CPU, the application of various power management features yields a wide range of power consumption. For example consider the standard deviation of power consumption in SPECjbb of $1.096 \mathrm{~W}$ compared to average its average of $1.71 \mathrm{~W}$. This variation is caused by the three modes of operation: self-refresh, precharge power down and active. Self-refresh represents the lowest power state in which DRAM contents are maintained by on-chip refresh logic. This mode has a high entry/exit latency and is only entered when the system is expected to be idle for a long period. The memory controller selects this mode as part of its hardware-controlled $\mathrm{C}_{1 \mathrm{e}}$ idle [Bk09] state. Since this state is entered in conjunction with the hypertransport link disconnect, the power savings can be represented using the LinkActive\% metric. Pre-charge power down is a higher-power, lower-latency alternative which provides power savings for short idle phases. This allows pre-charge power savings to be considered with normal DRAM activity power. 
Light activity yields higher precharge residency. DRAM activity is estimated using the DCTAccess metric. The sum of all DCT accesses on both channels (hit, miss and conflict) correlates positively to active DRAM power and negatively to precharge power savings. In most workloads this approach gave error of less than $10 \%$. The two outliers were the CPU subtests of 3DMark06. Due to many of the memory transactions being spaced at intervals just slightly shorter than the precharge power down entry time, the model underestimates power by a larger margin. Higher accuracy would require a direct measurement of pre-charge power down residency or temporal locality of memory transactions. An example of model versus measured Memory power for SYSmark 20073D is provided in Figure 5.8. The modeled power as a function of the DRAM channel access rate (DCTAccess/sec) and Hypertransport activity percentage (LinkActive\%) is given in Equation 5.8. Additional details for the equation components, DCTAccess/sec and LinkActive percent are given in section 5.3.3.

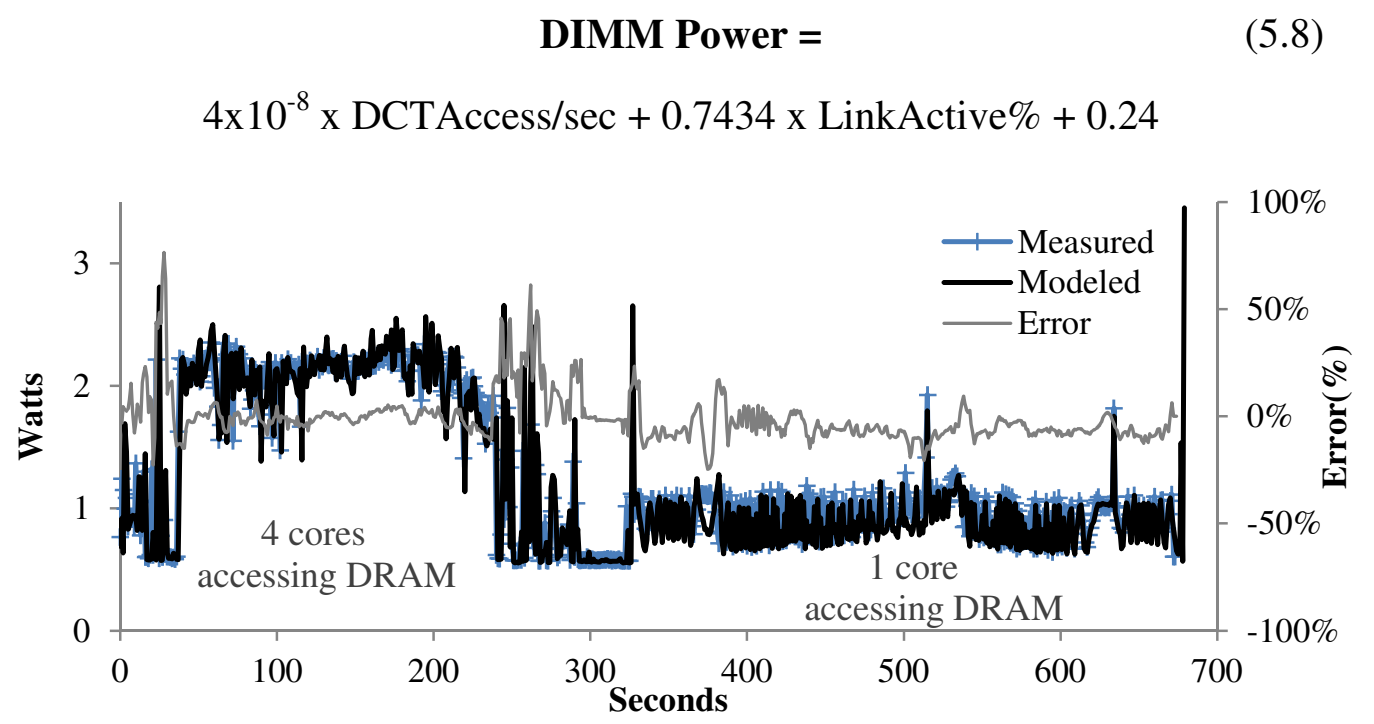

Figure 5.8 DRAM Power Model ( $\sum$ DCT Access, LinkActive) - SYSmark 2007-3D 97 


\subsubsection{Memory Controller}

Since the memory controller is responsible to entry and exit of power saving modes for itself and memory, the memory metrics can also be used to estimate memory controller power. Though both use link active and DCTAccesses the relative weights are different. Memory power has a large sensitivity to the transaction rate, $4 \cdot 10^{-8} \mathrm{~W} /$ transaction/sec. Compare this to the memory controller which is more than four times smaller at $9 \cdot 10^{-9}$ W/transaction/sec. Similarly, transaction-independent portion is much higher for the memory controller at $1.9 \mathrm{~W}$ compared to $0.98 \mathrm{~W}$ for memory. This reflects the unmanaged power consumers in the memory controller. The same 3DMark06 error outliers exist here. An example of model versus measured Memory Controller power for 3DMark06-HDR1 is provided in Figure 5.9. The modeled power is provided below in Equation 5.9.

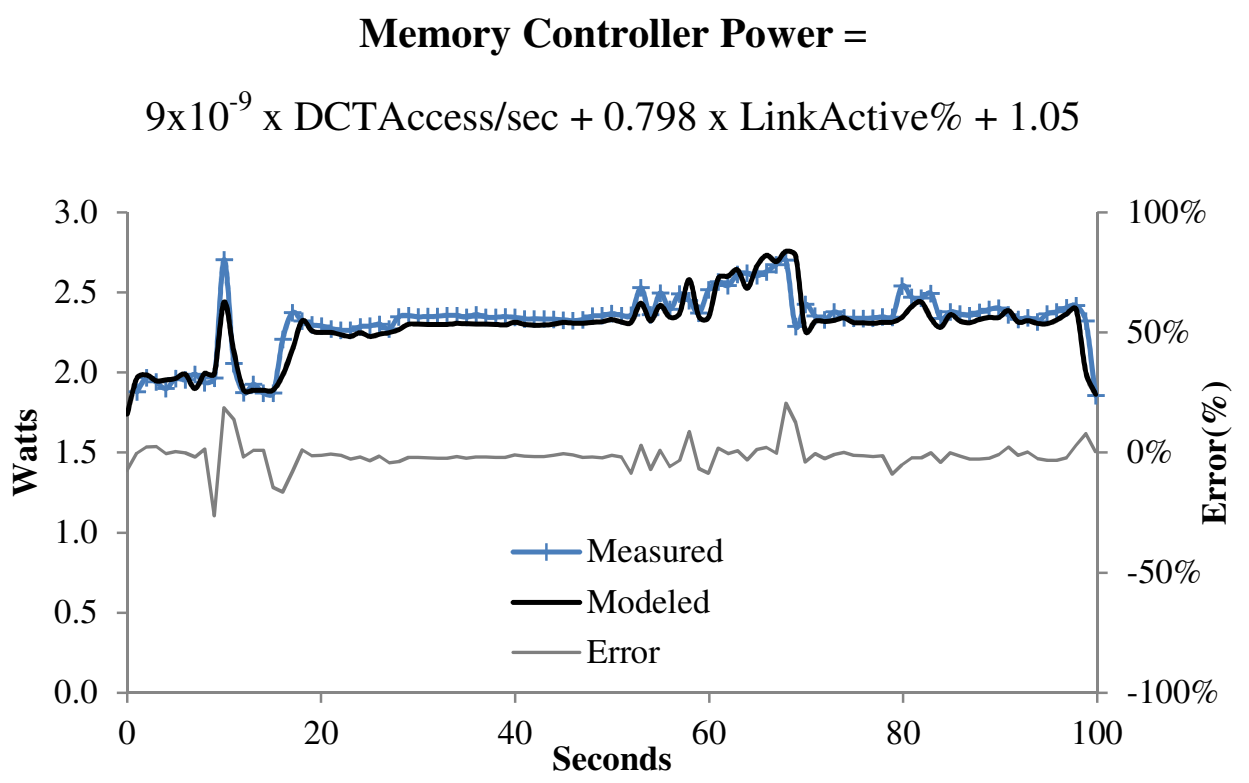

Figure 5.9 Memory Controller Power ( $\sum$ DCT Access, LinkActive) - HDR1 


\subsubsection{Chipset}

The Chipset power model represents power consumed in the Hypertransport controller. Like the memory and memory controller subsystems, power consumption is dominated by the link disconnect state and memory controller accesses. Overall and worst-case were less than the others due to the workload independent contributions being relatively the largest. The model for I/O controller power is shown in Equation 5.10.

$$
\begin{gathered}
\text { I/O Controller Power }= \\
-10^{-16} \times(\text { DCTAcc/sec })^{2}+2 \times 10^{-8} \times(\text { DCTAcc/sec })+1.24 \times \text { LinkAct } \%+1.34
\end{gathered}
$$

\subsubsection{Disk}

The improvements in power management for hard disks between the server-class used in the Server study study [BiJo06-1] and the more recent desktop/mobile disk used here is evident in the power model in Equation 5.11. Rather than the negligible power variation previously observed $(<1 \%)$, the variable portion (one standard deviation) is on average 30\%. This provides more power savings, but a more difficult modeling challenge. As a result average error is higher at $6.6 \%$. The hard disk power model which is a function of CPU-to-I/O transactions and spindle activity is shown in Equation 5.11.

$$
\begin{gathered}
\text { Hard Disk Power }= \\
3 \times 10^{-5} \times(\text { CPUToIOTrans/sec })+0.629 \times \text { SpindleActive }+0.498
\end{gathered}
$$

\subsubsection{Model Validation}

Table 5.5 summarizes the average error results for the six subsystem power models. The CPU subsystem had the second lowest error at $1.64 \%$ largely due to the comprehensive 
power model that was used. Compare to the server power model which averaged over $6 \%$ error using only three inputs. More importantly, this low error rate suggests that performance counter power models are effective across multiple microprocessor architecture generations, platforms, and manufacturers (Intel and AMD).

The chipset power model is also improved compared to the server chipset model with average error of $3.3 \%$. Like the server model, the desktop model contained a large workload-independent component: although in this case it contributed less than half the total chipset power rather than the $100 \%$ seen in the server model.

The memory and memory controller power models had the highest average error with $5.3 \%$ and $6.0 \%$ respectively. The high error is largely due to the CPU portion of the 3DMark06 workload. This workload generates memory transactions at an interval that prevents effective utilization of precharge power down modes. Therefore, the model tends to underestimate memory power consumption. To resolve this error, a metric of typical memory bus idle duration or power down residency would be needed.

The GPU power model had the lowest error rate at slightly less than $1 \%$. This illustrates the effectiveness of the non-gated GPU clocks as a proxy for GPU power. In most workloads the GPU power has a clear bimodal characteristic. Active regions have a power level that is consistent. Idle regions also have a consistent power level due to the presence of idle clock gating. It is expected that as finer grain power management is 
applied to the GPU core logic, larger active power variation will occur. This will necessitate a comprehensive power model such as that used in the CPU.

Table 5.5 Average Error

\begin{tabular}{|l|l|l|l|l|l|l|}
\hline & CPU & Chipset & Memory & Memory Controller & GPU & Disk \\
\hline Idle & $0.3 \%$ & $1.8 \%$ & $1.2 \%$ & $0.4 \%$ & $1.7 \%$ & $2.5 \%$ \\
\hline SPEC CPU2006 Integer & $1.3 \%$ & $4.0 \%$ & $2.3 \%$ & $3.4 \%$ & $0.2 \%$ & $5.3 \%$ \\
\hline SPEC CPU2006 FP & $1.1 \%$ & $2.6 \%$ & $7.2 \%$ & $2.9 \%$ & $0.5 \%$ & $4.4 \%$ \\
\hline gt1 & $0.8 \%$ & $5.3 \%$ & $3.3 \%$ & $1.3 \%$ & $0.9 \%$ & $7.1 \%$ \\
\hline gt2 & $1.2 \%$ & $5.8 \%$ & $3.3 \%$ & $4.4 \%$ & $0.4 \%$ & $8.7 \%$ \\
\hline cpu1 & $1.6 \%$ & $1.8 \%$ & $12.5 \%$ & $14.1 \%$ & $1.0 \%$ & $6.9 \%$ \\
\hline cpu2 & $1.9 \%$ & $1.9 \%$ & $11.5 \%$ & $13.4 \%$ & $1.3 \%$ & $9.2 \%$ \\
\hline hdr1 & $2.2 \%$ & $2.7 \%$ & $0.8 \%$ & $0.7 \%$ & $1.0 \%$ & $0.9 \%$ \\
\hline hdr2 & $1.9 \%$ & $4.7 \%$ & $1.6 \%$ & $8.6 \%$ & $0.7 \%$ & $2.7 \%$ \\
\hline EL & $2.7 \%$ & $9.3 \%$ & $8.5 \%$ & $7.7 \%$ & $0.0 \%$ & $1.8 \%$ \\
\hline VC & $1.0 \%$ & $1.8 \%$ & $8.4 \%$ & $11.7 \%$ & $1.6 \%$ & $10.6 \%$ \\
\hline PR & $2.5 \%$ & $1.1 \%$ & $5.7 \%$ & $5.6 \%$ & $0.9 \%$ & $12.1 \%$ \\
\hline 3D & $2.8 \%$ & $3.5 \%$ & $5.5 \%$ & $4.7 \%$ & $0.0 \%$ & $10.7 \%$ \\
\hline SPECjbb & $1.5 \%$ & $0.4 \%$ & $2.0 \%$ & $4.1 \%$ & $0.8 \%$ & $9.8 \%$ \\
\hline Average & $1.63 \%$ & $3.34 \%$ & $5.27 \%$ & $5.93 \%$ & $0.79 \%$ & $6.62 \%$ \\
\hline
\end{tabular}

Finally, the disk subsystem is the one subsystem which has a higher error rate compared the server power model. In this case the error can be attributed to the effectiveness of ondisk and link power management. In the case of the server model, no active power management is provided. This allows for an accurate model as the workload independent portion dominates. In contrast the more recent desktop hard drive has a workload dependent portion which contributes as much as $1 / 3$ of total power. This causes modeling error to have a larger impact. Note that the subtests with the highest errors are also those with the highest disk utilization. 


\subsection{Summary}

In this section feasibility of predicting complete system power consumption using processor performance events is demonstrated. The models take advantage of the trickledown effect of these events. These events which are visible in the processing unit, are highly correlated to power consumption in subsystems including memory, chipset, I/O, disk and microprocessor. Subsystems farther away from the microprocessor require events more directly related to the subsystem, such as $\mathrm{I} / \mathrm{O}$ device interrupts or clock gating status. Memory models must take into account activity that does not originate in the microprocessor. In this case, DMA events are shown to have a significant relation to memory power. It is shown that complete system power can be estimated with an average error of less than $9 \%$ for each subsystem using performance events that trickle down from the processing unit.

The trickle-down approach is shown to be effective across system architectures, manufacturers and time. High accuracy is achieved on systems from both major PC designers (Intel and AMD), Server and desktop architectures, and across time with systems from 2005 and 2010 exhibiting comparable accuracy. 


\section{Chapter 6 Performance Effects of}

\section{Dynamic Power Management}

\subsection{Direct and Indirect Performance Impacts}

\subsubsection{Transition Costs}

Due to physical limitations, transitioning between adaptation states may impose some cost. The cost may be in the form of lost performance or increased energy consumption. In the case of DVFS, frequency increases require execution to halt while voltage supplies ramp up to their new values. This delay is typically proportional to the rate of voltage change (seconds/volt). Frequency decreases typically do not incur this penalty as most digital circuits will operate correctly at higher than required voltages. Depending on implementation, frequency changes may incur delays. If the change requires modifying the frequency of clock generation circuits (phase locked loops), then execution is halted until the circuit locks on to its new frequency. This delay may be avoided if frequency reductions are implemented using methods which maintain a constant frequency in the clock generator. This is the approach used in Quad-Core AMD processor c-state implementation. Delay may also be introduced to limit current transients. If a large number of circuits all transition to a new frequency, then excessive current draw may result. This has a significant effect on reliability. Delays to limit transients are 
proportional to the amount of frequency change (seconds/MHz). Other architecturespecific adaptations may have variable costs per transition. For example, powering down a cache requires modified contents to be flushed to the next higher level of memory. This reduces performance and may increase power consumption due to the additional bus traffic. When a predictive component is powered down it no longer records program behavior. For example, if a branch predictor is powered down during a phase in which poor predictability is expected, then branch behavior is not recorded. If the phase actually contains predictable behavior, then performance may be lost and efficiency may be lost. If a unit is powered on and off in excess of the actual program demand, then power and performance may be significantly affected by the flush and warm-up cycles of the components. In this study the focus is on fixed cost per transition effects such as those required for voltage and frequency changes.

\subsubsection{Workload Phase and Policy Costs}

In the ideal case the transition costs described above do not impact performance and save maximum power. The reality is that performance of dynamic adaption is greatly affected by the nature of workload phases and the power manager's policies. Adaptations provide power savings by setting performance to the minimum level required by the workload. If the performance demand of a workload were known in advance, then setting performance levels would be trivial. Since they are not known, the policy manager must estimate future demand based on the past. Existing power managers, such as those used in this study (Windows Vista [Vi07] and SLES Linux [PaSt06]), act in a reactive mode. They 
can be considered as predictors which always predict the next phase to be the same as the last. This approach works well if the possible transition frequency up the adaptation is greater than the phase transition frequency of workload. Also, the cost of each transition must be low considering the frequency of transitions. In real systems, these requirements cannot currently be met. Therefore, the use of power adaptations does reduce performance to varying degrees depending on workload. The cost of mispredicting performance demand is summarized below.

Underestimate: Setting performance capacity lower than the optimal value causes reduced performance. Setting performance capacity lower than the optimal value may cause increased energy consumption due to increased runtime. It is most pronounced when the processing element has effective idle power reduction.

Overestimate: Setting performance capacity higher than the optimal value reduces efficiency as execution time is not reduced yet power consumption is increased. This case is common in memory-bound workloads.

Optimization Points: The optimal configuration may be different depending on which characteristic is being optimized. For example, Energy $\times$ Delay may have a different optimal point compared to Energy $\times$ Delay $^{2}$.

\subsubsection{Performance Effects}

P-states and C-states impact performance in two ways: Indirect and Direct. Indirect performance effects are due to the interaction between active and idle cores. In the case 
of Quad-Core AMD processors, this is the dominant effect. When an active core performs a cache probe of an idle core, latency is increased compared to probing an active core. The performance loss can be significant for memory-bound (cache probeintensive) workloads. Direct performance effects are due to the current operating frequency of an active core. The effect tends to be less compared to indirect, since operating systems are reasonably effective at matching current operating frequency to performance demand. These effects are illustrated in Figure 6.1.

Two extremes of workloads are presented: the compute-bound crafty and the memorybound equake. For each workload, two cases are presented: fixed and normal scheduling. Fixed scheduling isolates indirect performance loss by eliminating the effect of OS frequency scheduling and thread migration. This is accomplished by forcing the software thread to a particular core for the duration of the experiment. In this case, the thread runs always run at the maximum frequency. The idle cores always run at the minimum frequency. As a result, crafty achieves 100 percent of the performance of processor that does not use dynamic power management. In contrast, the memory-bound equake shows significant performance loss due to the reduced performance of idle cores. Direct performance loss is shown in the dark solid and light solid lines, which utilize OS scheduling of frequency and threads. Because direct performance losses are caused by suboptimal frequency in active cores, the compute-bound crafty shows a significant performance loss. The memory-bound equake actually shows a performance 
improvement for low idle core frequencies. This is caused by idle cores remaining at a high frequency following a transition from active to idle.

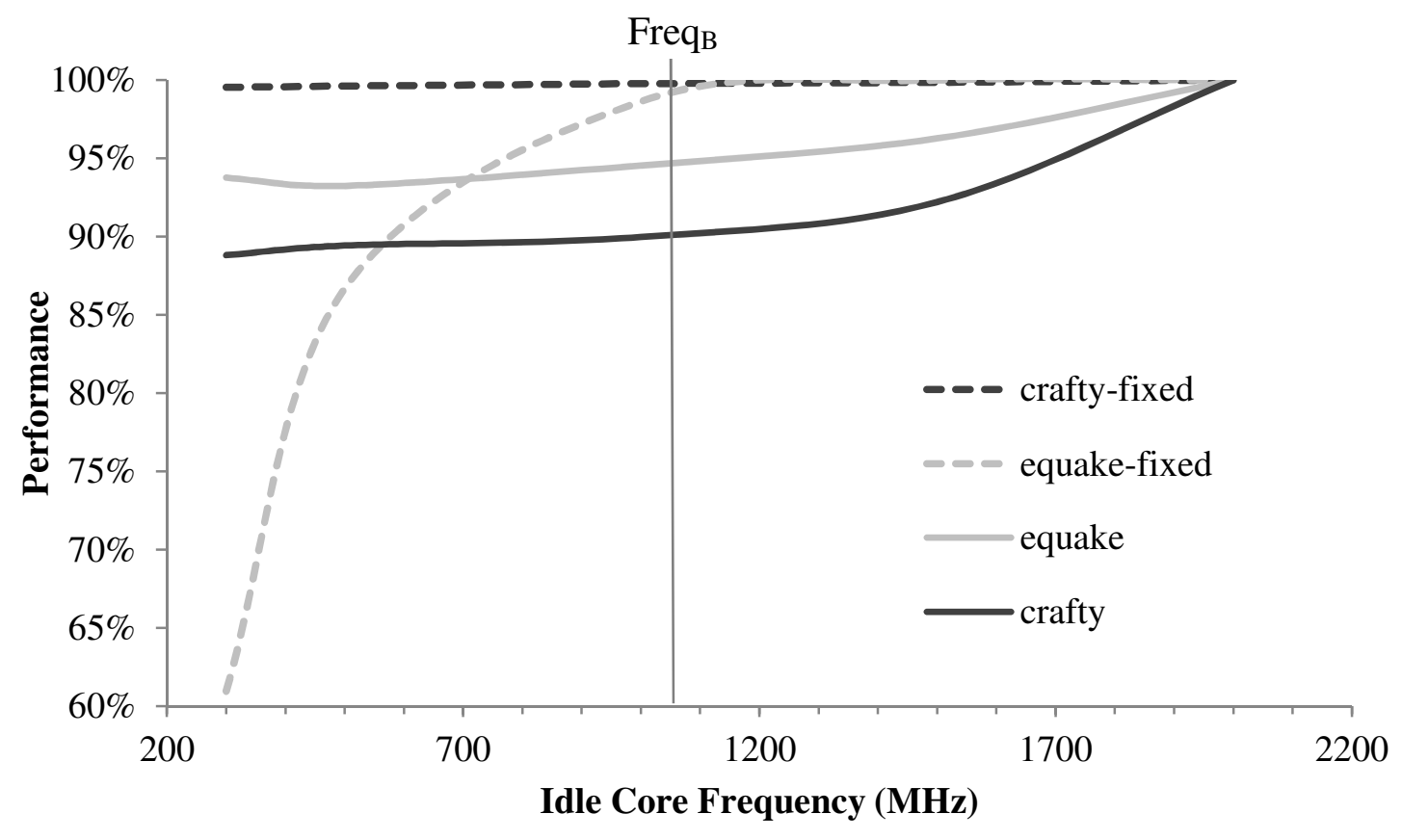

Figure 6.1 Direct and Indirect Performance Impact

\subsubsection{Indirect Performance Effects}

The amount of indirect performance loss is mostly dependent on the following three factors: Idle core frequency, OS p-state transition characteristics, and OS scheduling characteristics. The probe latency (time to respond to probe) is largely independent of idle core frequency above the "breakover" frequency (Freq $\left.{ }_{B}\right)$. Below Freq ${ }_{B}$ the performance drops rapidly at an approximately linear rate. This can be seen in Figure 6.1 as the dashed light line. The value of Freq ${ }_{B}$ is primarily dependent on the inherent probe latency of the processor and the number of active and idle cores. Increasing the active core frequency increases the demand for probes and therefore increases Freq $q_{\mathrm{B}}$. Increasing 
the number of cores has the same effect. Therefore, multi-socket systems tend to have a higher Freq $\mathrm{B}_{\mathrm{B}}$. Assuming at least one idle core, the performance loss increases as the ratio of active-to-idle cores increases. For an N-core processor, the worst-case is N-1 active cores with 1 idle core. To reduce indirect performance loss, the system should be configured to guarantee than the minimum frequency of idle cores is greater than or equal to Freq ${ }_{\mathrm{B}}$. To this end the Quad-Core AMD processor uses clock ramping in response to probes [Bk09]. When an idle core receives a probe from an active core, the idle core frequency is ramped to the last requested p-state frequency. Therefore, probe response performance is dictated by the minimum idle core frequency supported by the processor. For the majority of workloads, this configuration yield less than 10 percent performance loss due to idle core probe latency.

The other factors in indirect performance loss are due to the operating system interaction with power management. These factors, which include OS p-state transition and scheduling characteristics, tend to mask the indirect performance loss. Ideally, the OS selects a high frequency p-state for active cores and a low frequency for idle cores. However, erratic workloads (many phase transitions) tend to cause high error rates in the selection of optimal frequency. Scheduling characteristics that favor load-balancing over processor affinity worsen the problem. Each time the OS moves a process from one core to another, a new phase transition has effectively been introduced. More details of OS pstate transitions and scheduling characteristics are given in the next section on direct performance effects. 


\subsubsection{Direct Performance Effects}

Since the OS specifies the operating frequency of all cores (p-states), the performance loss is dependent on how the OS selects a frequency. To match performance capacity (frequency) to workload performance demand, the OS approximates demand by counting the amount of slack time a core has. For example, if a core runs for only $5 \mathrm{~ms}$ of its 10 ms time allocation it is said to be 50 percent idle. In addition to the performance demand information, the OS p-state algorithm uses a form of low-pass filtering, hysteresis, and performance estimation/bias to select an appropriate frequency. These characteristics are intended to prevent excessive p-state transitions. This has been important historically since transitions tended to cause a large performance loss (PLL settling time, VDD stabilization). However, in the case of Quad-Core AMD processors and other recent designs, the p-state transition times have been reduced significantly. As a result, this approach may actually reduce performance for some workloads and configurations. See the light, solid line for equake and dark dashed lines for crafty in Figure 6.1. These two cases demonstrate the performance impact of the OS p-state transition hysteresis.

As an example, consider a workload with short compute-bound phases interspersed with similarly short idle phases. Due to the low-pass filter characteristic, the OS does not respond to the short duration phases by changing frequency. Instead, the cores run at reduced frequency with significant performance loss. In the pathologically bad case, the OS switches the frequency just after the completion of each active/idle phase. The cores run at high frequency during idle phases and low frequency in active phases. Power is 
increased while performance is decreased. OS scheduling characteristics exacerbate this problem. Unless the user makes use of explicit process affinity or an affinity library, some operating systems will attempt to balance the workloads across all cores. This causes a process to spend less contiguous time on a particular core. At each migration from one core to another there is a lag from when the core goes active to when the active core has its frequency increased. The aggressiveness of the p-state setting amplifies the performance loss/power increase due to this phenomenon. Fortunately, recent operating systems such as Microsoft Windows Vista provide means for system designers and end users to adjust the settings to match their workloads/hardware [Vi07].

\subsection{Reactive Power Management}

In this section, results are presented to show the effect that dynamic adaptations ultimately have on performance and power consumption. All results are obtained from a real system, instrumented for power measurement. The two major areas presented are probe sensitivity (indirect) and operating system effects (direct).

First, the probe sensitivity of SPEC CPU2006 is considered. Table 6.1 shows performance loss due to the use of p-states. In this experiment the minimum $\mathrm{p}$-state is set below the recommended performance breakover point for probe response. This emphasizes the inherent sensitivity workloads have to probe response. Operating system frequency scheduling is biased towards performance by fixing active cores at the maximum frequency and idle cores at the minimum frequency. These results suggest that floating point workloads tend to be most sensitive to probe latency. However, in the case 
of SPEC CPU2000 workloads, almost no performance loss is shown. The reason, as shown in section 4.3.1, is that smaller working set size reduces memory traffic and, therefore, the dependence on probe latency. For these workloads only swim, equake, and eon showed a measureable performance loss.

Table 6.1 Performance Loss Due to Low Idle Core Frequency - SPEC CPU 2006

\begin{tabular}{|l|l|l|l|}
\hline \multicolumn{4}{|l|}{ SPEC CPU $2006-$ INT } \\
\hline perlbench & $-0.8 \%$ & sjeng & $0.0 \%$ \\
\hline bzip2 & $-1.0 \%$ & libquantum & $-7.0 \%$ \\
\hline gcc & $-3.6 \%$ & h264ref & $-0.8 \%$ \\
\hline mcf & $-1.8 \%$ & omnetpp & $-3.7 \%$ \\
\hline gobmk & $-0.3 \%$ & astar & $-0.5 \%$ \\
\hline hmmer & $-0.2 \%$ & \multicolumn{3}{l|}{} \\
\hline SPEC CPU $2006-\mathrm{FP}$ & \multicolumn{5}{|l|}{} \\
\hline bwaves & $-5.6 \%$ & soplex & $-6.7 \%$ \\
\hline games & $-0.6 \%$ & povray & $-0.5 \%$ \\
\hline milc & $-7.9 \%$ & calculix & $-0.6 \%$ \\
\hline zeusmp & $-2.1 \%$ & GemsFDTD & $-5.9 \%$ \\
\hline gromacs & $-0.3 \%$ & tonto & $-0.6 \%$ \\
\hline cactusADM & $-2.6 \%$ & lbm & $-5.6 \%$ \\
\hline leslie3D & $-6.0 \%$ & wrf & $-3.2 \%$ \\
\hline namd & $-0.1 \%$ & sphinx3 & $-5.6 \%$ \\
\hline dealII & $-1.3 \%$ & \multicolumn{2}{|l}{} \\
\hline
\end{tabular}

Next, it is shown that by slightly increasing the minimum p-state frequency it is possible to recover almost the entire performance loss. Figure 6.2 shows an experiment using a synthetic kernel with high probe sensitivity with locally and remotely allocated memory. The remote case simply shows that the performance penalty of accessing remote memory can obfuscate the performance impact of minimum p-state frequency. The indirect performance effect can be seen clearly by noting that performance increases rapidly as the idle core frequency is increased from $800 \mathrm{MHz}$ to approximately $1.1 \mathrm{GHz}$. This is a 
critical observation since the increase in power for going from $800 \mathrm{MHz}$ to $1.1 \mathrm{GHz}$ is much smaller than the increase in performance. The major cause is that static power represents a large portion of total power consumption. Since voltage dependence exists between all cores in a package, power is only saved through the frequency reduction. There is no possibility to reduce static power since voltage is not decreased on the idle cores.

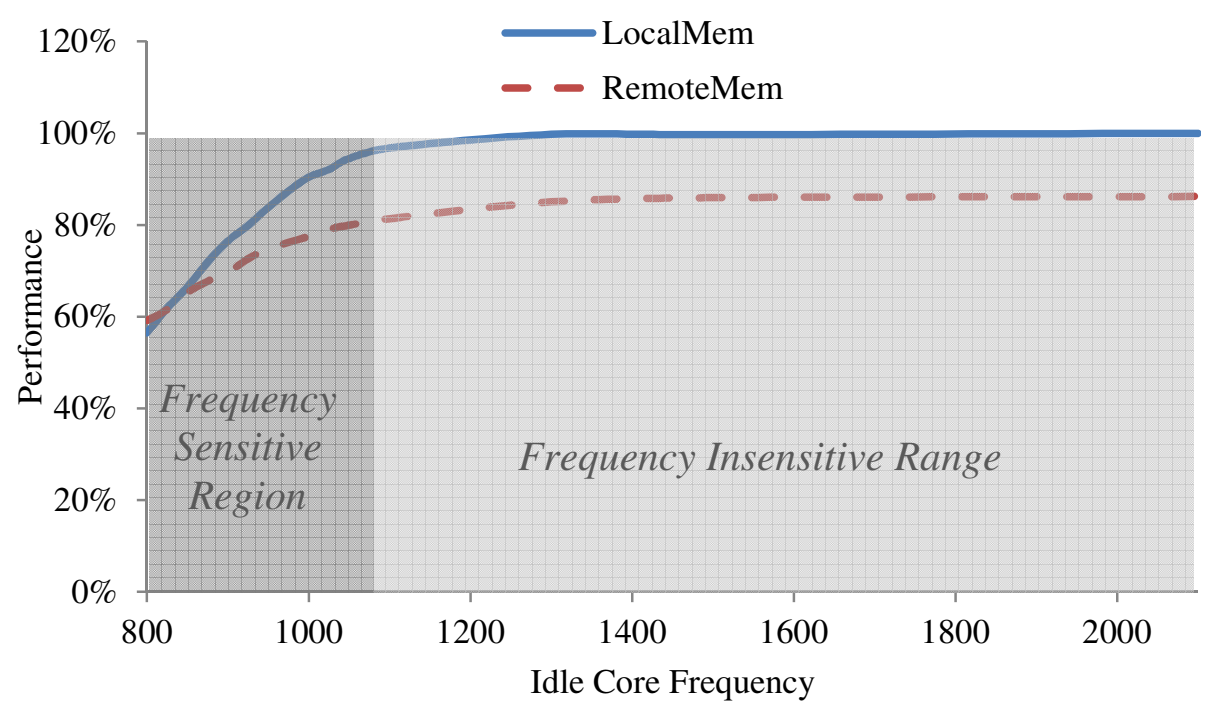

Figure 6.2 Remote and Local Probe Sensitivity

Using the same synthetic kernel the effect of p-states is isolated from c-states. Since the p-state experiments show that indirect performance loss is significant below the breakover point, now c-state settings that do not impose the performance loss are considered. To eliminate the effect of this performance loss the processor can be configured for ramped probe response. In this mode, idle cores increase their frequency before responding to probe requests. To obtain an optimal tradeoff between performance and power settings, this setting mode can be modulated using hysteresis, implemented by 
adjusting a hysteresis timer. The timer specifies how long the processor remains at the increased frequency before returning to the power saving mode. The results are shown in Figure 6.3. The blue line represents the performance loss due to slow idle cores caused by the application of c-states only. Like the p-state experiments, performance loss reaches a clear breakpoint. In this case, the breakover point represents 40 percent of the maximum architected delay. Coupling c-states with p-states, the red shows that the breakover point is not as distinct since significant performance loss already occurs. Also, like the p-state experiments, setting the hysteresis timer to a value of the breakover point increases performance significantly while increasing power consumption only slightly.

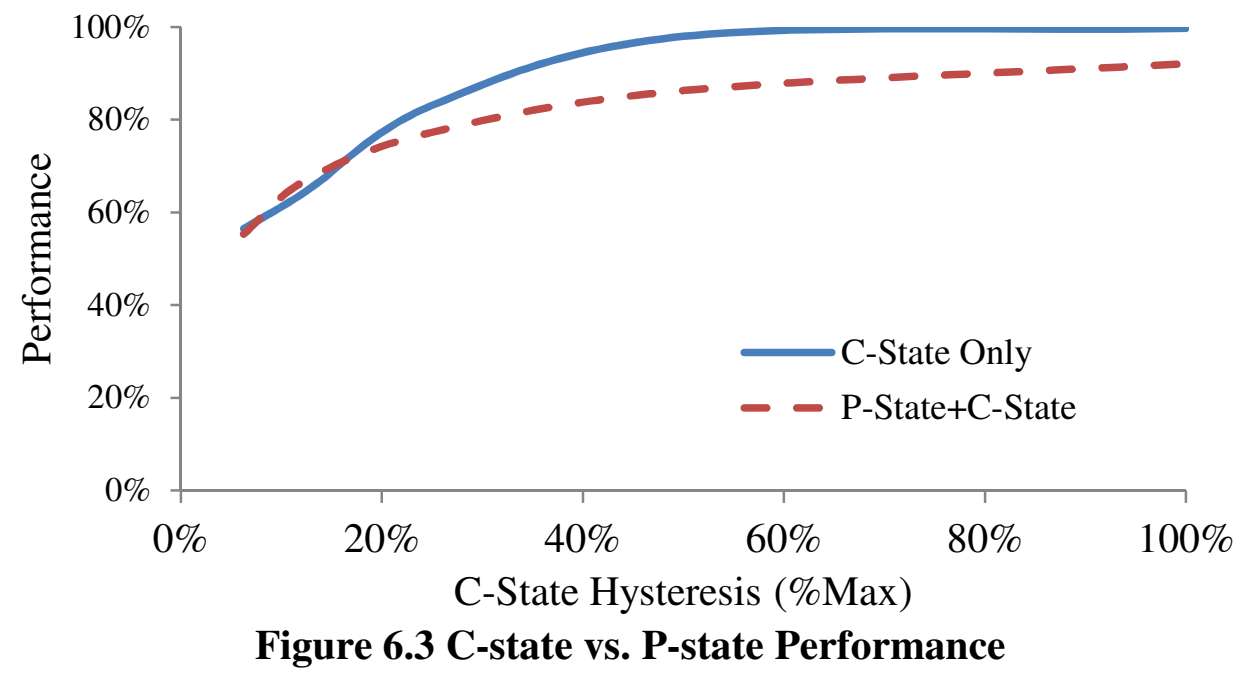

Next, the effect of operating system tuning parameters for power adaptation selection is considered. In order to demonstrate the impact of slow p-state selection, Figure 6.4 is presented. The effect is shown by varying a single OS parameter while running the SYSmark E-Learning subtest. In this graph, the TimeCheck value is varied from $1 \mathrm{~ms}$ to $1000 \mathrm{~ms}$. TimeCheck controls how often the operating system will consider a p-state 
change. Two major issues were found: minimum OS scheduling quanta and increase/decrease filter.

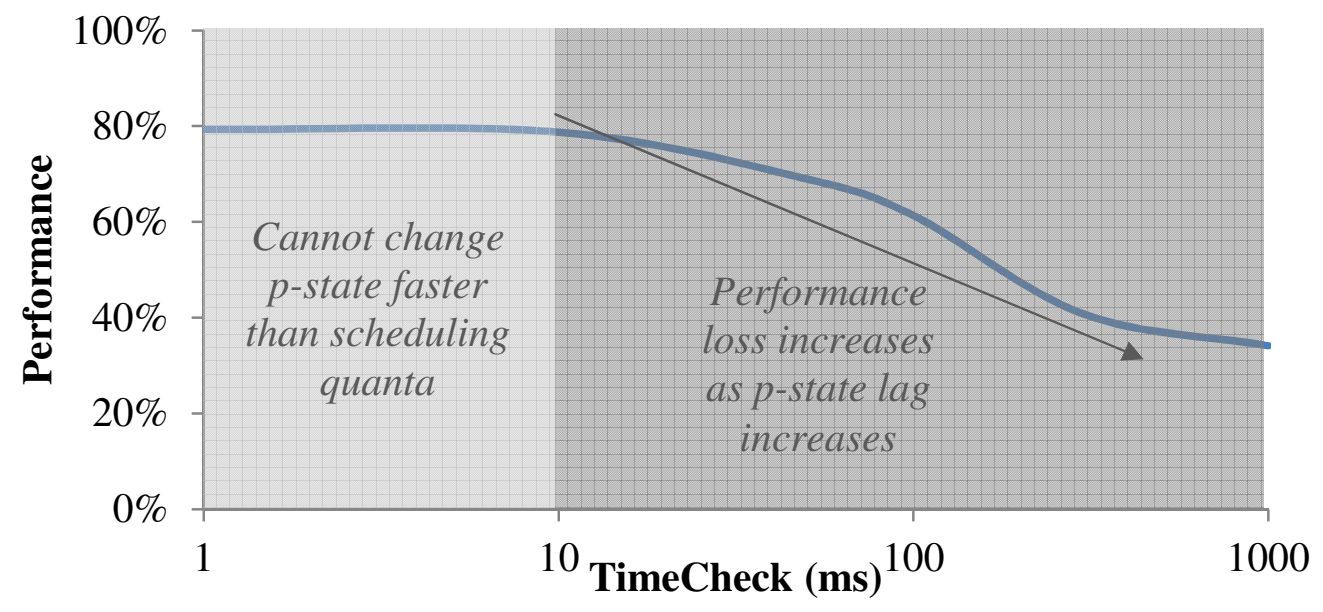

Figure 6.4 Varying OS P-state Transition Rates

First, performance remains constant when scaling from 1 us to $10 \mathrm{~ms}(<1 \mathrm{~ms}$ not depicted). This is attributable to the OS implementation of scheduling. For Microsoft Windows Vista, all processes are scheduled on the $10 \mathrm{~ms}$ timer interrupt. Setting TimeCheck to values less than $10 \mathrm{~ms}$ will have no impact since p-state changes, like all process scheduling, occur only on 10-ms boundaries. Second, even at the minimum TimeCheck value, performance loss is at 80 percent. The reason is that other settings become dominant below $10 \mathrm{~ms}$. In order for a p-state transition to occur the workload must overcome the in-built low-pass filter. This filter is implemented as a combination of two thresholds: increase/decrease percent and increase/decrease time. The percent threshold represents the utilization level that must be crossed in order to consider a pstate change. The threshold must be exceeded for a fixed amount of time specified by 
increase/decrease time. Since the increase time is much longer than TimeCheck $(300 \mathrm{~ms}$ vs. $10 \mathrm{~ms}$ ), significant performance is lost even at the minimum setting.

To reduce the impact of slow p-state transitions OS settings are selected that increase transition rates. In a general sense, frequent $\mathrm{p}$-state transitions are not recommended due to the hardware transition costs. However, these experiments have shown that the performance cost for slow OS-directed transitions is much greater than that due to hardware. This can be attributed to the relatively fast hardware transitions possible on Quad-Core AMD processors. Compared to OS transitions which occur at $10 \mathrm{~ms}$ intervals, worst-case hardware transitions occur in a matter of 100's of microseconds. Figure 6.5 shows the effect of optimizing p-state changes to the fastest rate of once every $10 \mathrm{~ms}$. The probe-sensitive equake is shown with and without "fast p-states." This approach yields between 2 percent and 4 percent performance improvement across the range of useful idle core frequencies. As is shown in the next section, this also improves power savings by reducing active-to-idle transition times.

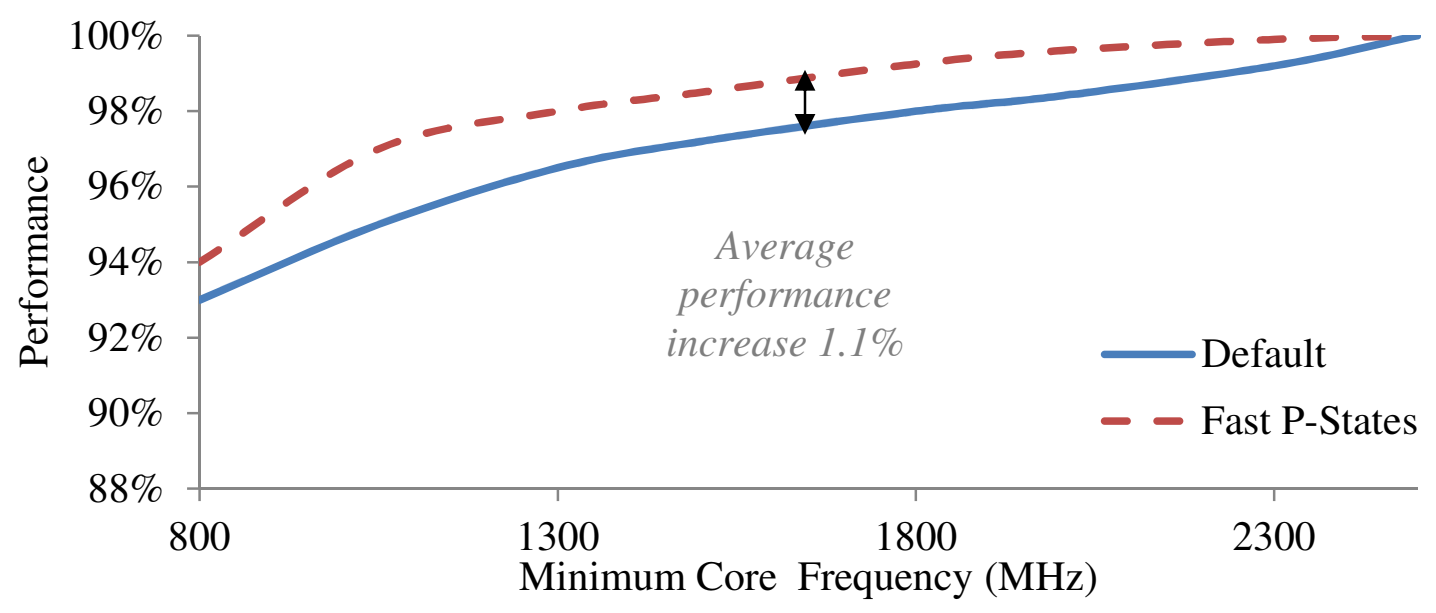

Figure 6.5 Effect of Increasing P-state Transition Rate

115 


\subsubsection{Power and Performance Results}

In this section results for $\mathrm{p}$-state and c-state settings are presented that reflect the findings of the previous sections. In this case the Microsoft Windows Vista operating system running desktop workloads is studied. This approach gives the highest exposure to the effect the operating system has on dynamic adaptations. By choosing desktop workloads, the number of phase transitions and, therefore, OS interaction is increased. Since these workloads model user input and think times, idle phases are introduced. These idle phases are required for OS study since the OS makes use of idle time for selecting the operating point. Also, Microsoft Windows Vista exposes tuning parameters to scale the built-in adaptation selection algorithms for power savings versus performance. Table 6.2 shows power and performance results for SYSmark 2007 using a range of settings chosen based on the results of the previous sections.

Table 6.2 Power/Performance Study: SYSmark 2007

\begin{tabular}{|c|c|c|c|}
\hline Workload & P-State Selection & Performance Loss & Power Savings \\
\hline E-Learning & Default & $8.8 \%$ & $43.1 \%$ \\
\hline Video Creation & Default & $6.2 \%$ & $44.7 \%$ \\
\hline Productivity & Default & $9.5 \%$ & $45.3 \%$ \\
\hline 3D & Default & $5.9 \%$ & $45.9 \%$ \\
\hline E-Learning & Fast & $6.4 \%$ & $45.9 \%$ \\
\hline Video Creation & Fast & $5.2 \%$ & $46.1 \%$ \\
\hline Productivity & Fast & $8.0 \%$ & $47.8 \%$ \\
\hline 3D & Fast & $4.6 \%$ & $48.2 \%$ \\
\hline E-Learning & Fast-Perf & $1.5 \%$ & $32.9 \%$ \\
\hline Video Creation & Fast-Perf & $1.8 \%$ & $25.4 \%$ \\
\hline Productivity & Fast-Perf & $2.5 \%$ & $27.9 \%$ \\
\hline 3D & Fast-Perf & $1.4 \%$ & $35.1 \%$ \\
\hline
\end{tabular}


In order to reduce p-state performance loss, the idle core frequency is set to $1250 \mathrm{MHz}$. To prevent c-state performance loss, ramped probe mode is used with the hysteresis time set above the breakover point. Also, $\mathrm{C}_{1 \mathrm{e}}$ mode is disabled to prevent obscuring the idle power savings of the architected p-states and c-states. The $\mathrm{C}_{1 \mathrm{e}}$ state is a microarchitectural feature that reduces power when all cores are idle. The power and performance effects of this state can reduce the measureable effect of the p-state and cstate decisions made by the operating system.

Two important findings are made regarding adaption settings. First, setting power adaptations in consideration of performance bottlenecks reduces performance loss while retaining power savings. Second, reducing OS p-state transition time increases performance and power savings. Table 6.2 shows the resultant power and performance for a range of OS p-state algorithm settings. It is shown that performance loss can be limited to less than 10 percent for any individual subtest while power savings average 45 percent compared to not using power adaptations. The effect of workload characteristics is evident in the results. E-learning and productivity show the greatest power savings due to their low utilization levels. These workloads frequently use only a single core. At the other extreme, 3D and video creation have less power savings and a greater dependence on adaption levels. This indicates that more parallel workloads have less potential benefit from p-state and c-state settings, since most cores are rarely idle. For those workloads, idle power consumption is more critical. These results also point out the limitation of 
existing power adaptation algorithms. Since current implementations only consider idle time rather than memory-boundedness, the benefit of p-states is underutilized.

Additionally, the effect of adjusting operating system p-state transition parameters is shown in Table 6.2. Columns Fast and Fast-Perf represent cases in which p-state transitions occur at the fastest rate and bias towards performance respectively. Since existing operating systems such as Microsoft Windows XP and Linux bias p-state transitions toward performance, these results can be considered representative for those cases.

\subsection{Summary}

In this section, a power and performance analysis of dynamic power adaptations is presented for a Quad-Core AMD processor. Performance and power are shown to be greatly affected by direct and indirect characteristics. Direct effects are composed of operating system thread and frequency scheduling. Slow transitions by the operating system between idle and active operation cause significant performance loss. The effect is greater for compute-bound workloads which would otherwise be unaffected by power adaptations. Slow active-to-idle transitions also cause reduced power savings. Indirect effects due to shared, power-managed resources such as caches can greatly reduce performance if idle core frequency reductions are not limited sufficiently. These effects are more pronounced in memory-bound workloads since performance is directly related to accessing shared resources between the active and idle cores. Finally, it is shown that 
performance loss and power consumption can be minimized through careful selection of hardware adaptation and software control parameters. In the case of Microsoft Windows Vista running desktop workloads, performance loss using a naïve operating system configuration is less than 8 percent on average for all workloads while saving an average of 45 percent power. Using an optimized operating system configuration, performance loss drops to less than 2 percent with power savings of 30 percent.

While the results attained through optimizing a reactive operating system power adaptation are promising, further improvement can be achieved through new approaches. The existing adaptations algorithms have several limitations including poor response to phase changes and a lack of process awareness and frequency-sensitivity. The ability to increase the responsiveness of the reactive algorithm is limited since excessive adaptations reduce performance and increase energy consumption. To attain higher performance and efficiency, a predictive adaptation is required. Predictive adaptation effectively provides the responsiveness of a maximally reactive scheme without the overhead of excessive adaptation.

Another limitation is the lack of frequency-sensitivity awareness in current algorithms. To make best use of dynamic processor voltage and frequency scaling, the sensitivity of a workload to frequency should be accounted. By knowing the frequency sensitivity, workloads which do not benefit from high frequency could achieve much lower power. Similarly, workloads that scale well with frequency can attain higher performance by avoiding the use of excessively low frequencies. 


\section{Chapter 7 Predictive Power Management}

\subsection{Core-Level Activity Prediction}

Existing power management techniques operate by reducing performance capacity (frequency, voltage, resource size) when performance demand is low, such as at idle or similar low activity phases. In the case of multi-core systems, the performance and power demand is the aggregate demand of all cores in the system. Monitoring aggregate demand makes detection of phase changes difficult (active-to-idle, idle-to-active, etc.) since aggregate phase behavior obscures the underlying phases generated by the workloads on individual cores. This causes sub-optimal power management and overprovisioning of power resources. In this study, these problems are addressed through core-level, activity prediction.

The core-level view makes detection of phase changes more accurate, yielding more opportunities for efficient power management. Due to the difficulty in anticipating activity level changes, existing operating system power management strategies rely on reaction rather than prediction. This causes sub-optimal power and performance since changes in performance capacity by the power manager lag changes in performance demand. To address this problem we propose the Periodic Power Phase Predictor (PPPP). This activity level predictor decreases SYSmark 2007 client/desktop processor power consumption by $5.4 \%$ and increases performance by $3.8 \%$ compared to the 
reactive scheme used in Windows Vista operating system. Applying the predictor to the prediction of processor power, accuracy is improved by $4.8 \%$ compared to a reactive scheme.

Dynamic power management provides a significant opportunity for increasing energy efficiency and performance of computing systems. Energy efficiency is increased by reducing performance capacity (frequency, parallelism, speculation, etc.) when the demand for performance is low. Efficiency or a lack thereof may impact the performance of a system. High-density server and multi-core processor performance is limited by power delivery and dissipation. Reducing the power consumption in one component may allow other components to consume more power (higher voltage and frequency) and therefore achieve higher performance. These systems would otherwise have higher performance if they were not sharing power resources [B107] [McPo06] [FaWe07] [WaCh08] [WuJu05]. Power management allows the performance of cores/systems sharing power to increase by "borrowing" power from idle or performance-insensitive cores/systems and reallocating it to heavily-utilized cores/systems.

The challenge in applying power management to increase efficiency and performance is in identifying when to adapt performance capacity. The ubiquitous, architected solution implemented in operating systems such as Windows/Linux is to react to changes in performance demand. Though this approach is simple, it performs sub-optimally [BiJo08] [DiSo08] for workloads with many distinct and/or short phases. Each time a workload transitions from a phase of low performance demand to a phase of high 
performance demand, reactive power management increases performance capacity sometime after the phase transition. During the time between the change in demand and capacity, performance may be less than optimal. Similarly, power consumption is suboptimal on transitions from high to low demand. The amount of performance loss is proportional to the number of phase changes in the workload and the lag between demand and capacity. For increasing performance in power-limited situations, reactions must be fast to prevent overshooting the power limit or missing opportunities to increase performance.

Identifying when to adapt is complicated by the presence of multiple cores sharing power resources. Consider Figure 7.1. Core-level power consumption is shown for a system with multiple simultaneous threads. The program threads are fixed to the cores with thread $\mathrm{N}$ on core $\mathrm{N}$, thread $\mathrm{N}-1$ on core $\mathrm{N}-1$, etc. Since power monitoring is typically provided at the system-level [Po10], existing power control techniques use the erratic fluctuations in the total power for predicting future behavior. This is unfortunate since in this example, the individual threads have a periodic, easily discernable pattern, while the pattern in the aggregate power is less discernable. If power phases can be tracked at the core-level, accurate dynamic power management schemes can be devised.

To improve the effectiveness of power management the use of predictive, core-level power management is proposed. Rather than reacting to changes in performance demand, past activity patterns are used to predict future transitions. Rather than using aggregate power information, activity and power measured at the core-level is used. 

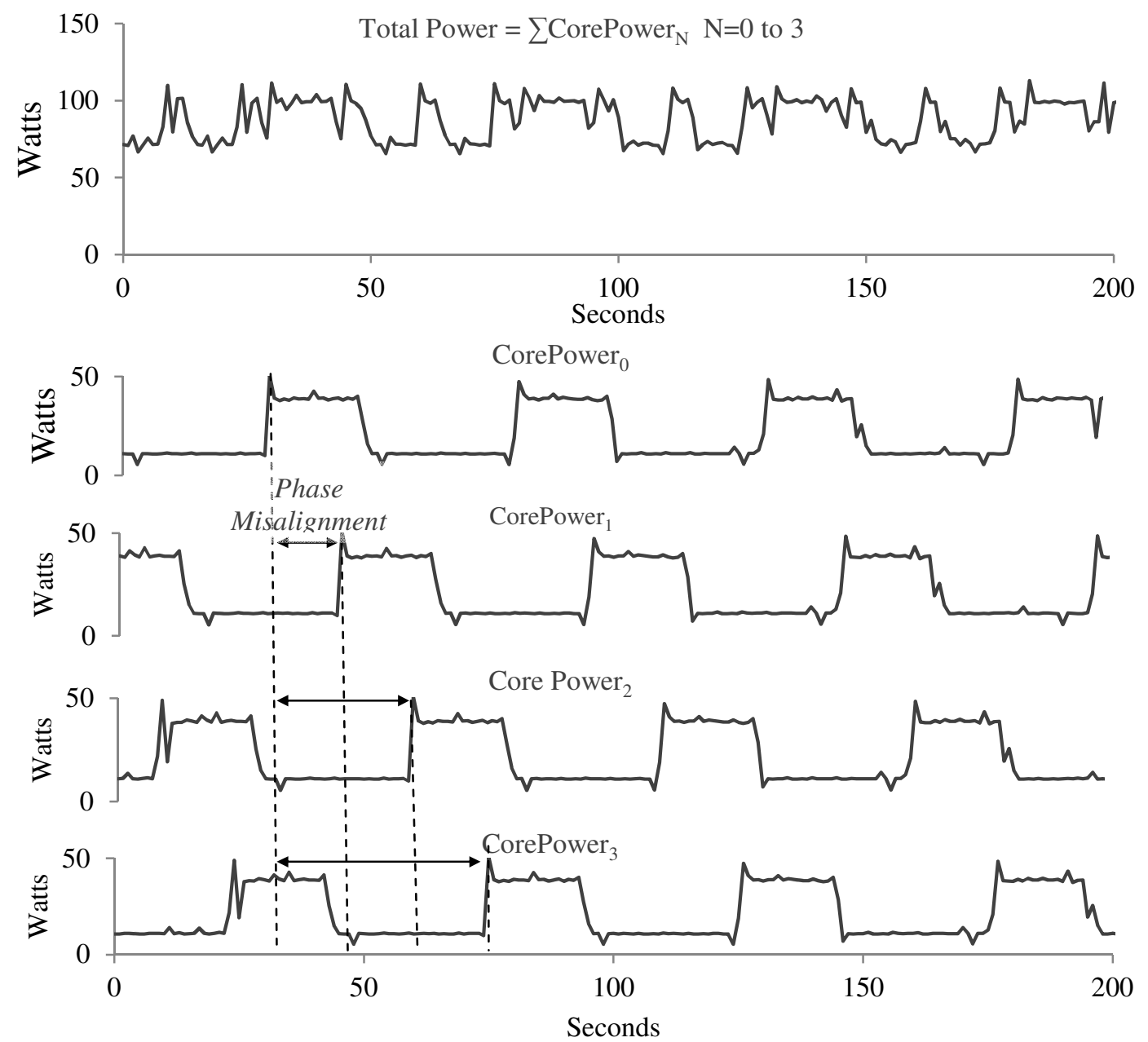

Figure 7.1: Thread and Aggregate Power Patterns

To analyze the effectiveness of the predictor the SYSmark 2007 benchmark is used. It contains numerous, desktop/personal computing applications such as Word, Excel, PowerPoint, Photoshop, Illustrator, etc. The benchmark is structured to have a high degree of program phase transitions, including extensive use of processor idle states (cstates) and performance states (p-states) [Ac07]. Rather than being strictly dominated by the characteristics of the workload itself, SYSmark 2007 accounts for the impact of periodic, operating system scheduling and $\mathrm{I} / \mathrm{O}$ events. Using this observation, we 
construct a periodic power phase predictor. This simple periodic predictor tracks and predicts periodic phases by their duration. By predicting future demand, aggressive adaptations can be applied. The core-level predictive technique outperforms existing reactive power management schemes by reducing the effective lag between workload phase transitions and power management decisions. By predicting the individual power contribution of each thread rather than predicting the aggregate effect, complex phases can be predicted. The contributions of this chapter are summarized as follows.

(1) Concept of core-level power phases in multi-core systems. Core-level power phases unveil more opportunities for power saving adaptations than are possible if only aggregate system level information is used.

(2) A simple, periodic power phase predictor. Though prior research [DuCa03] [IsBu06] demonstrates the effectiveness of power management using predictive schemes on uniprocessors, this research shows its effectiveness when applied to multi-core systems with operating system scheduling interactions. The proposed predictive power management is compared against the reactive algorithm in the Windows Vista operating system. Previous research focused on single-threaded SPEC CPU 2000 benchmarks, while this study uses SYSmark 2007 which includes popular desktop/personal computing applications such as Microsoft Word, Excel, PowerPoint, Adobe Photoshop, Illustrator, etc. These workloads include difficult to predict power management events due to large numbers of interspersed idle phases and frequent thread migrations. 


\subsection{Commercial DVFS Algorithm}

Existing, commercial DVFS algorithms in Windows and Linux operating systems select processor clock frequencies by reacting to changes in core activity level [Vi07]. Activity level represents the ratio of architected code execution (active time) to wall clock time (active + idle time). Processors become idle when they exhaust the available work in their run queues. The intent of these algorithms is to apply low frequencies when a processor is mostly idle and high frequencies when mostly active. This provides high performance when programs can benefit and low power when they cannot.

In this study the predictive DVFS algorithm is compared to that used in the Windows Vista operating system [Vi07]. This Vista algorithm reactively selects DVFS states (core frequency) in order to maintain a target core activity level of 30\%-50\%. See Figure 7.2. The "predicted" activity level is the last observed activity level, hence this is a reactive scheme.

When the core activity level is greater than $50 \%$, the reactive algorithm selects a higher frequency to increase performance enough to allow the core to be idle more than $50 \%$ (i.e. active $<50 \%$ ) of the time. The new frequency is selected assuming a $100 \%$ frequency increase reduces active time by $50 \%$. For example assume a core operates at $1 \mathrm{GHz}$ and is $100 \%$ active. In order to achieve an activity level of $50 \%$, the algorithm would attempt to double the frequency to $2 \mathrm{GHz}$. Frequency reductions are similar in that activity levels below $30 \%$ cause the algorithm to reduce frequency in order to increase 
activity levels to $30 \%$. Since processors have a finite number of architected DVFS states, the algorithm selects the nearest frequency which meets the target activity level.

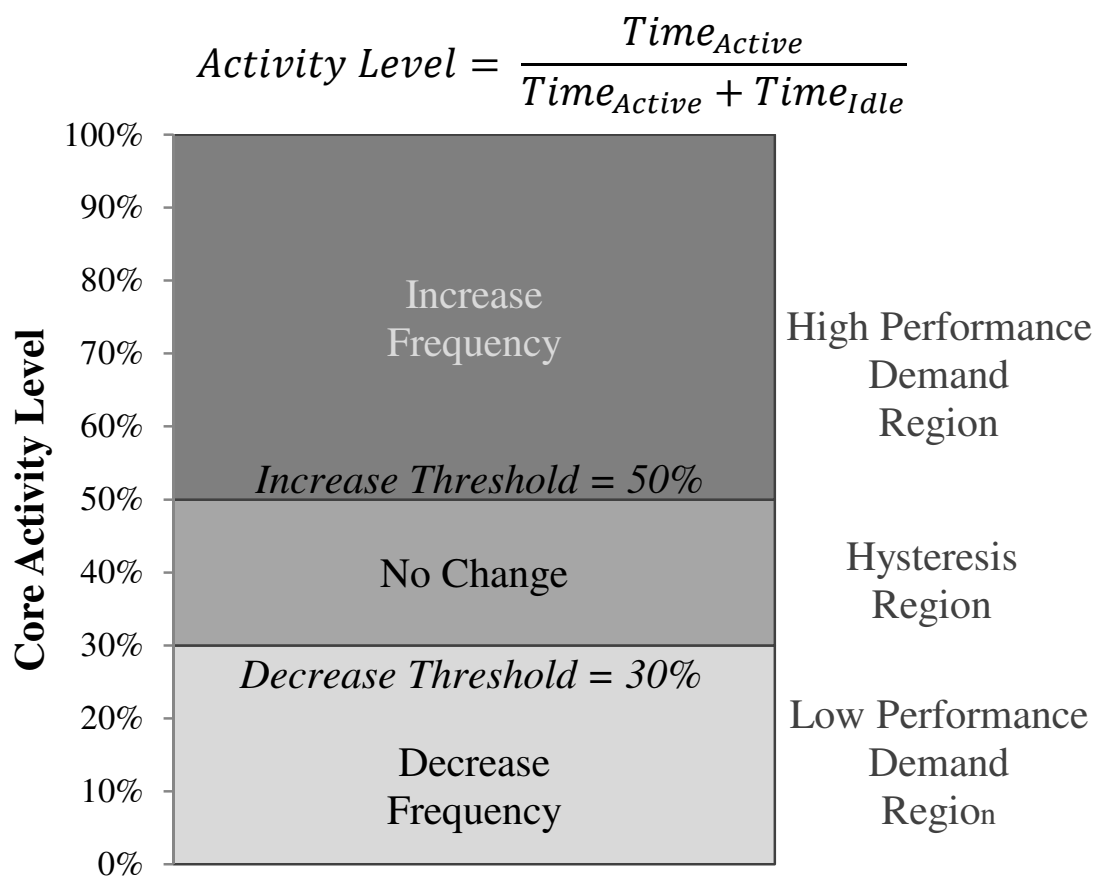

Figure 7.2: Windows Vista Reactive P-State Selection Algorithm

\subsection{Workload Characterization}

To analyze the power/performance impact of the predictive power management scheme on real-world workloads, a system running the desktop/client SYSmark 2007 benchmark is characterized. This benchmark represents a wide range of desktop computing applications. The benchmark components are E-Learning, Video Creation, Productivity, and 3D. The individual subtests are listed in Table 7.1. This benchmark is particularly important to the study of dynamic power adaptations since it provides realistic user scenarios that include user interface and I/O delays. These delays cause a large amount 
of idle-active transitions in the cores. Since current OSs determine dynamic adaption levels using core activity level, the replication of these user interactions in the benchmark is critical. For comparison, Figure 7.3 shows average active core residencies of a sixcore AMD Phenom 2 processor across a wide range of desktop workloads. The left-hand side of the figure illustrates the problem with many popular benchmark applications. The benchmarks spend nearly $100 \%$ of the time with all cores active. From an idle power management perspective little can be done to improve performance and power efficiency. The center and right-hand side workloads are more challenging for idle power management due to the frequent occurrence of idle phases. The SYSmark subtests are the most challenging due to their large composition of interspersed idle and active phases.

Table 7.1: SYSmark 2007 Components

\begin{tabular}{|c|c|c|c|}
\hline E-Learning & Video Creation & Productivity & $3 \mathrm{D}$ \\
\hline $\begin{array}{l}\text { Adobe } \\
\text { - Illustrator } \\
\text { - Photoshop } \\
\text {-Flash } \\
\text { Microsoft } \\
\text { - PowerPoint }\end{array}$ & $\begin{array}{l}\text { Adobe } \\
\text { - After Effects } \\
\text { - Illustrator } \\
\text { - Photoshop } \\
\text { Microsoft } \\
\text { - Media Encoder } \\
\text { Sony } \\
\text { - Vegas }\end{array}$ & $\begin{array}{l}\text { Microsoft } \\
\text { - Excel } \\
\text { - Outlook } \\
\text { - Word } \\
\text { - PowerPoint } \\
\text { - Project } \\
\text { Corel } \\
\text { - WinZip }\end{array}$ & $\begin{array}{l}\text { Autodesk } \\
\text { - 3Ds Max } \\
\text { Google } \\
\text { - SketchUp }\end{array}$ \\
\hline \multicolumn{4}{|c|}{ Performance Loss for Vista Reactive Power Management [4] } \\
\hline $8.8 \%$ & $6.2 \%$ & $9.5 \%$ & $5.9 \%$ \\
\hline
\end{tabular}




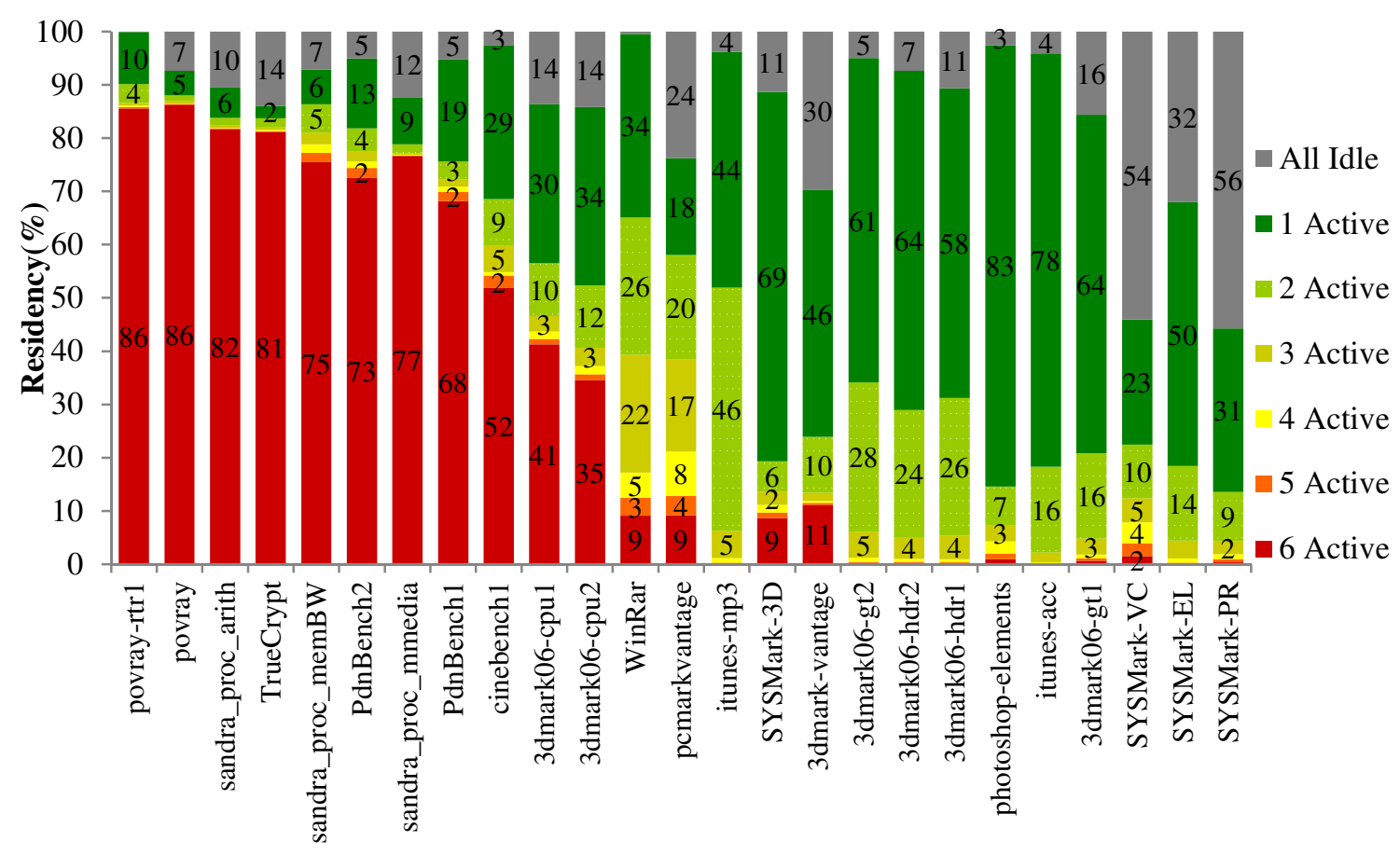

Figure 7.3: Six-Core Phenom 2 Desktop Activity Levels

Figure 7.4 takes the active-idle analysis of SYSmark to a deeper level by showing the core activity level within each subtest. Unlike traditional scientific and computing benchmarks, core activity level varies greatly over time. The primarily single-thread ELearning and Productivity subtests are composed of single core active phases interspersed with all cores idle. The frequent transitions between active and idle make power management decisions difficult. Reacting too quickly to idle phases can induce excessive performance loss as code execution is halted to allow transition of clocks, voltage planes or component state. At the other extreme the 3D and Video Creation workloads have large sections of all cores being active. These regions, also interspersed with all-idle and one-active regions are critical for power sharing strategies. As the 
workload/operating systems adds and removes threads from cores the resultant power level changes drastically. The power difference between cores in the active versus idle state is much greater than differences within the active state.
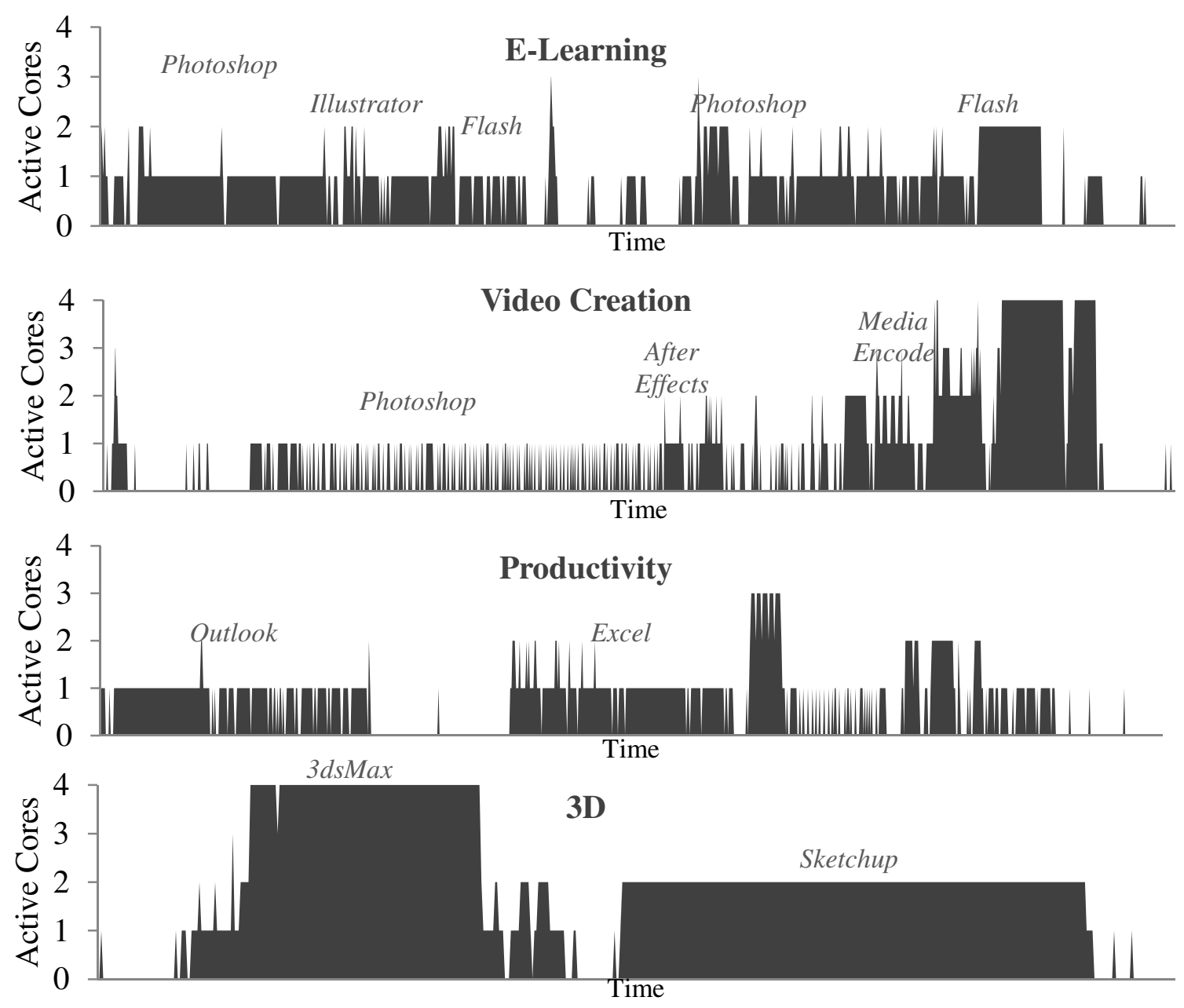

Productivity

Figure 7.4: Utilization of Multiple Cores by SYSmark 2007 Benchmark

The effect of these frequent transitions on power and performance is significant. Bircher et al [BiJo08] show that the slow reaction of the Vista DVFS algorithm leads to a performance loss of 6\%-10\% for SYSmark 2007. It is shown that this performance loss is due to a large portion of the benchmark operating at DVFS state with frequencies as 
low as $1 / 3$ of the maximum frequency. Similarly, power consumption is excessively high due to the DVFS algorithm choosing high-power states for many of the frequent, idle phases in the benchmark.

\subsection{Periodic Power Phase Predictor - PPPP}

While power management in operating systems like Windows/Linux is reactive, there have been proposals to use predictive power management [IsBu06] [DuCa03] [DiSo08]. Isci [IsBu06] uses table-based predictors of memory operations/instruction, to direct DVFS decisions for single-threaded workloads. Duesterwald et al. [DuCa03] examine table-based predictor techniques to predict performance-related metrics (IPC, cache misses/instruction and branch misprediction rates) of single-thread workloads, but not power. Diao [DiSo08] uses machine learning to predict activity patterns. The predictions are used to make policy decisions for entering core idle states. In contrast, the periodic power phase predictor (PPPP) is proposed which makes use of table-based prediction structures and the repetitive nature of power phases to predict performance demand and/or power consumption. The predictor is shown in Figure 7.5. Like traditional table-based predictors, the main components are: a global phase history register (GPHR), pattern history table (PHT) and predicted level. Typically, table-based predictors track sequences of events such as branch outcomes or IPC samples [DuCa03] [IsBu06]. This predictor is distinct in that it tracks run-length-encoded sequences of core active/idle phases. Activity in this case is defined as execution of architected 
instructions. In APCI[Ac07] terminology that is popularly used for power management, it is known as the $\mathrm{C}_{0}$ state. All other time is considered non-active or idle. Idle time is explicitly defined as "executing" in a processor idle state via the HLT instruction or other idle state entry method [Bk09]. This state is also known as the $\mathrm{C}_{\mathrm{x}}$ state where $\mathrm{x}=1$ to $\mathrm{N}$. These non- $\mathrm{C}_{0}$ states are responsible for the largest power reductions due to the application of clock and power gating. Large power changes or phases can be detected by tracking core activity patterns. For this reason the PPPP is constructed to track core activity patterns.

A diagram of the predictor is provided in Figure 7.5. The main feature of the predictor is its ability to capture frequent, power-relevant events by tracking active/idle patterns. Transitions to active or idle states and the resultant power level can be predicted by tracking previous patterns. One of the most common events is the periodic timer tick event used in many commercial operating systems [SiPa07]. This event occurs on a regular interval to provide timing and responsiveness to the operating system scheduler. When a core is otherwise idle, the timer tick produces an easily discernable pattern. 


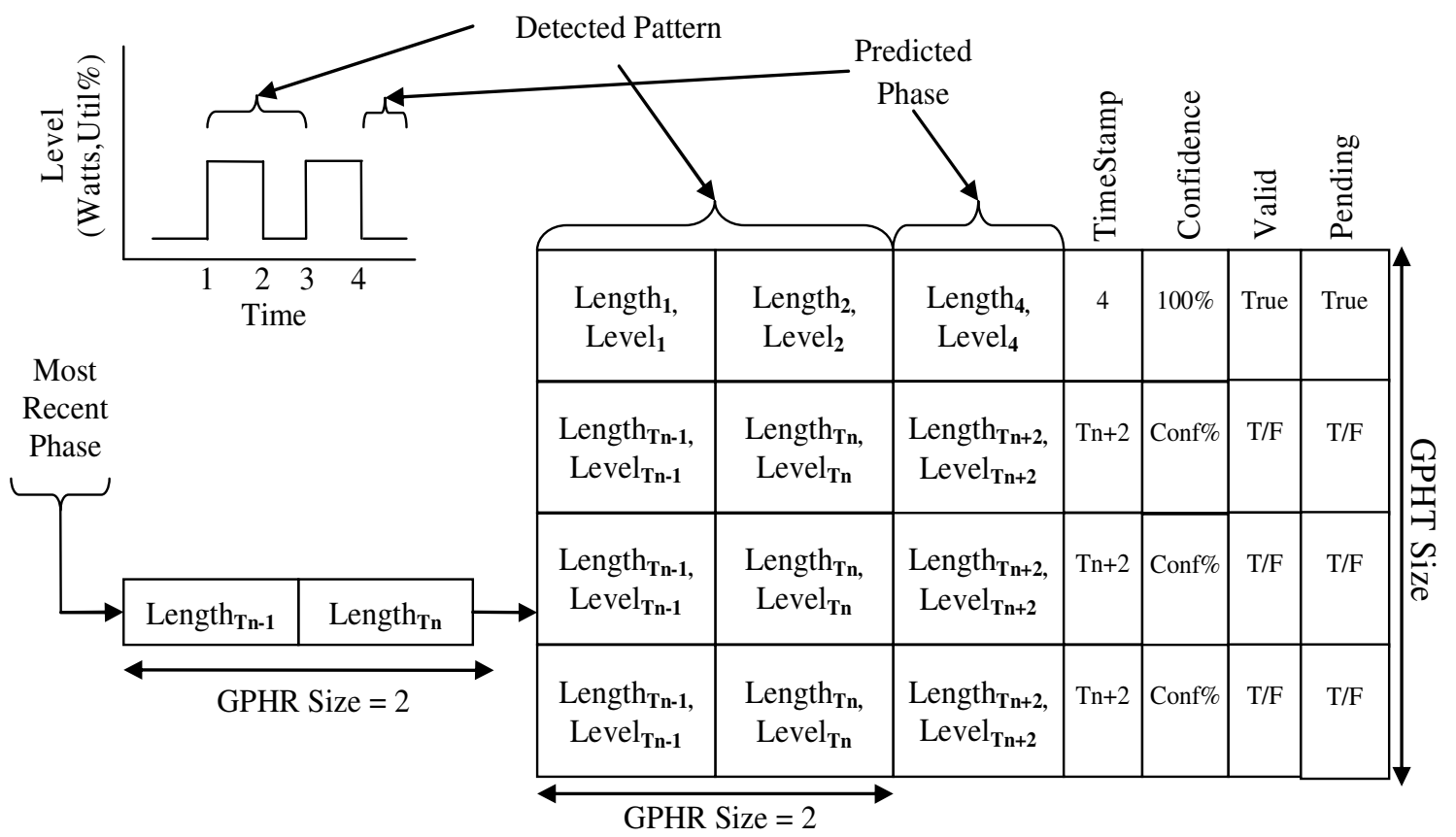

Figure 7.5: Periodic Power Phase Predictor

For Windows operating systems, the boot strap processor remains idle for periods of 16 milliseconds interrupted by active periods lasting about 100 microseconds.

The predictor tracks the duration of the idle and active phases in the length and level fields. As a pattern of active and idle period repeats the predictor updates the quality of the prediction using the confidence field. When a pattern is first identified, it is copied to the GPHT and assigned a confidence level of $100 \%$. The correctness of the prediction is assessed by comparing the predicted time and level of the next transition (timestamp field) to the actual transition. If a misprediction in duration or level is found, the confidence is reduced by a tunable percentage. If multiple mispredictions occur, the 
confidence level will drop below a confidence limit. The net effect of these parameters is that a prediction will be invalidated after three consecutive mispredictions. The valid and pending fields are used to determine which predictor entries can be used for predictor matches and which have outstanding predictions respectively. Multiple predictions can be outstanding. If conflicting prediction exist at an evaluation point, the higher confidence prediction is used. For equal confidence predictions, the lower index prediction is used. Additional details for each predictor field are provided in Table 7.2. An example of the PPPP tracking and predicting core utilization level is given in Figure 7.6 .
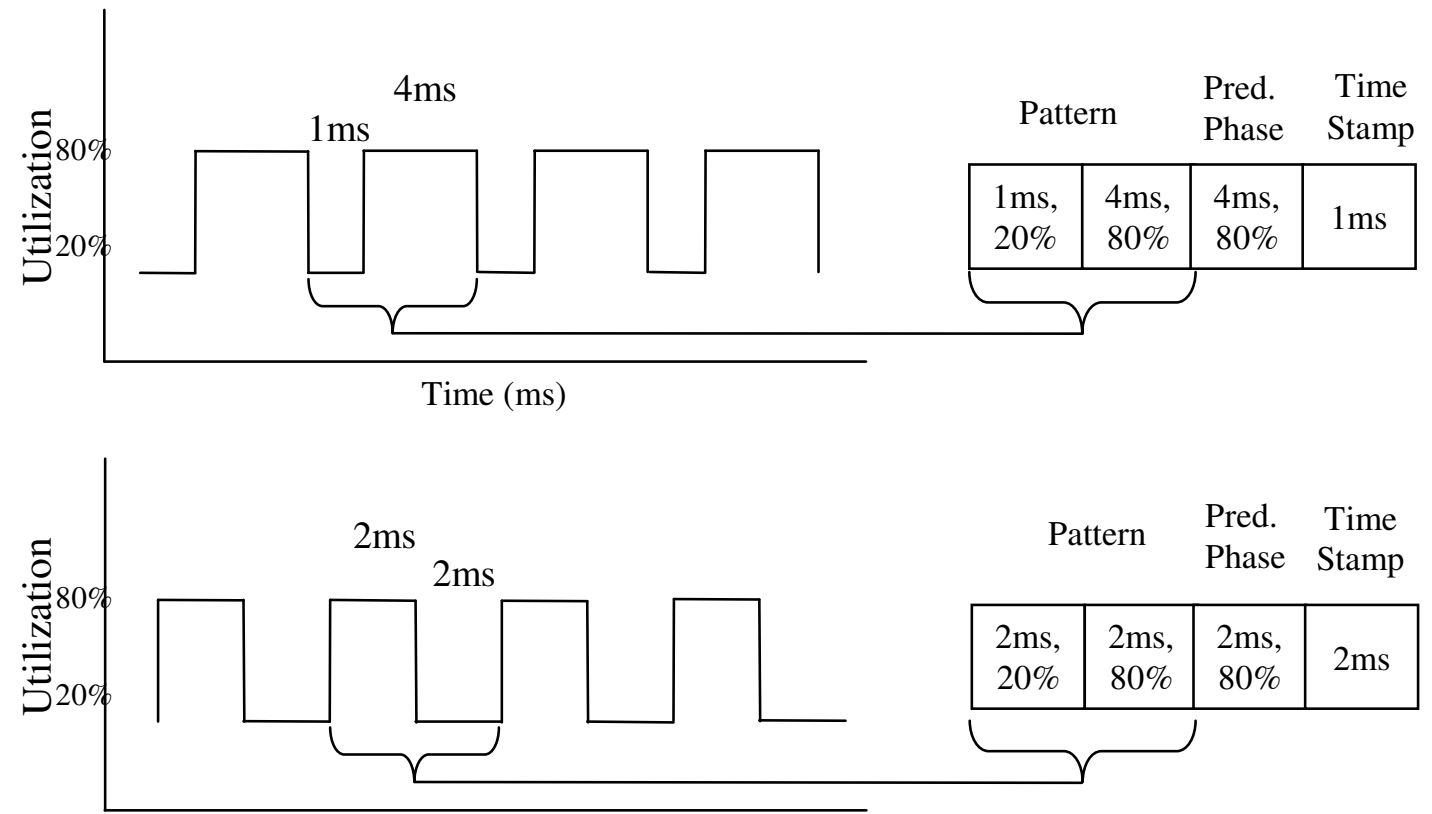

Time (ms)

Figure 7.6: Example of Program Phase Mapping to Predictor 


\section{Table 7.2: Periodic Power Phase Predictor Field Descriptions}

\begin{tabular}{|c|c|}
\hline $\begin{array}{l}\text { Predictor } \\
\text { Field }\end{array}$ & Description \\
\hline $\begin{array}{l}\text { Detected } \\
\text { Pattern } \\
\text { Length,Level }\end{array}$ & $\begin{array}{l}\text { Duration and level of phase pair. This is also the table index. When a } \\
\text { periodic phase is detected, it is used to index the prediction table. }\end{array}$ \\
\hline $\begin{array}{l}\text { Predicted } \\
\text { Phase } \\
\text { Length,Level }\end{array}$ & $\begin{array}{l}\text { Predicted Phase at next transition. For utilization predictor this is activity } \\
\text { level. For power prediction this is the power level seen when this phase } \\
\text { previously occurred. }\end{array}$ \\
\hline Timestamp & $\begin{array}{l}\text { Records timestamp of when predicted phase change is to occur. This is } \\
\text { the most critical value produced by the predictor. It is used by the power } \\
\text { manager to schedule changes in power/performance capacity of the } \\
\text { system. This value allows for optimal selection of performance capacity } \\
\text { given the anticipated duration of operation at a particular performance } \\
\text { demand. }\end{array}$ \\
\hline Confidence & $\begin{array}{l}\text { "Quality" of phase as a function of past predictions and duration. The } \\
\text { confidence is used by the power manager to determine if a prediction will } \\
\text { be used or not. It is also used by the replacement algorithm to determine } \\
\text { if the phase will be replaced if the predictor is full. All newly detected } \\
\text { phases start with a confidence of } 1 \text {. If the phase is subsequently } \\
\text { mispredicted, the confidence is reduced by a fixed ratio. }\end{array}$ \\
\hline Valid & Indicates if this entry has a valid phase stored with a "true" or "false." \\
\hline Pending & $\begin{array}{l}\text { Indicates if this phase is predicted to occur again. This value is set "true" } \\
\text { on the occurrence of the phase and remains true until the phase prediction } \\
\text { expires. }\end{array}$ \\
\hline
\end{tabular}

\subsection{Predicting Core Activity Level}

This section provides power and performance results for the core and aggregate-level periodic power phase predictor in comparison to a commercial reactive scheme. A comparison is made in terms of prediction accuracy, prediction coverage, power and performance. Also, a characterization of core activity phases is given. 
First, prediction accuracy is considered. Accuracy is defined according to the, commercial, reactive DVFS algorithm used in the Windows Vista operating system [Vi07]. A correct prediction is one in which the selected DVFS frequency selection keeps the processor within the target range of $30 \%$ to $50 \%$ activity.

The accuracy of the reactive scheme is determined by analyzing traces of core DVFS and activity levels from a real system. If the selected frequency did not cause the core to have an activity level between $30 \%$ and $50 \%$, the selection is considered wrong. For the predictive schemes, the activity level trace is played back through the predictor while allowing it to select a frequency to meet the $30 \%-50 \%$ target. Since core activity level changes according to core frequency, the resultant activity level must be scaled accordingly. The amount of scaling is determined experimentally by measuring performance of the SYSmark workload under a range of core frequencies. Performance, and therefore activity level, scale $70 \%$ for each $100 \%$ change in core frequency.

Using this approach results are presented for SYSmark 2007 prediction accuracy in Table 7.3. DVFS hit rate is provided for three predictors. Core-level PPPP represents the predictor applied to each core. Aggregate PPPP represents the predictor driven by the total activity level. All target activity levels remain the same. A single predictor, driven by the aggregate activity level (i.e. average of all cores) is used to select the next core frequency. Core-level reactive represents the Windows Vista DVFS algorithm. 
Table 7.3: SYSmark 2007 DVFS Hit Rate

\begin{tabular}{|c|c|c|c|c|}
\hline Predictor & E-Learning & Productivity & Video Creation & $3 \mathrm{D}$ \\
\hline $\begin{array}{c}\text { Core-Level } \\
\text { PPPP }\end{array}$ & $82.6 \%$ & $73.8 \%$ & $76.4 \%$ & $72.7 \%$ \\
\hline $\begin{array}{c}\text { Aggregate } \\
\text { PPPP }\end{array}$ & $26.8 \%$ & $26.3 \%$ & $40.2 \%$ & $30.7 \%$ \\
\hline Core-Level Reactive (Vista) & $66.4 \%$ & $65.2 \%$ & $63.5 \%$ & $59.7 \%$ \\
\hline
\end{tabular}

The limitations of reactive DVFS selection are evident. Due to frequent transitions between high and low activity levels, the reactive scheme is only able to achieve the correct frequency about $2 / 3$ of the time. PPPP applied at the aggregate level is much worse with an average of $31 \%$ accuracy. The best case is achieved with the core-level PPPP which averages $76 \%$. The differences in the success of these predictors are a result of prediction coverage and accuracy of the predicted phases. See Table 7.4. Coverage is defined as percentage of the workload in which a prediction is available. A prediction could be unavailable if the last observed activity pattern has not been seen before or has caused too many mispredictions. The reactive scheme does not have coverage since it does not predict. In contrast PPPP has much lower prediction coverage, especially for the aggregate predictor. The aliasing of multiple core phases obscures predictable behavior to less than 3\% for E-Learning and Productivity. Video Creation and 3D are slightly better at $16 \%$ and $8 \%$ respectively. One possible reason is that these workloads have larger portions of multi-threaded execution. The aggregate activity level is likely more representative of core-level activity compared to the single-threaded E-Learning and Productivity. Core-level PPPP achieves the highest accuracy by having a large workload 
coverage of $43 \%$ and accuracy over $95 \%$ in the covered portion. Outside of the covered portions the predictor selects frequency according to the reactive algorithm.

Table 7.4: SYSmark 2007 Prediction Coverage

\begin{tabular}{|c|c|c|c|c|}
\hline Predictor & E-Learning & Productivity & Video Creation & $3 \mathrm{D}$ \\
\hline $\begin{array}{c}\text { Core-Level } \\
\text { PPPP }\end{array}$ & $57.0 \%$ & $33.5 \%$ & $43.0 \%$ & $37.9 \%$ \\
\hline $\begin{array}{c}\text { Aggregate } \\
\text { PPPP }\end{array}$ & $1.3 \%$ & $2.3 \%$ & $16.3 \%$ & $8.0 \%$ \\
\hline Core-Level Reactive (Vista) & N/A & N/A & N/A & N/A \\
\hline
\end{tabular}

To quantify the improved predictability of core-level versus aggregate PPPP, Table 7.5 presents a characterization of core active and idle durations for SYSmark 2007. Durations group into the following ranges: $<10$ milliseconds, 10-100 milliseconds, 1001000 milliseconds and > 1000 milliseconds. One of the major distinctions between corelevel and Aggregate is the high concentration of short phases, less than $10 \mathrm{~ms}$ for CoreTotal. Just as in the example shown in Figure 1.4, these short phases are largely a result of misalignment of the core-level activity. In particular, the most common phases are in the $10-100 \mathrm{~ms}$ range. This is caused by the timer tick, scheduling and power adaptation intervals for the Windows operating systems. The timer tick normally occurs on $16 \mathrm{~ms}$ boundaries. Thread creation and migration events also occur on these boundaries. Power adaptations (DVFS) occur on 100ms boundaries. Therefore, idle phases are frequently interrupted by these events. Similarly, active phases are often terminated by threads being migrated to other cores on these same boundaries. Any misalignment of these events between cores causes the effective activity durations to be 
shorter and less predictable. Further evidence of these common 10-100ms phases is given in Figure 7.7 which shows the frequency distribution of active and idle phases across SYSmark 2007.

Table 7.5: Core Phase Residency by Length

\begin{tabular}{|c|c|c|c|c|}
\hline & \multicolumn{2}{|c|}{ E-Learning } & \multicolumn{2}{c|}{ Video Creation } \\
\hline Phase Length & Core & Aggregate & Core & Aggregate \\
\hline Less Than $10 \mathrm{~ms}$ & $11 \%$ & $93 \%$ & $44 \%$ & $82 \%$ \\
\hline $10-100 \mathrm{~ms}$ & $49 \%$ & $7 \%$ & $27 \%$ & $2 \%$ \\
\hline $100-1000 \mathrm{~ms}$ & $10 \%$ & $0 \%$ & $14 \%$ & $9 \%$ \\
\hline Greater Than $1000 \mathrm{~ms}$ & $30 \%$ & $0 \%$ & $16 \%$ & $7 \%$ \\
\hline & \multicolumn{2}{|c|}{ Productivity } & \multicolumn{2}{|c|}{$3 \mathrm{D}$} \\
\hline Phase Length & Core & Aggregate & Core & Aggregate \\
\hline Less Than $10 \mathrm{~ms}$ & $55 \%$ & $97 \%$ & $55 \%$ & $97 \%$ \\
\hline $10-100 \mathrm{~ms}$ & $30 \%$ & $3 \%$ & $30 \%$ & $3 \%$ \\
\hline $100-1000 \mathrm{~ms}$ & $5 \%$ & $0 \%$ & $5 \%$ & $0 \%$ \\
\hline Greater Than $1000 \mathrm{~ms}$ & $11 \%$ & $0 \%$ & $11 \%$ & $0 \%$ \\
\hline
\end{tabular}

Active and idle phase as considered as a group since both are relevant for prediction. Idle phases must be predicted in order to anticipate how long a power surplus will be available. Similarly, active phase must be predicted to anticipate durations of power deficits. In both cases the predicted durations is needed in order to weigh the power and performance cost of transitioning to low power states or changing the DVFS operating point. Several local maximums are present due to the periodic nature of the interaction between power management, OSs and system hardware. By removing or varying the intensity of these various events and observing the change in frequency distribution, the period length may be related to its source. Note the prevalence of phases in the $10-15 \mathrm{~ms}$ range that corresponds to the OS scheduling interval. Also, consider the spikes at $100 \mathrm{~ms}$, 
which corresponds to the DVFS scheduling interval. Additional, longer-duration maximums occur in the $200 \mathrm{~ms}$ and higher range. These correspond to GUI interaction and I/O delays occurring in the SYSmark benchmark.

Next the resultant power and performance impact of the core-level PPPP versus reactive DVFS selection is considered. Aggregate PPPP is not considered due its poor prediction accuracy. Table 7.6 presents power and performance results for the two schemes. Power and performance are estimated using the measured and predicted DFVS, active and idle states shown in Table 7.7. On average, power is reduced by $5.4 \%$ while achieving a speedup of $3.8 \%$. This improvement is caused by PPPP more frequently selecting high frequencies for active phases and low frequencies for performance-insensitive idle phases. This shift can be seen in the active residencies of all subtests. The $2.4 \mathrm{GHz}-$ Active state increases by 0.6 to 2.5 percentage points. Similarly, the active time in lower frequencies is reduced an average of 0.76 percentage points. The performance impact of selecting a low frequency for an active phase can be large. For example, selecting $800 \mathrm{MHz}$ rather than $2.4 \mathrm{GHz}$ yields a performance loss of $47 \%((1-0.8 \mathrm{GHz} / 2.4 \mathrm{GHz}) \mathrm{x}$ 70\%). Therefore, it takes only a small change in residency to drastically impact performance. Also, the impact on performance is larger due to active time representing only an average of $17 \%$ total time. This magnifies the performance impact by about $6 \mathrm{x}$ (1/0.17). The net effect on active frequency is an increase of $144 \mathrm{MHz}$ from $1.55 \mathrm{GHz}$ to 1.69GHz. Note that though frequency increases by $9.3 \%$, performance increases only 
$3.8 \%$ due to limited frequency scaling of the workload $(70 \%)$ and reduced total time in the active state.

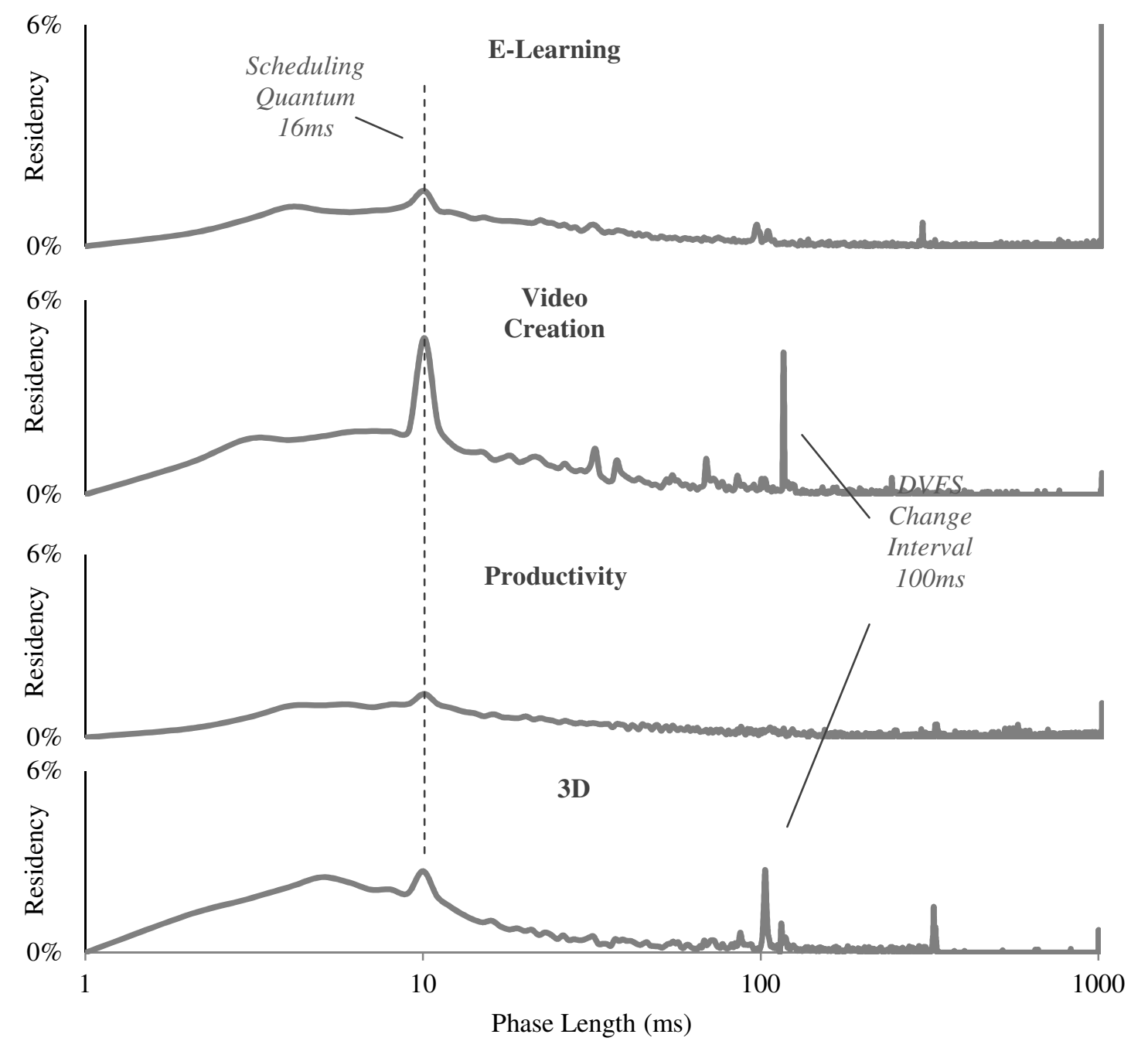

Figure 7.7 Core-Level Phase Length Probability Distributions

Next, power savings is considered. Though it is possible to bias a reactive DVFS algorithm to achieve performance comparable to a predictive algorithm, it is not possible to do so without increasing power consumption drastically. Prediction allows DVFS selection to select the "correct" frequency for both performance and power savings. 
Table 7.6: SYSmark 2007 Power and Performance Impact of PPPP

\begin{tabular}{|c|c|c|c|c|c|}
\hline & \multicolumn{2}{|c|}{ E-Learning } & \multicolumn{2}{c|}{ Productivity } \\
\hline & $\begin{array}{c}\text { Predictive } \\
\text { (PPPP) }\end{array}$ & $\begin{array}{c}\text { Reactive } \\
\text { (Vista) }\end{array}$ & $\begin{array}{c}\text { Predictive } \\
\text { (PPPP) }\end{array}$ & $\begin{array}{c}\text { Reactive } \\
\text { (Vista) }\end{array}$ \\
\hline Power (W) & 16.6 & 18.2 & 14.3 & 15.1 \\
\hline Power Savings & \multicolumn{2}{|c|}{$8.3 \%$} & \multicolumn{2}{c|}{$5.3 \%$} \\
\hline Delay (sec) & 924 & 963 & 585 & 607 \\
\hline Speedup & \multicolumn{2}{|c|}{$4.2 \%$} & \multicolumn{2}{|c|}{$3.7 \%$} \\
\hline Energy (KJ) & 15.4 & 17.5 & 8.4 & 9.2 \\
\hline Energy x Delay(KJs) & $14.2 \times 10^{3}$ & $16.9 \times 10^{3}$ & $4.9 \times 10^{3}$ & $5.6 \times 10^{3}$ \\
\hline Energy x Delay(KJs ${ }^{2}$ ) & $13.2 \times 10^{6}$ & $16.2 \times 10^{6}$ & $2.9 \times 10^{6}$ & $3.4 \times 10^{6}$ \\
\hline Energy Savings & \multicolumn{2}{|c|}{$12.3 \%$} & \multicolumn{3}{|c|}{$8.7 \%$} \\
\hline
\end{tabular}

\begin{tabular}{|c|c|c|c|c|c|}
\hline & \multicolumn{2}{|c|}{ Video Creation } & \multicolumn{2}{c|}{ 3D } \\
\hline & $\begin{array}{c}\text { Predictive } \\
\text { (PPPP) }\end{array}$ & $\begin{array}{c}\text { Reactive } \\
\text { (Vista) }\end{array}$ & $\begin{array}{c}\text { Predictive } \\
\text { (PPPP) }\end{array}$ & $\begin{array}{c}\text { Reactive } \\
\text { (Vista) }\end{array}$ \\
\hline Power (W) & 18.6 & 19.5 & 25.9 & 26.6 \\
\hline Power Savings & \multicolumn{2}{|c|}{$4.7 \%$} & \multicolumn{2}{c|}{$2.9 \%$} \\
\hline Delay (sec) & 1129 & 1172 & 548 & 568 \\
\hline Speedup & \multicolumn{2}{|c|}{$3.8 \%$} & \multicolumn{2}{|c|}{$3.6 \%$} \\
\hline Energy (KJ) & 20.9 & 22.8 & 14.2 & 15.1 \\
\hline Energy x Delay(KJs) & $23.6 \times 10^{3}$ & $26.7 \times 10^{3}$ & $7.8 \times 10^{3}$ & $8.6 \times 10^{3}$ \\
\hline Energy x Delay(KJs ${ }^{2}$ ) & $26.7 \times 10^{6}$ & $31.3 \times 10^{6}$ & $4.3 \times 10^{6}$ & $4.9 \times 10^{6}$ \\
\hline Energy Savings & \multicolumn{2}{|c|}{$8.2 \%$} & \multicolumn{2}{|c|}{$6.3 \%$} \\
\hline
\end{tabular}


Table 7.7: SYSmark 2007 P-State and C-State Residency of PPPP versus Reactive

\begin{tabular}{|c|c|c|c|c|}
\hline & \multicolumn{2}{|c|}{ E-Learning } & \multicolumn{2}{|c|}{ Productivity } \\
\hline & $\begin{array}{c}\text { Predictive } \\
\text { (PPPP) }\end{array}$ & $\begin{array}{l}\text { Reactive } \\
\text { (Vista) }\end{array}$ & $\begin{array}{l}\text { Predictive } \\
\text { (PPPP) }\end{array}$ & $\begin{array}{c}\text { Reactive } \\
\text { (Vista) }\end{array}$ \\
\hline 2.4GHz - Active & $5.4 \%$ & $4.6 \%$ & $2.9 \%$ & $2.4 \%$ \\
\hline 2.4GHz - Idle & $7.1 \%$ & $17.4 \%$ & $4.4 \%$ & $9.6 \%$ \\
\hline 1.6GHz - Active & $1.2 \%$ & $1.4 \%$ & $0.8 \%$ & $0.8 \%$ \\
\hline 1.6GHz - Idle & $5.5 \%$ & $9.4 \%$ & $3.8 \%$ & $6.2 \%$ \\
\hline 1.2GHz - Active & $1.1 \%$ & $1.2 \%$ & $1.2 \%$ & $1.2 \%$ \\
\hline $1.2 \mathrm{GHz}-$ Idle & $6.9 \%$ & $9.8 \%$ & $6.6 \%$ & $9.7 \%$ \\
\hline $0.8 \mathrm{GHz}$ - Active & $3.2 \%$ & $4.5 \%$ & $3.8 \%$ & $4.7 \%$ \\
\hline $0.8 \mathrm{GHz}-$ Idle & $69.5 \%$ & $51.8 \%$ & $76.5 \%$ & $65.3 \%$ \\
\hline Active Frequency & $1.72 \mathrm{GHz}$ & $1.56 \mathrm{GHz}$ & $1.47 \mathrm{GHz}$ & $1.34 \mathrm{GHz}$ \\
\hline \multirow[t]{3}{*}{$\begin{array}{c}\text { Idle } \\
\text { Frequency }\end{array}$} & $1.01 \mathrm{GHz}$ & $1.24 \mathrm{GHz}$ & $0.94 \mathrm{GHz}$ & $1.07 \mathrm{GHz}$ \\
\hline & \multicolumn{2}{|c|}{ Video Creation } & \multicolumn{2}{|c|}{$3 \mathrm{D}$} \\
\hline & $\begin{array}{c}\text { Predictive } \\
\text { (PPPP) }\end{array}$ & $\begin{array}{l}\text { Reactive } \\
\text { (Vista) }\end{array}$ & $\begin{array}{l}\text { Predictive } \\
\text { (PPPP) }\end{array}$ & $\begin{array}{c}\text { Reactive } \\
\text { (Vista) }\end{array}$ \\
\hline 2.4GHz - Active & $6.8 \%$ & $5.3 \%$ & $17.5 \%$ & $15.0 \%$ \\
\hline $2.4 \mathrm{GHz}$ - Idle & $5.7 \%$ & $12.4 \%$ & $7.8 \%$ & $17.0 \%$ \\
\hline 1.6GHz - Active & $2.7 \%$ & $3.2 \%$ & $3.9 \%$ & $5.1 \%$ \\
\hline $1.6 \mathrm{GHz}$ - Idle & $5.6 \%$ & $9.6 \%$ & $4.6 \%$ & $6.7 \%$ \\
\hline 1.2GHz - Active & $3.8 \%$ & $4.8 \%$ & $2.6 \%$ & $3.1 \%$ \\
\hline $1.2 \mathrm{GHz}$ - Idle & $9.2 \%$ & $13.8 \%$ & $7.3 \%$ & $9.4 \%$ \\
\hline $0.8 \mathrm{GHz}$ - Active & $3.7 \%$ & $4.8 \%$ & $4.7 \%$ & $6.9 \%$ \\
\hline $0.8 \mathrm{GHz}$ - Idle & $62.3 \%$ & $46.1 \%$ & $51.6 \%$ & $36.7 \%$ \\
\hline Active Frequency & $1.65 \mathrm{GHz}$ & $1.51 \mathrm{GHz}$ & $1.92 \mathrm{GHz}$ & $1.77 \mathrm{GHz}$ \\
\hline $\begin{array}{c}\text { Idle } \\
\text { Frequency }\end{array}$ & $1.01 \mathrm{GHz}$ & $1.20 \mathrm{GHz}$ & $1.07 \mathrm{GHz}$ & $1.32 \mathrm{GHz}$ \\
\hline
\end{tabular}


In this case the predictor achieves a $3.8 \%$ performance increase while reducing power consumption by $5.4 \%$. The primary cause is a shift in idle frequency selections away from the high-performance, high-leakage states. Residency in the most inefficient state, $2.4 \mathrm{GHz}$ - Idle, is reduced by an average of 7.8 percentage points. Residency in other idle states above the minimum frequency also decreased, but by a smaller 3.1 percentage points. This increases idle residency in the minimum frequency idle state of $800 \mathrm{MHz}$ by an average of $15 \%$. Average idle frequency decreases by $200 \mathrm{MHz}$ from $1.2 \mathrm{GHz}$ to $1.0 \mathrm{GHz}$.

\subsection{Predicting Power Levels}

The second application of periodic power phase prediction is for predicting core power consumption. Predicting power levels provides opportunities for increased performance and efficiency. Existing power control systems such as power capping[Po10] and turbo boost [ChJa09] apply power and performance limits statically based on user-specified or instantaneous power consumption. Knowing power levels a priori could increase performance by avoiding adaptations for short duration phases. For example, a core that encounters a short, high-power phase of execution may cause the power controller to reduce its or other processors' frequency. If the controller could know that the phase would be too short to cause a power or temperature violation, the reduction in performance could be avoided. 
To this end PPPP is applied to predict the core-level and aggregate power consumption. Results are compared to a last value predictor also at the core and aggregate level. Corelevel power is measured using the PMC-based power model. The model allows finegrain, power management and temperature-aware estimation of core power.

Rather than using core activity level to predict core activity level, it is used to cross predict power level. The predicted activity-level in the predictor is replaced by the modeled core power level. The prediction table index remains as sequences of core activity levels. This approach provides better pattern matching as variations in temperature and application of DVFS tends to hide otherwise discernable patterns.

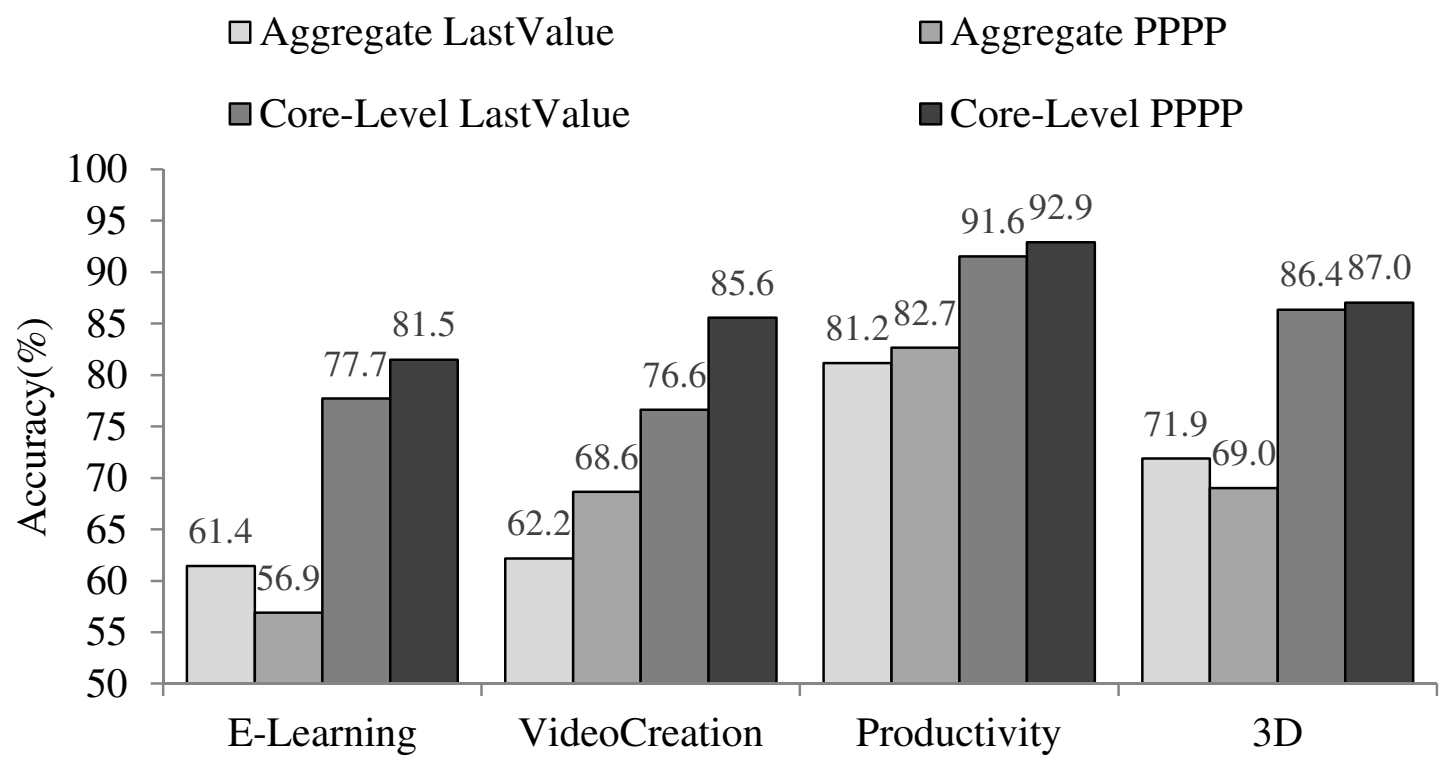

Figure 7.8 Prediction Accuracy of Core Power for Various Predictors

Figure 7.8 shows the weighted average percent accuracy of the periodic power phase predictor compared to a last-value predictor. Weighted average is chosen since SYSmark 
2007 power consumption contains many idle, low-power phases. In these phases, a small error in absolute terms yields a large percentage error. Therefore, error values are scaled by the magnitude of measured power sample compared to the maximum observed. For example, a $10 \%$ error on a $5 \mathrm{~W}$ sample has half the impact of a $10 \%$ error on a $10 \mathrm{~W}$ sample. For all subtests, the core-level versions of the predictors outperformed the aggregate versions. The best overall performance is $86 \%$ accuracy for the periodic corelevel predictor compared to $83 \%$ for the core-level version of the last-value predictor. The benefit of core-level prediction of power is less pronounced than for prediction of activity level. This is due to the smaller dynamic range of power consumption compared to activity level. Though activity levels regularly vary from $0 \%$ to $100 \%$, power levels remain in a much smaller range of approximately $25 \%$ to $75 \%$.

\subsection{Summary}

This section presents the concept of core-level phase prediction and its application to dynamic power management. By observing changes in performance demand and power consumption at the core-level, it is possible to perceive predictable phase behavior. Prediction of phases allows power management to avoid over or under provisioning resources in response to workload changes. Using this concept the PPPP is developed. It is a simple, table-based prediction scheme for directing DVFS selection. It is applied to the SYSmark2007 benchmark suite and attain significant performance and power improvements. Compared to the reactive DVFS algorithm used by Windows Vista, 
performance is increased by $5.4 \%$ and while power consumption is reduced by $3.8 \%$. We show that processor power can be predicted by PPPP with accuracy $4.8 \%$ better than a last-value predictor.

Predictive schemes such as PPPP are the next step in improving performance and efficiency of systems employing dynamic power management. As it was demonstrated in the pursuit of higher single-threaded processor performance, the highest possible performance is achieved when costly phase changes can be predicted. Prediction allows the use of more aggressive power saving techniques since excessive performance loss can be avoided. 


\section{Chapter 8 Related Research}

This section summarizes related research in the areas relating to predictive processor power management. Specifically, performance counter-driven power models, systemlevel power characterization and predictive power management are covered.

\subsection{Performance Counter-Driven Power Models}

Contemporary research in the area of performance counter-driven power modeling has focused primarily on the single largest consumer, the processor [LiJo03] [Be00] [IsMa03] [CoMa05] [BrTiMa00]. System-level power modeling [HeCe06] is mostly focused on power consumption within a single subsystem [Ja01] [ZeSo03] [KiSu06] [GuSi02].

Consistent throughout processor power modeling is the theme that power consumption is primarily determined by the number of instructions retired per cycle. Li et al [LiJo03] present a simple linear model for power consumption by operating system services. The resultant models are a function of only IPC. Their modeling only considers operating system routines and requires a separate model for each operating system routine. Most importantly, their model is simulation-based and consequently does not correlate well with power consumption of actual processors. In contrast, the models in this dissertation is based on direct, in-system measurement and shows that power depends more on

fetched $\mu$ op/cycle rather than IPC. Bellosa [Be00] uses synthetic workloads to 
demonstrate a correlation between observable performance events and power consumption. He shows that a correlation exists for: $\mu$ ops/sec, f $\mu$ ops/sec, L2 accesses/sec and memory accesses/sec. Since only synthetic workloads are characterized, these results are not representative of realistic workloads. The most closely related work is by Isci et al [IsMa03]. They build a comprehensive power model based on utilization factors of the various components of the processor. Using 22 performance monitoring counters they model average power consumption of SPEC2000 workloads within 5\%. The models in this dissertation yields similar accuracy, yet with only two PMC metrics that aggregate power consumption across the numerous processor functional units. A major limitation of all of these contemporary works is the lack of awareness of power management and temperature effects. The dissertation models accurately account for power fluctuations due to clock gating, DVFS and temperature variation.

Existing studies in system-level power modeling [Ja01] [ZeSo03] [KiSu06] have relied on events local to the subsystem. The model in this dissertation is the first to encompass complete system power using only events local to the processor. The most closely related work by Heath [GuSi02], models CPU, network and disk power using operating system counters. This model does not account for memory and chipset power. Since it relies on comparatively high-overhead operating system routines, the run-time performance cost is higher compared to the dissertation model that uses only fast, on-chip performance counters. 


\subsection{System-Level Power Characterization}

Existing workload power studies of computing systems consider the various levels: microarchitecture [IsMa03] [Be00] [NaHa03], subsystem [BoEl02] [MaVa04] [FeGe051], or complete system [ChAn01]. This disseration targets the subsystem level and extends previous studies by considering a larger number of subsystems. Unlike existing subsystem studies that analyze power on desktop or mobile uniprocessor systems, this dissertation considers multi-core, multi-socket, desktop, mobile and server systems.

Studies at the microarchitecture level [IsMa03] [Be00] utilize performance monitoring counters to estimate the contribution to microprocessor power consumption due to the various functional units. These studies only consider uniprocessor power consumption and use scientific workloads only. Since power is measured through a proxy it is not as accurate as direct measurement. Natarajan [ $\mathrm{NaHa03}$ ] performs simulation to analyze power consumption of scientific workloads at the functional unit level.

At the subsystem level, [BoEl02] [MaVa04] [FeGe05-1] consider power consumption in three different hardware environments. Bohrer [BoEl02] considers CPU, hard disk, and combined memory and $\mathrm{I} / \mathrm{O}$ in a uniprocessor personal computer. The workloads represent typical webserver functions such as http, financial, and proxy servicing. This disseration adds multiprocessors, and considers memory and I/O separately. Mahesri and 
Vardan [MaVa04] perform a subsystem level power study of a Pentium M laptop. They present average power results for productivity workloads. In contrast, this dissertation considers a server-class SMP running a commercial workload. Feng [FeGe05-1] performs a study on a large clustered system running a scientific workload. As part of a proposed resource management architecture, Chase [ChAn01] presents power behavior at the system level. Lacking in all of these studies is a consideration of power phase duration. Duration is a critical aspect since it directs power adaptions. An effective adaptation scheme must choose adaptations that are appropriate to the expected duration of the event. For example, since there is a performance and energy cost associated with DVFS, changes to voltage/frequency should only be performed if the system can amortize those costs before the next change is required.

\subsection{Predictive Power Adaptation}

While most existing power management schemes are reactive, there are a few related proposals that use predictive power management [IsBu06] [DuCa03] [DiSo08]. Isci [IsBu06] uses table-based predictors of memory operations/instruction, to direct DVFS decisions for single-threaded workloads. Duesterwald et al. [DuCa03] examine tablebased predictor techniques to predict performance-related metrics (IPC, cache misses/instruction and branch misprediction rates) of single-thread workloads, but not

power. Diao [DiSo08] uses machine learning to predict activity patterns. The predictions are used to make policy decisions for entering core idle states. In contrast the 
prediction scheme in this dissertation makes use of table-based prediction structures and the repetitive nature of power phases to predict performance demand and/or power consumption. Further, the validation of the predictor is performed using realistic, representative workloads. These workloads contain complex power management events that are not present in the simple workloads used in the contemporary research. These events are critical to a practical power management schemed since they induce power and performance effects larger than those seen in simple workloads.

Outside the predictive adaptation realm, there are numerous proposals for increasing energy efficiency and staying within operating limits. To increase energy efficiency studies have applied adaptation at the processor level [LiMa06] [LiBr05] [WuJu05] [Vi07] [PaSt06] [KoGh05] [KoDe04] and system level [MeGo09] [RaLe03] [BoEl02]. To stay within defined operating limits studies have applied adaptation at the processor level [HaKe07] [RaHa06] [ChJa09] [IsBu06] [McPo06] and complete system level [LeWa07] [RaLe06] [ChDa05] [ChAn01] [Po10] [FaWe07] [WaCh08] [MiFr02].

\subsection{Deadline and User-Driven Power Adaptation}

In the embedded and real-time computing domains power management is performed under a different set of requirements. Rather than focusing on reduction of average or peak power, computation deadlines are more critical. These systems contain various components and processes with critical deadlines for computation. For example, network devices implement buffers to allow the network interface to queue transactions. This 
frees the main processor to service other subsystems in an uninterrupted manner. However, since the buffering is finite, the main processor must service and empty the buffer with the deadline or connectivity may be lost. To reduce energy consumption, several researchers [LuCh00] [KrLe00] [OkIs99] [PeBu00] [ShCh99] [PiSh01] [PoLa01] propose operating the processor at the minimum voltage and frequency that satisfies the computation deadlines as indicated by the kernel or application software. The limitation of this approach is that the deadline must be articulated in the kernel or application software. Other approaches infer deadlines through measurement and/or classification [GoCh95] [PeBu98] [WeWe94] [FlRe01] [LoSm01]. Measurement-based feedback can also be done using not-traditional metrics. Shye [ShSc08] [ShOz08] shows that user satisfaction can be correlated to performance monitoring counters and biometric sensors. Using these metrics, processor performance can be adjusted to the minimum level that satisfies the user. 


\section{Chapter 9 Conclusions and Future Work}

\subsection{Conclusions}

The widespread application of dynamic power management has provided the opportunity for computing systems to attain high performance and energy efficiency across a wide range of workloads. Practically, systems do not operate optimally due to the lack of effective, power management, control schemes. This is largely due to a lack of run-time power accounting and the use of reactive power management on workloads with widely varying performance and power characteristics. The objective of this dissertation is to improve the effectiveness of dynamic power management by addressing these limitations. This is achieved in the following contributions:

\section{1) Fine-Grain Accounting of Complete System Power Consumption}

Using a small set of widely available performance events including IPC, cache misses and interrupts, power models are developed by measuring power consumption on actual systems. These linear and polynomial models are created using regression techniques that iteratively adjust coefficients to minimize model error. This novel approach improves upon existing research by finding that complex structures such as processors and chipsets can be accurately represented by tracking their dominant performance events. Unlike existing research that primarily relies on retired instruction or 
comprehensive performance events, this approach relies on speculative events. This approach provides higher accuracy with many fewer events to track. For a state-of-theart multi-core processor, these simple models achieve average error rates less than $1 \%$.

Through an analysis of power and performance for complete systems it is discovered that the performance events local to the processor can also predict power consumption in the complete system. This trickle-down concept is applied to allow accurate modeling of memory controllers, system memory, caches, chipsets, disks and I/O bridges using only performance events in the cpu. This reduces complexity and measurement overhead by containing all run-time measurement in low-latency on-chip performance counters.

\section{2) Power and Performance Analysis of Computing Systems}

To inform the development of power accounting and management schemes, this dissertation presents an extensive analysis of power and performance of server, desktop and mobile systems. It is found that the largest variation in power and performance occurs in processors and the subsystems most closely coupled to them, such as caches and memory controllers. The cause is largely dictated by the application of power saving techniques such as clock gating and dynamic voltage and frequency scaling. Compared to power variations due to instruction or transactions streams, variation due to power management is much larger.

Additionally, this dissertation presents a new way of analyzing power consumption. Unlike existing research that focuses on average power consumption, this study considers 
the duration of power and performance phases. All power management schemes incur an energy and performance penalty when the system transitions from one adaptation level to another. To avoid costly transitions, adaption schemes must know how and when to adapt. This new approach leads to the discovery of frequently repeating power and performance patterns within workloads. Of these patterns, the most dominant and predictable is the scheduling quanta of operating systems. Since active-idle and idleactive transitions frequently occur on these boundaries, they serve as strong indicators of phase changes.

\section{3) Predictive Power Management}

This dissertation presents the concept of core-level phase prediction and its application to dynamic power management. By observing changes in performance demand and power consumption at the core-level, it is possible to perceive predictable phase behavior. Prediction of phases allows power management to avoid over or under provisioning resources in response to workload changes. Using this concept the PPPP is developed. It is a simple, table-based prediction scheme for directing DVFS selection. The predictor is applied to the SYSmark2007 benchmark suite and achieves significant performance and power improvements. Compared to the reactive DVFS algorithm used by Windows Vista, performance is increased by $5.4 \%$ and while power consumption is reduced by $3.8 \%$. 


\subsection{Future Work}

Run-time energy accounting and predictive power management are promising tools for improving the energy efficiency and performance of computing systems. This dissertation has demonstrated the initial implementation of these tools at improving processor efficiency and performance. Listed below are a few similar research areas that are likely to yield new, valuable discoveries.

\section{Power Managing Cloud Computing Resources}

The shift away from desktop computing to cloud computing is increasing the opportunity for predictive power management. A typical desktop system has about sixty active processes, with less than ten of them actively consuming most computing resources. In contrast, cloud computing servers combine hundreds of active processes from many clients. Each of the processes has phase behavior that is independent of the others. This combination of heterogeneous tasks makes existing reactive power management difficult since the active-idle patterns are an aggregation of multiple independent patterns. A significant power savings opportunity exists in accounting for and predicting the effective usage pattern of cloud computing servers.

\section{Functional Unit Activity Prediction}

The need to increase power efficiency is pushing microarchitectural power management beyond the core-level to individual functional units. To save power during program 
execution, portions of pipelines or functional units can be effectively resized through clock gating and power gating when not needed. The challenge is to apply these power saving adaptations only when the transition cost can be amortized by long idle phases. By defining prediction metrics for each functional unit, it is possible to detect and predict the critical long-duration idle phases.

\section{Process-Level Power Accounting}

The entirety of this dissertation and other performance-counter power modeling research focuses on attributing power to a particular hardware thread or core. The limitation of this approach is that process scheduling and migration can impact the ability to discern unique program phases. It is likely that tracking power and phase history at the processlevel will reduce aliasing thus improving predictability.

\section{Scheduling using On-line Power and Performance Models}

Another application of power accounting is for the direction of scheduling decisions. By introducing performance models that are power-aware, optimal scheduling and power management decisions can be made. The current state-of-the-art architectural power adaptations provide a range of throughput and latency at the processor core-level. Expressing those impacts as run-time power and performance models would allow major improvements in energy efficiency and performance. 


\section{Bibliography}

[Ac07] Advanced Configuration \& Power Interface. http://www.acpi.info (November 2007).

[Ag08] Agilent Technologies. 1146A $100 \mathrm{kHz} / 100 \mathrm{~A}$ AC/DC Current Probe. http://www.agilent.com, April 2008.

[An73] F. J. Anscombe. Graphs in Statistical Analysis. American Statistician, pages 17-21, February 1973.

[Be00] F. Bellosa. The Benefits of Event-Driven Energy Accounting in PowerSensitive Systems. In Proceedings of the 9th Workshop on ACM SIGOPS European Workshop: Beyond the PC: New Challenges for the Operating System (Kolding, Denmark, September 2000), 37-42.

[Bk09] BIOS and Kernel Developer's Guide for AMD Family 10h Processor. http://www.amd.com. November 2009.

[BiVa05] W. L. Bircher, M. Valluri, J. Law, and L. John. Runtime identification of microprocessor energy saving opportunities. In Proceedings of the 2005 International Symposium on Low Power Electronics and Design (San Diego, California, August 2005), 275-280. 
[BiJo06-1] W. L. Bircher and L. John. Complete System Power Estimation: A Trickle-Down Approach based on Performance Events. In IEEE International Symposium on Performance Analysis of Systems and Software (San Jose, California, April 2007), 158-168.

[Bi06] W. L. Bircher. Measurement Based Power Phase Analysis of a Commercial Workload. $2^{\text {nd }}$ IEEE Workshop on Unique Chips and Systems (Austin, Texas, March 2006).

[BiJo06-3] W. L. Bircher and L. John. Power Phase Availability in a Commercial Server Workload. In Proceedings of the 2006 International Symposium on Low Power Electronics and Design (Tegernsee, Germany, October 2006), 350-353.

[BiJo08] W. L. Bircher and L. John. Analysis of Dynamic Power Management on Multi-Core Processors. In Proceedings of the $22^{\text {nd }}$ Annual International Conference on Supercomputing (Kos, Greece, June 2008), 327-338.

[BiJo10-1] W. L. Bircher and L. John. Complete System Power Estimation using Processor Performance Events. Under Review at IEEE Transactions on Computers (October 2010).

[BiJo10-2] W. L. Bircher and L. John. Predictive Power Management for Multi-Core Processors. In Workshop on Energy Efficient Design (Saint-Malo, France, June 2010), $327-338$. 
[B107] Blade Server Technology Overview. http://www.blade.org/techover.cfm (March 2007).

[BoEl02] P. Bohrer, E. N. Elnozahy, T. Keller, M. Kistler, C. Lefurgy, C. McDowell, and R. Rajamony. The Case For Power Management in Web Servers. IBM Research, Austin TX, 2002.

[BrTiMa00] D. Brooks, V. Tiwari, and M. Martonosi. Wattch: A Framework for Architectural-Level Power Analysis and Optimizations, In Proceedings of the $27^{\text {th }}$ Annual International Symposium on Computer Architecture, (Vancouver, British Columbia, Canada, June 2000), 83-94.

[ChJa09] J. Charles, P. Jassi, N. Ananth, A. Sadat, and A. Fedorova. Evaluation of the Intel® Core ${ }^{\mathrm{TM}} \mathrm{i} 7$ Turbo Boost feature. In Proceedings of the 2009 IEEE International Symposium on Workload Characterization (Austin, Texas, October 2009), 188-197.

[ChAn01] J. Chase, D. Anderson, P. Thakar, and A. Vahdat. Managing Energy and Server Resources in Hosting Centers. In Proceedings of the 18th ACM Symposium on Operating System Principles (Chateau Lake Louise, Banff, Alberta, Canada, October 2001), 103-116.

[ChDa05] Y. Chen, A. Das, W. Qin, A. Sivasubramaniam, Q. Wang, and N. Gautam, Managing Server Energy and Operational Costs in Hosting Centers. In Proceedings of 
the 2005 ACM SIGMETRICS International Conference on Measurement and Modeling of Computer Systems (Banff, Alberta, Canada, June 2005), 303-314.

[CoMa05] G. Contreras and M. Martonosi. Power Prediction for Intel XScale Processors Using Performance Monitoring Unit Events. In Proceedings of the 2005 International Symposium on Low Power Electronics and Design (San Diego, California, August 2005), 221-226.

[DhSm03] A. Dhodapkar and J. Smith. Comparing program phase detection techniques. In Proceedings of the $36^{\text {th }}$ Annual International Symposium on Microarchitecture (San Diego, California, December 2003), 217-228.

[DiSo08] Q. Diao and J. Song. Prediction of CPU Idle-Busy Activity Pattern. In Proceedings of the International Symposium on High-Performance Computer Architecture (Salt Lake City, Utah, February 2008), 27-36.

[DuCa03] E. Duesterwald, C. Cascaval, and S. Dwarkadas. Characterizing and Predicting Program Behavior and its Variability. In Proceedings of the $12^{\text {th }}$ International Conference on Parallel Architectures and Compilation Technique (New Orleans, Louisiana, September 2003), 220-231.

[Po10] Dynamic Power Capping TCO and Best Practices White Paper. http://h20195.www2.hp.com/v2/GetPDF.aspx/4AA2-3107ENW.pdf (May 2010). 
[FaWe07] X. Fan, W. Weber, and L. Barroso. A. Power provisioning for a warehouse-sized computer. In Proceedings of the 34th Annual International Symposium on Computer Architecture (San Diego, California, June 2007), 13-23.

[FeGe05-1] X. Feng, R. Ge, and K. W. Cameron. Power and Energy Profiling of Scientific Applications on Distributed Systems. In Proceedings of the $19^{\text {th }}$ IEEE International Parallel \& Distributed Processing Symposium (Denver, Colorado, April 2005), 34-50.

[GaJo10] K. Genesan, J. Jo, W. L. Bircher, D. Kaseridis, Z. Yu and L. John. System-level Max Power (SYMPO) - A Systematic Approach for Escalating SystemLevel Power Consumption using Synthetic Benchmarks. In Proceedings of the 2010 International Conference on Parallel Architectures and Compilation Techniques (Vienna, Austria, June 2010), 19-28.

[GuSi02] S. Gurumurthi, A. Sivasubramaniam, M. J. Irwin, N. Vijaykrishnan, M. Kandemir, T. Li, and L. K. John. Using Complete Machine Simulation for Software Power Estimation: The SoftWatt Approach. In Proceedings of the 8th International Symposium on High-Performance Computer Architecture (Boston, Massachusetts, February 2002), 141-150.

[HaKe06] H. Hanson and S. W. Keckler. Power and Performance Optimization: A Case Study with the Pentium M Processor. 7th International Austin Center for Advanced Studies (IBM) Conference (Austin, Texas, February 2006). 
[HaKe07] H. Hanson, S.W. Keckler, K. Rajamani, S. Ghiasi, F. Rawson, and J. Rubio. Power, Performance, and Thermal Management for High-Performance Systems. In Proceedings of The Third Workshop on High-Performance, Power-Aware Computing, held in conjunction with 21st Annual International Parallel \& Distributed Processing Symposium (Long Beach, California, March 2007).

[HeCe06] T. Heath, A. P. Centeno, P. George, L. Ramos, Y. Jaluria, and R. Bianchini. Mercury and Freon: Temperature Emulation and Management in Server Systems. In Proceedings of the $12^{\text {th }}$ International Conference on Architectural Support for Programming Languages and Operating Systems (San Jose, California, October 2006), 106-116.

[Am07] Inside Barcelona: AMD's Next Generation http://www.realworldtech.com . November 2007.

[In04] Intel Corporation. IA-32 Architecture Optimization Reference Manual. 2004.

[In06] Intel Software Network. Thermal Protection And Monitoring Features: A Software Perspective. www.intel.com/cd/ids/developer/asmona/eng/newsletter, February 2006. 
[IsMa03] C. Isci and M. Martonosi. Runtime Power Monitoring in High-End Processors: Methodology and Empirical Data. In $36^{\text {th }}$ International Symposium on Microarchitecture (San Diego, California, December 2003), 93-104.

[IsMa06] C. Isci and M. Martonosi. Phase Characterization for Power: Evaluating Control-Flow-Based and Event-Counter-Based Techniques. In Proceedings of the Twelfth International Symposium on High-Performance Computer Architecture (Austin, Texas, February 2006), 122-133.

[IsBu06] C. Isci, A. Buyuktosunoglu, C. Cher, P. Bose, and M. Martonosi. An Analysis of Efficient Multi-Core Global Power Management Policies: Maximizing Performance for a Given Power Budget. In Proceedings of the 39th Annual IEEE/ACM International Symposium on Microarchitecture (Orlando, Florida, December 2006), 347358.

[IsBu06] C. Isci, G. Contreras, and M. Martonosi. Live, Runtime Phase Monitoring and Prediction on Real Systems with Application to Dynamic Power Management. In Proceedings of the $39^{\text {th }}$ Annual IEEE/ACM International Symposium on Microarchitecture (Orlando, Florida, December 2006), 359-370.

[Ja01] J. Janzen. Calculating Memory System Power for DDR SDRAM. Micro Designline, Volume 10, Issue 2, 2001. 
[JoMa01] R. Joseph and M. Martonosi. Runtime Power Estimation in HighPerformance Microprocessors. In Proceedings of the 2001 International Symposium on Low Power Electronics and Design (Huntington Beach, California, 2001), 135-140.

[KiSu06] Y. Kim, S. Gurumurthi, and A. Sivasubramaniam. Understanding the performance-temperature interactions in disk I/O of server workloads. In Proceedings of the Twelfth International Symposium on High-Performance Computer Architecture (Austin, Texas, February 2006), 176- 186.

[KoGh05] R. Kotla, S. Ghiasi, T. Keller, and F. Rawson. Scheduling Processor Voltage and Frequency in Server and Cluster Systems. In Proceedings of the First Workshop on High-Performance Power-Aware Computing in conjunction with International Parallel and Distributed Processing Symposium (Denver, Colorado, April 2005).

[KoDe04] R. Kotla, A. Devgan, S. Ghiasi, T. Keller, and F. Rawson. Characterizing the Impact of Different Memory-Intensity Levels. In Proceedings of the 7th Annual IEEE Workshop on Workload Characterization (Austin, Texas, October 2004), 3-10.

[La10] National Instruments LabVIEW. http://www.ni.com/labview. 2010.

[LaSc04] J. Lau, S. Schoenmackers, and B. Calder. Structures for Phase Classification. In Proceedings of the 2004 IEEE International Symposium on Performance Analysis of Systems and Software (Austin, Texas, March 2004), 57-67. 
[P108] Report to Congress on Server and Data Center Energy Efficiency: Public law 109-431, Lawrence Berkeley National Laboratory, 2008.

[LeSk05] K. Lee and K. Skadron. Using Performance Counters for Runtime Temperature Sensing in High-Performance Processors. In Proceedings of the First Workshop on High-Performance Power-Aware Computing in conjunction with International Parallel and Distributed Processing Symposium (Denver, Colorado, April 2005).

[LeWa10] J. Lee, E. Wang, H. Ghasemi, W. L. Bircher, Y. Cao and N. Kim. Workload-Adaptive Process Tuning Strategy for Power-Efficient Multi-Core Processors. In Proceedings of the 2010 International Symposium on Low Power Electronics and Design (Austin, Texas, August 2010), 225-230.

[LeWa07] C. Lefurgy, X. Wang, and M. Ware. Server-level power control. In Proceedings of the $4^{\text {th }}$ IEEE International Conference on Autonomic Computing (Jacksonville, Florida, June 2007), 4.

[LiMa06] J. Li and J. Martinez. Dynamic Power-Performance Adaptation of Parallel Computation on Chip Multiprocessors. In Proceedings of the 12th International Symposium on High-Performance Computer Architecture (Austin, Texas, February 2006), 77-87. 
[LiBr05] Y. Li, D. Brooks, Z. Hu, and K. Skadron. Performance, Energy, and Thermal Considerations for SMT and CMP Architectures. In Proceedings of the $11^{\text {th }}$ International Symposium on High-Performance Computer Architecture (San Francisco, California, February 2005), 71-82.

[LiJo03] T. Li and L. John. Run-Time Modeling and Estimation of Operating System Power Consumption. In Proceedings of the 2003 ACM SIGMETRICS International Conference on Measurement and Modeling of Computer Systems (San Diego, California, June 2003), 160-171.

[Pe06] Linux Perfctr Kernel Patch Version 2.6, user.it.uu.se/ mikpe/linux/perfctr, October 2006.

[MeGo09] D. Meisner, B. Gold, and T. Wenisch. PowerNap: Eliminating Server Idle Power. In Proceedings of the $14^{\text {th }}$ International Conference on Architectural Support for Programming Languages and Operating Systems (Washington, DC, March 2009), 205216.

[MaDo09] X. Ma, M. Dong, L. Zhong, and Z. Deng. Statistical Power Consumption Analysis and Modeling for GPU-based Computing. In Proceedings of the ACM SOSP Workshop on Power Aware Computing and Systems (HotPower) 2009, (Big Sky, Montana, October 2009). 
[MaVa04] A. Mahesri and V. Vardhan. Power Consumption Breakdown on a Modern Laptop, In Proceedings of the $4^{\text {th }}$ International Workshop on Power-Aware Computing Systems (Portland, Oregon, December 2004), 165-180.

[McPo06] R. McGowen, C. Poirier, C. Bostak, J. Ignowski, M. Millican, W. Parks, and S. Naffziger. Temperature Control on a 90-nm Itanium Family Processor. IEEE Journal of Solid State Circuits, Vol. 41, No. 1, January 2006.

[Mc04] Conversation with Gregg McKnight, IBM Distinguished Engineer, xSeries Division. September 2004.

[MiFr02] R. J. Minerick, V. W. Freeh, and P. M. Kogge. Dynamic Power Management Using Feedback. In Proceedings of the $3^{\text {rd }}$ Workshop on Compilers and Operating Systems for Low Power (COLP'02) (Charlottesville, Virginia, September 2002).

[NaHa03] K. Natarajan, H. Hanson, S. Keckler, C. Moore, and D. Burger. Microprocessor Pipeline Energy Analysis, In Proceedings of the 2003 IEEE International Symposium on Low Power Electronics and Design (Seoul, Korea, August 2003), 282287.

[Ni08] National Instruments Data Acquisition Hardware. http://www.ni.com/dataacquisition/ (April 2008). 
[Os06] Open Source Development Lab, Database Test 2, www.osdl.org, February 2006.

[PaSt06] V. Pallipadi and A. Starikovskiy. The On Demand Governor: Past, Present and Future. In Proceedings of the Linux Symposium (Ottawa, Canada, July 2006), 223238.

[PS06] PostgreSQL, www.postgresql.org, October 2006.

[Vi07] Processor Power Management in Windows Vista and Windows Server 2008. http://www.microsoft.com (November 2007).

[RaHa06] K. Rajamani, H. Hanson, J. Rubio, S. Ghiasi, and F. Rawson. ApplicationAware Power Management. In Proceedings of the 2006 IEEE International Symposium on Workload Characterization (San Jose, California, October 2006), 39-48.

[RaLe03] K. Rajamani and C. Lefurgy. On Evaluating Request- Distribution Schemes for Saving Energy in Server Clusters. In Proceedings of the 2003 IEEE International Symposium on Performance Analysis of Systems and Software (Austin, Texas, March 2003), 111-122.

[RaIb07] K. Ramani, A. Ibrahim, and D. Shimizu. PowerRed: Flexible Modeling Framework for Power Efficiency Exploration in GPUs. In Proceedings of the First Worskshop on General Purpose Processing on Graphics Processing Units (GPGPU) (Boston, Massachusetts, October 2007). 
[RaLe06] P. Ranganathan, P. Leech, D. Irwin, and J. Chase. Ensemble-Level Power Management for Dense Blade Servers. In Proceedings of the $33^{\text {rd }}$ International Symposium on Computer Architecture (Boston, Massachusetts, June 2006), 66-77.

[SiPa07] S. Siddah, V. Pallipadi, and A. Van de Ven. Getting Maximum Mileage Out of Tickless. In Proceedings of the $9^{\text {th }}$ Linux Symposium (Ottawa, Canada, June 2007), 201-207.

[Sp00] SPEC CPU 2000 Version 1.3, www.spec.org/cpu2000, October 2006.

[Sp06] SPEC CPU 2006 Version 1.1, www.spec.org/cpu2006, October 2006.

[Sj06] SPECjbb 2005 Version 1.07, www.spec.org/jbb2005, October 2006.

[Sp02] B. Sprunt. Pentium 4 Performance Monitoring Features, Micro, JulyAugust, pp 72-82, 2002.

[Se06] Texas Instruments. INA168 High-Side Measurement Current Shunt Monitor. ti.com, May 2006.

[Sm07] Business Applications Performance Corporation. SYSmark 2007 an Overview of SYSmark 2007 Preview, May 2008.

[3d06] Futuremark. 3DMark06 Whitepaper v1.02, January 2006. 
[WaCh08] X. Wang and M. Chen. Cluster Level Feedback Power Control for Performance Optimization. In Proceedings of the $14^{\text {th }}$ International Symposium on HighPerformance Computer Architecture (Salt Lake City, Utah, February 2008), 101-110.

[Mm05] Windows Multimedia: timeEndPeriod(). http://msdn.microsoft.com/enus/library/ms713415(VS.85).aspx (November 2008).

[WuJu05] Q. Wu, P. Juang, M. Martonosi, L. Peh, and D. Clark. Formal control techniques for power-performance management. IEEE Micro, 25, 5 (September/October 2005).

[ZeSo03] J. Zedlewski, S. Sobti, N. Garg, F. Zheng, A. Krishnamurthyy, and R. Wang. Modeling Hard-Disk Power Consumption. File and Storage Technologies 2003.

[ShSc08] A. Shye, Y. Pan, B. Scholbrock, J.S. Miller, G. Memik, P.A. Dinda and R.P. Dick. Power to the People: Leveraging Human Physiological Traits to Control Microprocessor Frequency Learning and Leveraging the Relationship between Architecture-Level Measurements and Individual User Satisfaction. In Proceedings of 41st IEEE/ACM International Symposium on Microarchitecture, (Lake Como, Italy, November 2008), 188-199.

[ShOz08] A. Shye, B. Ozisikyilmaz, A. Mallik, G. Memik , P.A. Dinda , R.P. Dick and A.N. Choudhary. Learning and Leveraging the Relationship between ArchitectureLevel Measurements and Individual User Satisfaction. In Proceedings of the 35th 
International Symposium on Computer Architecture, (Beijing, China, June 2008), 427438.

[LoSm01] J. Lorch and A.J. Smith. Improving dynamic voltage scaling algorithms with PACE. In Proceedings of the 2001 ACM SIGMETRICS International Conference on Measurement and Modeling of Computer Systems (Cambridge, Massachusetts, June 2001), 50-61.

[KrLe00] C.M. Krishna and Y-H Lee. Voltage-Clock-Scaling Adaptive Scheduling Techniques for Low Power Hard Real-Time Systems. In Proceedings of the $6^{\text {th }}$ IEEE RealTime Technology and Applications Symposium (Washington, District of Columbia, May 2000), 1586 - 1593.

[OkIs99] T. Okuma, T. Ishihara, and H. Yasuura. Real-Time Task Scheduling for a Variable Voltage Processor. In Proceedings of the $12^{\text {th }}$ International Symposium on System Synthesis (San Jose, California, November 1999), 87-94.

[PeBu00] T. Pering, T. Burd, and R. Brodersen. Voltage Scheduling in the lpARM Microprocessor System. In Proceedings of the International Symposium on Low Power Electronics and Design (Vancouver, British Columbia, June 2000), 96-101.

[ShCh99] Y. Shin and K. Choit. Power Conscious Fixed Priority Scheduling for Hard Real-Time Systems. In Proceedings of the $36^{\text {th }}$ Annual Design Automation Conference (Atlanta, Georgia, October 1999), 134-139. 
[PiSh01] P. Pillai and K. G. Shin. Real-time Dynamic Voltage Scaling for LowPower Embedded Operating Systems. In Proceedings of the 18th Symposium on Operating System Principles (Chateau Lake Louise, Banff, October 2001),89-102.

[PoLa01] J. Pouwelse, K. Langendoen, and H. Sips. Voltage scaling on a low-power microprocessor. In Proceedings of the $7^{\text {th }}$ International Conference on Mobile Computing and Networking (Rome, Italy, July 2001), 251-259.

[GoCh95] K. Govil, E. Chan, and H. Wasserman. Comparing Algorithms for Dynamic Speed-Setting of a Low-Power CPU. In Proceedings of the First International Conference on Mobile Computing and Networking (Berkeley, California, November 1995), 13-25.

[PeBu98] T. Pering, T. Burd, and R. Brodersen. The Simulation and Evaluation of Dynamic Voltage Scaling Algorithms. In Proceedings of International Symposium on Low Power Electronics and Design (Monterey, California, June 1998), 76-81.

[WeWe94] M. Weiser, B. Welch, A. Demers, and S. Shenker. Scheduling for Reduced CPU Energy. In Proceedings of the First Symposium of Operating Systems Design and Implementation (Monterey, California, November 1994), 13-23.

[FlRe01] K. Flautner, S. Reinhardt, and T. Mudge. Automatic Performance-Setting for Dynamic Voltage Scaling. In Proceedings of the $7^{\text {th }}$ International Conference on Mobile Computing and Networking (Rome, Italy, July 2001), 260-271. 


\section{Vita}

William Lloyd Bircher was born in Wichita, Kansas on December $14^{\text {th }}, 1973$ to Mr. William Mark Bircher and Catherine Ann Bircher. After living in Kansas for thirteen years, he moved to Fort Worth, Texas with his family. In the Fall of 1992 he began his undergraduate studies in the College of Engineering at the University of Texas at Arlington. He received a Bachelor of Science in Electrical Engineering in 1997. After graduation he joined Compaq Computer Corporation developing system firmware code. In 2000 he joined International Business Machines also as a firmware code developer. That same year he enrolled at the University of Texas at Austin to begin graduate studies in computer engineering as a part-time student. In 2004 he married Sara Marie Smith. In May 2006 he received a Master of Science degree in Electrical and Computer Engineering. Lloyd's graduate education was supported by International Business Machines and Advanced Micro Devices.

Email Address: 1loydbircher@gmail.com

This manuscript was typed by William Lloyd Bircher. 